system and derive the moment-generating function (MGF) over Rayleigh fading links. In [10], the authors examined the relay selection-based, full-duplex AF relaying protocol considering a scenario in which the primary users (PUs) have access to spectrum and many pairs of secondary users (SUs) have no dedicated spectrum. Firstly, the authors derived a mathematical framework to maximize the rate of $\mathrm{PU}$ by optimizing the transmission power in the relay while keeping the SU rate stable. Secondly, lowcomplexity relay selection schemes have been proposed based on the maximum rate, i.e., the priority matching and centralized gradual schemes. In [11], the authors consider a piecewise linear detector (PLD) and investigate the SER performance of three-node one-way DF cooperative communication over Rayleigh fading channel conditions. From this work, authors consider the perfect CSI and obtained the suboptimal error performance. In [12], the authors had employed the maximum ratio transmission (MRT) technique to investigate the SER and OP performance of a two-way DF relaying network over generalized fading channel conditions. It had been demonstrated that for SER performance improves with MRT scheme. However, in the works [8-12], noise amplification and erroneous signal propagation are two main drawbacks and to mitigate this problem S-DF protocol-based relaying networks proposed in the works $[13,14]$.

In the work [13], the symbol error rate (SER) expression of the S-DF cooperative communication network is derived considering keyhole or pinhole channel conditions. It is observed that due to keyhole condition the SER performance degrades. In [14], the authors derive the OP expression of DH S-DF relaying network in terms of hypergeometric function of two variables considering perfect CSI over Nakagami- $m$ fading channel conditions. However, the works [13, 14] did not, however, consider real-time propagation constraints such as node mobility and channel estimation errors. The authors investigate the AF relaying network in the works [15-17] over time-selective fading links considering high node's mobility and imperfect CSI. In paper [15], the authors investigate the AF relaying network considering conventional and best relay selection relaying schemes over time-selective Rayleigh fading links considering imperfect CSI and node mobility. But in this work, the author did not investigate the Shannon capacity and OP. In [16], the authors investigate the OP performance of the multiple relay AF relaying network over timeselective Rayleigh fading links considering imperfect CSI and node mobility. In [17], the authors consider the channel state information and noise (CSIN)-assisted AF relaying network in time-selective Rayleigh fading conditions with imperfect CSI and high node's mobility and derive generic, a novel, and tractable exact expression for per-block average SER. Again, the main drawback of the works [15-17] is the issue of noise amplification occurs because of the AF protocol. To overcome this problem, authors in the works [18-20] consider S-DF relaying protocol over time-selective Rayleigh fading conditions.

In [18], the authors investigate the symbol error rate (SER) performance of the hybrid satellite terrestrial relaying network (HSTRN) over time-selective Rayleigh fading links considering node mobility and imperfect CSI. However, in this paper, the authors did not investigate the OP performance. In [19], the authors derive the CF expressions of OP and PEP for multiple-input multiple-output (MIMO) S-DF relaying network over time-selective Rayleigh fading channel conditions with imperfect CSI and node mobility. It has been observed that the OP and PEP performance improves for optimal power factors. In [20], the authors investigated the MIMO space-time block code (STBC)based S-DF relaying network considering node mobility and incorrect CSI. The authors derived a mathematical framework for obtaining the optimal source-relay power allocation factors. CF expressions of per-block average PEP are derived for optimal power allocation and various node mobility scenarios. But in this work authors consider only phase-shift keying (PSK)-modulated symbols and analysis are missing for quadrature amplitude modulation (QAM) and frequency shift keying (FSK)-modulated symbols. The main drawback of the works [15-20] is that these works only consider the time-selective Rayleigh's fading links and did not consider the generalized fading channel distribution.

The Rayleigh channel model only gives the DO of one, and it does not consider the line-of-sight (LOS) transmission environments. Nakagami- $m$ fading model [21-23] is a generalized distribution that can model different fading environments and provide DO equal to $m$. It has greater flexibility and accuracy in matching some experimental data than the Rayleigh, lognormal, or Rice distributions. Rayleigh and one-sided Gaussian distribution are special cases of the Nakagami- $m$ model. Nakagami- $m$ channel model is of more general applicability in practical fading channels. Also, the mathematical form of Nakagami-m model is more analytical tractable. In the works [24-27], a cooperative communication network is investigated over the generalized fading channel model. In [24], the authors investigate the impact of latency on full-duplex AF relaying network over the Nakagami- $m$ fading channel distribution. In this work, the direct link between the source and destination is considered, and at the destination node, the optimal combining scheme is used to combine the signals received from the source and relay nodes.

In [25], the authors investigate derive the exact OP expression for the full-duplex relaying network over Nakagami- $m$ fading channels. In this work, the authors investigate the impact of the number of antennas, residual 
self-interference, and the time interval of harvesting. In [27], the authors investigate the secrecy OP performance of a DH cooperative communication network over Nakagami- $m$ fading channel conditions. In this work, the authors investigate whether the non-cooperative link between two communication nodes is not suitable to achieve the desired OP performance, due to the presence of heavy shadows and severe fading. Therefore, one relay is opportunistically selected among $K$ relay nodes to establish their communications. But in the works [24-27], the authors did not consider the real-time propagation constraints such as time selectivity and imperfect CSI. The time-selective Nakagami- $m$ fading channel model best captures wireless channels for mobile nodes and has been well investigated for machine-to-machine (M2M) [28] and device-to-device [29] wireless communication scenarios and experiential models based on Nakagami- $m$ fading channel. Besides, the Nakagami- $m$ fading channel model is also valid for practical scenarios where nodes are far apart from each other. In this work, the time difference of the wireless streaming channel is captured by the first-order autoregressive (AR1) channel model [30, 31]. Thus, to examine the end-to-end error performance of cooperative vehicular networking [32-35], the time-selective Nakagami- $m$ fading channel is a standard model considering the node mobility and imperfect CSI estimation.

In this paper, we investigate the DH- and MH S-DFbased relaying network over time-selective Nakagami- $m$ fading channel conditions considering node mobility and imperfect CSI. The analysis is generalized, and it applies to various types of modulation schemes.

Notation The following notations are used in the mathematical expressions presented in this paper. $\|X\|_{F}, F_{A}^{(1)}\left({ }_{i}, ; i,\right)$ represent the Euclidean norm and Appell hypergeometric function of two variables, respectively, $X^{H},|X|$ and $\operatorname{trace}(X)$ represent the conjugate transpose, absolute value, and trace of the matrix $X$, respectively. $\Phi_{1}\{a, b, c, x, y\}$ represents the confluent hypergeometric function of two variables $x$ and $y_{r_{x}} F_{y}(\ldots, \ldots)$ represents the Gauss hypergeometric function. $E\{$.$\} represents the expectation operator, and$ $J_{0}\{$.$\} represents the Bessel function of the first kind and$ zeroth order. $\Gamma($.$) and \gamma($, ) represent the Gamma function and lower incomplete Gamma function, respectively. $P(Z>a)$ represents the probability that a standard normal random variable $Z$ is greater than a given value $a$.

The rest of the paper is organized as follows: In Sect. 2, we will discuss the channel fading and a signal model of the DH and MH S-DF cooperation protocol. In Sect. 3, we derive the per-block average PEP, OP, asymptotic error floor, and asymptotic PEP expressions for DH protocol over time-selective Nakagami- $m$ fading channel considering node mobility and imperfect CSI. In Sect. 4, a similar analysis is given to the $\mathrm{MH}$ multiple relay protocol. Convex optimization (CO) framework is developed for finding the DO and optimal source-relay power allocation factors. In Sect. 5, simulation results are given, and finally, in Sect. 6, we have concluded the paper.

\section{System model}

We consider a cooperative communication system with $L$ number of relays. Relay nodes are ready to cooperate with the source node in forwarding its signal to the destination node over orthogonal channels, which could be attained either by code division multiple access (CDMA), frequency division multiple access (FDMA), or time division multiple access (TDMA). Destination, relay, and source nodes are equipped with $M_{D}, M_{R}$ and $M_{S}$ number of antennas, respectively. To keep the information rate of the source-to-bth relay fading link the same as that of the $b$ th relay-to-destination fading link, we herein propose to use the same OSTBC at the source and relay, respectively. This also means that $M_{\mathrm{S}}=M_{\mathrm{R}}=M$. OSTBC code which makes full use of the transmit diversity in the configuration $2 T_{X}-1 T_{X}[36]$ is chosen in $802.11 \mathrm{n}$ standard as an option. In OSTBC, $k_{1}$ symbols are transmitted in $k_{2}$ times intervals; therefore, the OSTBC code rate is $\tilde{R}=k_{1} / k_{2}$ symbols/sec. Let $B_{s}(t) \in \mathbb{C}^{M \times T_{s}}$ represent the Alamouti OSTBC code word and $\Upsilon \in\left\{B_{s}(t)\right\} ; 1 \leq s \leq|\Upsilon|$, represent the Alamouti OSTBC code word set where $|Y|$ represents the cardinality of the set $\Upsilon, t$ represents the $t$ th OSTBC code word in a frame of $M_{b}$ OSTBC code word's [37] and $T_{\mathrm{S}}$ represents the block length. In this relaying scheme, signal transmission takes place in two phases; in the first phase called the broadcast phase, the source transmits Alamouti OSTBC code word $B_{s}(t)$ to all relays and destination, respectively. In the second phase called the relaying phase, $b$ th relay forwards only correctly decode signal to the destination node; otherwise, it will remain in the idle position [38]. In the S-DF relaying scheme, relays perform the decoding operation by setting some threshold SNR. If the received instantaneous SNR at the $b$ th relay is greater than the threshold SNR, then, only, in this case, $b$ th relay will forward the signal to the destination node. In Fig. 1, schematic representation of the basic OSTBC encoder is shown along with the direct and the cooperative links for single relay case, and in Fig. 2 schematic representation of Virtual $(L+1) \times 1$ MIMO system is shown considering $L$ number of relay nodes.

The received code word matrices $R_{\mathrm{SR}}^{(b)}(t) \in \mathbb{C}^{M \times T_{\mathrm{s}}}$ and $R_{\mathrm{SD}}(t) \in \mathbb{C}^{M_{\mathrm{D}} \times T_{\mathrm{S}}}$ at the $b$ th relay and destination nodes, respectively, can be expressed below [39],

$R_{\mathrm{SR}}^{(b)}(t)=\sqrt{P_{\mathrm{S}} \mathbf{c}_{2} / M R_{\mathrm{C}}} A_{\mathrm{SR}}^{(b)}(t) B_{\mathrm{S}}(t)+\mathbb{N}_{\mathrm{SR}}^{(b)}(t) ; \quad 1 \leq b \leq L$, 
Fig. 1 Schematic representation of relaying channel with OSTBC for cooperative wireless system

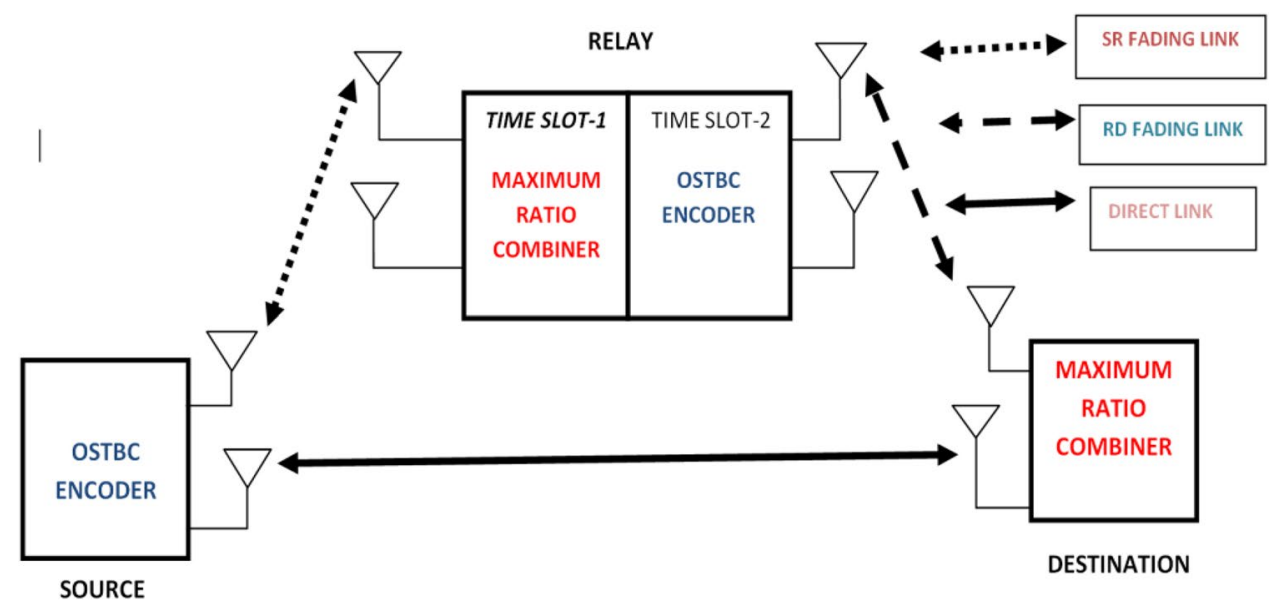

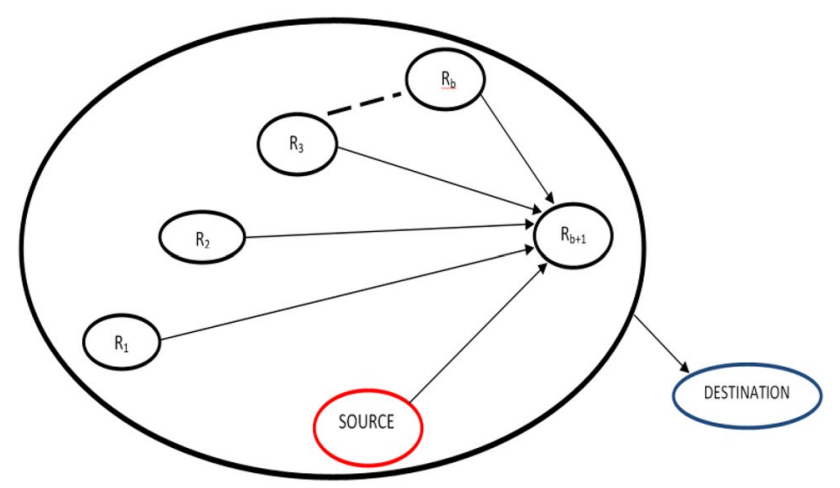

Fig. 2 Schematic representation of cooperative communication system under $W(L-1)$ : the $(b+1)$ th relay optimally combines the signals received from all the previous relay nodes and source node

$R_{\mathrm{SD}}(t)=\sqrt{P_{\mathrm{S}} \mathbf{c}_{0} / M R_{\mathrm{C}}} A_{\mathrm{SD}}(t) B_{\mathrm{S}}(t)+\mathbb{N}_{\mathrm{SD}}(t)$.

where $\mathbf{c}_{2}$ and $\mathbf{c}_{0}$ denote the distance-dependent PTFs for source-to-bth relay and source-to-destination (SD) fading links, respectively, $P_{\mathrm{S}}$ and $R_{\mathrm{C}}$ denote the available power at the source node and coding rate, respectively. The terms of the $S D A_{S D}(t) \in \mathbb{C}^{M_{D} \times M}$ and source-to- $b$ th relay $A_{\mathrm{SR}}^{(b)}(t) \in \mathbb{C}^{M \times M}$ channel matrices are independent with each other, distributed as Nakagami- $m$ RVs with a fading severity or shape parameter, $m_{i} \geq 0.50 ; i \in\{\mathrm{SD}, \mathrm{SR}\}$ and controlling spreads $\sigma_{\mathrm{SD}}^{2}$ and $\left(\sigma_{\mathrm{SR}}^{(b)}\right)^{2}$, respectively. $\mathbb{N}_{S R}^{(b)}(t) \in \mathbb{C}^{M \times T_{S}}$ and $\mathbb{N}_{S D}(t) \in \mathbb{C}^{M_{D} \times T_{S}}$ denote the channel noise matrices for the source-to-bth relay and SD fading links, respectively. These matrices consist of white noise samples, modeled as zero-mean circular shift complex Gaussian (ZMCSCG) random variables (RVs), i.e., $\mathbf{C N}\left(0, N_{0}\right)$. In the broadcast phase, the received code word matrix $R_{\mathrm{RD}}^{(b)}(t) \in \mathbb{C}^{M_{\mathrm{D}} \times T_{\mathrm{s}}}$ at the destination can be expressed as [39],
$R_{\mathrm{RD}}^{(b)}(t)=\sqrt{P_{b} \mathbf{c}_{1} / M R_{\mathrm{C}}} A_{\mathrm{RD}}^{(b)}(t) B_{\mathrm{S}}(t)+\mathbb{N}_{\mathrm{RD}}^{(b)}(t)$.

where $\mathbf{c}_{1}$ denote the distance-dependent PTF for the $b$ th relay-to-destination fading link, $P_{b}$ denote the available power at the $b$ th relay. The terms of the $b$ th relay-to-destination channel matrix $A_{\mathrm{RD}}^{(b)} \in \mathbb{C}^{M_{\mathrm{D}} \times M}$ are independent with each other, distributed as Nakagami- $m$ RVs with a fading severity or shape parameter, $m_{\mathrm{RD}} \geq 0.50$ and controlling $\operatorname{spread}\left(\sigma_{\mathrm{RD}}^{(b)}\right)^{2}$, respectively. $\mathbb{N}_{\mathrm{RD}}^{(b)}(t) \in \mathbb{C}^{M_{\mathrm{D}} \times T_{s}}$ represents the channel noise matrix between $b$ th relay and destination fading link, consists of white noise samples, and modeled as ZMCSCG RVs, i.e., $\mathbf{C N}\left(0, N_{0}\right)$. Let $h_{r, d}(t)$ and $h_{s, r}(t)$ denote the instantaneous channel gains for $b$ th relay transmit antenna to destination's receive antenna and sth source transmit antenna $\left\{s \in\left(1,2,3, \ldots, M_{s}\right)\right\}$ to bth relay received antenna, respectively. Also, the instantaneous channel gain for the fading links from the sth source transmit antenna to destination receive antenna is represented by $h_{s, d}(t)$. In this work, due to nodes' mobility, fading links between SD, source-to-bth relay, and bth relay-to-destination become time selective in nature. The frequency flat time-selective fading links (say the channel from antenna $w$ and $z$ ) are modeled by the AR1 process, as expressed below [40],

$$
\begin{aligned}
h_{w, z}\left(\boldsymbol{\tau}_{1}\right)= & \rho_{w, z} h_{w, z}\left(\boldsymbol{\tau}_{2}\right) \\
& +\sqrt{1-\rho_{w, z}^{2}} e_{w, z}\left(\boldsymbol{\tau}_{1}\right) ;(w, z) \\
& \in\{(s, d),(s, r),(r, d)\} ;
\end{aligned}
$$

where $\tau_{1}$ and $\tau_{2}$ are two adjacent signaling time periods. The random process $e_{w, z}\left(\tau_{1}\right)$ represents the error term arise due to time-selective behavior of the fading links, modeled as $\mathbf{C N}\left(0, \sigma_{e w z}^{2}\right)$. where $\rho_{w, z}$ represents the channel correlation coefficients, modeled by using classical Jake's model $\rho_{w, z}=J_{0}\left\{2 \pi f_{c} v_{w, z} / R_{\mathrm{S}} c\right\}$, where $c, f_{\mathrm{c}}, v_{m}, R_{\mathrm{S}}$ and $J_{0}($.) denote the velocity of light, carrier frequency, node 
velocity, symbol transmission rate, and Bessel function of zeroth order, and first kind, respectively. Similar to works $[40,41]$, the variation in the OSTBC code word matrix within a block for $b$ th relay-to-destination, source-to- $b$ th relay, and SD fading links is modeled as

$A_{\mathrm{RD}}^{(b)}\left(\boldsymbol{\tau}_{1}\right)=\rho_{\mathrm{RD}}^{(b)} A_{\mathrm{RD}}^{(b)}\left(\boldsymbol{\tau}_{2}\right)+\sqrt{1-\left(\rho_{\mathrm{RD}}^{(b)}\right)^{2}} \mathfrak{J}_{\mathrm{RD}}^{(b)}\left(\boldsymbol{\tau}_{1}\right)$,

$A_{\mathrm{SR}}^{(b)}\left(\boldsymbol{\tau}_{1}\right)=\rho_{\mathrm{SR}}^{(b)} A_{\mathrm{SR}}^{(b)}\left(\boldsymbol{\tau}_{2}\right)+\sqrt{1-\rho_{\mathrm{SR}}^{(b)}} \Im_{\mathrm{SR}}^{(b)}\left(\boldsymbol{\tau}_{1}\right)$

$A_{\mathrm{SD}}\left(\boldsymbol{\tau}_{1}\right)=\rho_{\mathrm{SD}} A_{\mathrm{SD}}\left(\boldsymbol{\tau}_{2}\right)+\sqrt{1-\rho_{\mathrm{SD}}^{2}} \Im_{\mathrm{SD}}\left(\boldsymbol{\tau}_{1}\right)$,

where $\mathfrak{J}_{\mathrm{SR}}^{(b)}\left(\boldsymbol{\tau}_{1}\right), \mathfrak{\Im}_{\mathrm{RD}}^{(b)}\left(\boldsymbol{\tau}_{1}\right)$ and $\mathfrak{\Im}_{\mathrm{SD}}\left(\boldsymbol{\tau}_{1}\right)$ denote the error matrices arise due to time-selective nature of the fading links. In non-cooperative wireless transmission over time-selective fading, the MIMO-OSTBC code word matrix is no longer orthogonal, classical Alamouti decoder is a no longer optimal decoder, and due to interference between obtained decision statistics, system performance decreases. Similar to works $[40,41]$, it has been assumed that due to time-selective Nakagami- $m$ fading links, the destination tracking loops are not able to catch up with their timevarying channel variances, and hence, it only can estimate the ones over the first signaling period of each transmitted block (i.e., $A_{\mathrm{SR}}^{(b)}(1), A_{\mathrm{RD}}^{(b)}(1)$ and $\left.A_{\mathrm{SD}}(1)\right)$ as $\hat{A}_{\mathrm{SR}}^{(b)}(1), \hat{A}_{\mathrm{RD}}^{(b)}(1)$ and $\hat{A}_{\mathrm{SD}}(1)$, respectively. Also, assuming imperfect CSI conditions, $\hat{A}_{\mathrm{SR}}^{(b)}(1), \hat{A}_{\mathrm{RD}}^{(b)}(1)$ and $\hat{A}_{\mathrm{SD}}(1)$ can be expressed as $\hat{A}_{\mathrm{SR}}^{(b)}(1)=A_{\mathrm{SR}}^{(b)}(1)+A_{\in \mathrm{SR}}^{(b)}(1), \hat{A}_{\mathrm{RD}}^{(b)}(1)=A_{\mathrm{RD}}^{(b)}(1)+A_{\in \mathrm{RD}}^{(b)}(1)$ and $\hat{A}_{\mathrm{SD}}(1)=A_{\mathrm{SD}}(1)+A_{\in \mathrm{SD}}(1)$, respectively, where $A_{\in S R}^{(b)}(1), A_{\in R D}^{(b)}(1)$ and $A_{\in S D}(1)$ are channel error matrices, comprised of entries, and each entry is distributed as ZMCSCG with a density of $\mathbf{C N}\left(0, \sigma_{\in w z}^{2}\right)$.

\section{Analysis of DH dual-phase S-DF relaying network}

\subsection{PEP analysis}

In this section, we consider the system model described in previous section and derive the instantaneous SNR expressions $\gamma_{\mathrm{SD}}(t), \gamma_{\mathrm{SR}}^{(b)}(t)$ and $\gamma_{\mathrm{RD}}^{(b)}(t)$ for the SD, source-to- $b$ th relay and $b$ th relay-to-destination fading links, respectively. The schematic representation of $\mathrm{DH}$ relaying protocol is given in Fig. 3. To mathematically demonstrate the effect of imperfect CSI and nodes' mobility on the destination SNRs, we first derive, using (6), the following relationship [41],

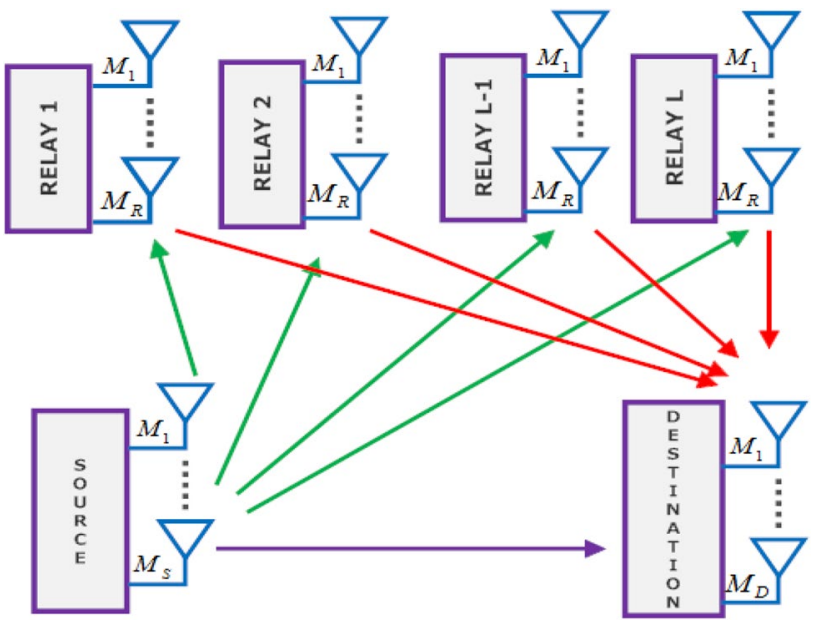

Fig. 3 Schematic diagram of $\mathrm{DH}$, dual-phase, multiple relay $W(0)$ S-DF cooperative communication protocol $[38,39]$

$A_{\mathrm{SD}}(t)=\rho_{\mathrm{SD}}^{t-1} \hat{A}_{\mathrm{SD}}(1)+\rho_{\mathrm{SD}}^{t-1} A_{\mathrm{ESD}}(1)-\underbrace{\sqrt{1-\rho_{\mathrm{SD}}^{2}} \sum_{i=1}^{t-1} \rho_{\mathrm{SD}}^{t-i-1} \mathfrak{\Im}_{\mathrm{SD}}(i)}_{\Psi_{\mathrm{SD}}(t)}$.

where node mobility term $\Psi_{\mathrm{SD}}(t)=0 \Rightarrow \rho_{\mathrm{SD}}=1$. Substituting (7) into (2), $R_{\mathrm{SD}}(t)$ can be written as

$$
\begin{aligned}
R_{\mathrm{SD}}(t)= & \sqrt{P_{\mathrm{S}} \mathbf{c}_{0} / M R_{\mathrm{C}}}\left\{\rho_{\mathrm{SD}}^{t-1} \hat{A}_{\mathrm{SD}}(1) B_{\mathrm{S}}(t)\right. \\
& \left.+\rho_{\mathrm{SD}}^{t-1} A_{\in \mathrm{SD}}(1) B_{\mathrm{S}}(t)-B_{\mathrm{S}}(t) \boldsymbol{\Psi}_{\mathrm{SD}}(t)\right\}+\mathbb{N}_{\mathrm{SD}}(t),
\end{aligned}
$$

and further mathematical arrangement leads to

$$
\begin{aligned}
R_{\mathrm{SD}}(t)= & \underbrace{\rho_{\mathrm{SD}}^{t-1} \hat{A}_{\mathrm{SD}}(1) B_{\mathrm{S}}(t) \sqrt{\mathbf{c}_{0} P_{\mathrm{S}} / M R_{\mathrm{C}}}}_{\text {DESIRED SIGNAL }} \\
& +\underbrace{\sqrt{P_{\mathrm{S}} \mathbf{c}_{0} / M R_{\mathrm{C}}} \rho_{\mathrm{SD}}^{t-1} A_{\in \mathrm{SD}}(1) B_{\mathrm{S}}(t)}_{\text {IMPERFECT CSI NOISE }} \\
& +\underbrace{\mathbb{N}_{\mathrm{SD}}(t)}_{\text {WHITE NOISE }}-\underbrace{\sqrt{P_{\mathrm{S}} \mathbf{c}_{0} / M R_{\mathrm{C}}} B_{\mathrm{S}}(t) \Psi_{\text {SD }}(t)}_{\text {NODES MOBILTY NOISE }} .
\end{aligned}
$$

Since $A_{\in S D}(1), \mathbb{N}_{S D}(t)$ and $\Im_{S D}(i)$ are ZMCSCG random processes (RPs), the addition of noise terms in (8) will also ZMCSCG RPs and with a variance of $\mathbb{N}_{\text {eff }}^{\mathrm{SD}}=\left(N_{0}+\rho_{\mathrm{SD}}^{2(t-1)} \sigma_{\in \mathrm{SD}}^{2}+\left(1-\rho_{\mathrm{SD}}^{2(t-1)}\right) \sigma_{e \mathrm{SD}}^{2}\right)[41,42]$. Since Alamouti STBC converts MIMO channels into orthogonal channels [43], from (8) and by utilizing $\mathbb{N}_{\text {eff' }}^{\text {SD }}$ one can obtain the instantaneous SNR $\gamma_{S D}(t)$ for SD fading link as

$\gamma_{\mathrm{SD}}(t)=\mathbf{c}_{0} \gamma_{\mathrm{SD}}^{a v}\left\|\hat{A}_{\mathrm{SD}}(1)\right\|^{2}$ 
where $\gamma_{\mathrm{SD}}^{a v}$ is expressed below

$\gamma_{\mathrm{SD}}^{a v}=\mid \rho_{\mathrm{SD}}^{2(t-1)}\left\{1+\mid \rho_{\mathrm{SD}}^{2(t-1)} \kappa \sigma_{\in \mathrm{SD}}^{2}+\mathrm{I}\left(1-\rho_{\mathrm{SD}}^{2(t-1)}\right) \kappa \sigma_{e S D}^{2}\right\}^{-1}$.

where $\mathrm{I}=P_{\mathrm{S}} /\left(N_{0} M R_{\mathrm{C}}\right)$ and $\kappa$ denotes the number of nonzero complex modulated symbol transmission per Alamouti OSTBC code word.

Applying the similar approach, one can derive the instantaneous SNR expressions $\gamma_{\mathrm{SR}}^{(b)}(t)$ and $\gamma_{\mathrm{RD}}^{(b)}(t)$ for source-to- $b$ th relay and $b$ th relay-to-destination fading links, respectively, as [43],

$\gamma_{\mathrm{SR}}^{(b)}(t)=\mathbf{c}_{2} \gamma_{\mathrm{SR}}^{a v}\left\|\hat{A}_{\mathrm{SR}}^{(b)}(1)\right\|^{2}, \quad \gamma_{\mathrm{RD}}^{(b)}(t)=\mathbf{c}_{1} \gamma_{\mathrm{RD}}^{a v}\left\|\hat{A}_{\mathrm{RD}}^{(b)}(1)\right\|^{2}$,

where $\gamma_{\mathrm{SR}}^{a v}$ and $\gamma_{\mathrm{RD}}^{a v}$ are expressed in (10) and (11), respectively [43],
Table 1 Modulation-dependent parameters for various modulation schemes

\begin{tabular}{llll}
\hline Modulation schemes & $a^{\prime}$ & $b^{\prime}$ & $c^{\prime}$ \\
\hline M-PSK & 2 & $2 \sin ^{2}\left(\frac{\pi}{M}\right)$ & 0 \\
BPSK & 1 & 2 & 0 \\
BFSK & 1 & 1 & 0 \\
M-PAM & $2 \frac{(M-1)}{M}$ & $\frac{6}{M^{2}-1}$ & 0 \\
MSK or QPSK & 2 & 2 & 1 \\
Coherent DPSK & 2 & 2 & 2 \\
M-QAM & $4 \frac{(\sqrt{M}-1)}{\sqrt{M}}$ & $\frac{3}{M-1}$ & $4\left(\frac{\sqrt{M}-1}{\sqrt{M}}\right)^{2}$
\end{tabular}

$\gamma_{\mathrm{SR}}^{a v}=I\left(\rho_{\mathrm{SR}}^{(b)}\right)^{2(t-1)}\left\{1+I\left(\rho_{\mathrm{SR}}^{(b)}\right)^{2(t-1)} \kappa\left(\sigma_{\in \mathrm{SR}}^{(b)}\right)^{2}+I\left(1-\left(\rho_{\mathrm{SR}}^{(b)}\right)^{2(t-1)}\right) \kappa\left(\sigma_{e \mathrm{SR}}^{(b)}\right)^{2}\right\}^{-1}$

$$
\begin{aligned}
\gamma_{\mathrm{RD}}^{a v}= & I^{\prime}\left(\rho_{\mathrm{RD}}^{(b)}\right)^{2(t-1)}\left\{1+I^{\prime}\left(\rho_{\mathrm{RD}}^{(b)}\right)^{2(t-1)} \kappa\left(\sigma_{\in \mathrm{RD}}^{(b)}\right)^{2}\right. \\
& \left.+l^{\prime}\left(1-\left(\rho_{\mathrm{RD}}^{(b)}\right)^{2(t-1)}\right) \kappa\left(\sigma_{e \mathrm{RD}}^{(b)}\right)^{2}\right\}^{-1}
\end{aligned}
$$

where $l^{\prime}=P_{b} /\left(N_{0} M R_{C}\right)$. Let $P$ denote the total available power budget, one can write $P_{\mathrm{S}}$ and $P_{b}$ in terms of $P$ as $P_{\mathrm{S}}+\sum_{b=1}^{L} P_{b} \leq P$. The average end-to-end error probability expression for the error event when $B_{s}(t)$ was confused with $B_{l}(t) \in \mathbb{C}^{M \times T_{s}}$ can be evaluated by averaging the conditional error probability of source-to-bth relay over the probability density function (PDF) of received instantaneous $\operatorname{SNR} \gamma_{\mathrm{SR}}^{(b)}(t)$. For averaging, the conditional probability for the error event $B_{s}(t) \rightarrow B_{l}(t)$ moment-generating function (MGF) [44]-based approach is used in this work and can be expressed as performing the singular value decomposition (SVD) [45] on the code word difference matrix $B_{s}(t)-B_{l}(t)$, and $\left(\tilde{\sigma}_{s, r}^{b}\right)^{2}=\left(\rho_{\mathrm{SR}}^{(b)}\right)^{2(t-1)}\left(\sigma_{s, r}^{b}\right)^{2}+\left(1-\left(\rho_{\mathrm{SR}}^{(b)}\right)^{2(t-1)}\right)\left(\sigma_{\in \mathrm{SR}}^{(b)}\right)^{2}$. The average end-to-end PEP can be derived by evaluating $\boldsymbol{\Xi}_{1}$ and $\boldsymbol{\Xi}_{2}$. Substituting (13) into (12), $\boldsymbol{\Xi}_{1}$ and $\boldsymbol{\Xi}_{2}$ are expressed below

$$
\begin{aligned}
& \boldsymbol{\Xi}_{1}=\frac{a^{\prime}}{\pi} \int_{0}^{\pi / 2}\left(\frac{\tau_{\mathrm{SR}}}{\tau_{\mathrm{SR}}+\frac{\gamma_{\mathrm{SR}}^{a v} \mathbf{c}_{2} \lambda_{l}^{2} b^{\prime}\left(\tilde{\sigma}_{s, r}^{b}\right)^{2}}{2 \sin ^{2} \theta}}\right)^{\tau_{\mathrm{SR}}} \mathrm{d} \theta, \\
& \boldsymbol{\Xi}_{2}=\frac{c^{\prime}}{\pi} \int_{0}^{\pi / 4}\left(\frac{\tau_{\mathrm{SR}}}{\tau_{\mathrm{SR}}+\frac{\gamma_{\mathrm{SR}}^{a v} \lambda_{l}^{2} \mathbf{c}_{2} b^{\prime}\left(\tilde{\sigma}_{\mathrm{S}, r}^{b}\right)^{2}}{2 \sin ^{2} \theta}}\right)^{\tau_{\mathrm{SR}}} \mathrm{d} \theta .
\end{aligned}
$$

where $\theta$ is integration variable and $\mathrm{M}_{\gamma_{\mathrm{SR}}^{(b)}(t)}(\cdot)$ denotes the MGF, defined as

$\mathrm{M}_{\gamma_{\mathrm{SR}}^{(b)}(t)}(s)=\left(\frac{\tau_{\mathrm{SR}}}{\tau_{\mathrm{SR}}+\left(\tilde{\sigma}_{s, r}^{b}\right)^{2} \gamma_{\mathrm{SR}}^{\mathrm{av}} \mathbf{c}_{2} \lambda^{2} s}\right)^{\tau_{\mathrm{SR}}}$,

where $\tau_{\mathrm{SR}}=m_{\mathrm{SR}} M^{2}$ and $a^{\prime}, b^{\prime}$ and $c^{\prime}$ are modulationdependent parameters listed in Table $1, \lambda_{l}, 1 \leq I \leq|\Upsilon|$ denote the nonzero singular values (SVs) obtain by
1. Evaluation of $\Xi_{1}$

For solving $\Xi_{1}$, let us change the variable by substitution $\cos ^{2} \theta=\Theta$, and this leads to $\sin ^{2} \theta=1-\Theta$ and $-2 \sin \theta \cos \theta \mathrm{d} \theta=\mathrm{d} \Theta$. Thus, the limits of the integral would change from 0 to 1 and the integrating variable $\mathrm{d} \theta$ changes to 
$\mathrm{d} \theta=\frac{-\mathrm{d} \Theta}{2 \sqrt{\Theta} \sqrt{1-\Theta}}$.

Therefore, $\Xi_{1}$ can now be given as

$\Xi_{1}=\frac{a^{\prime}}{\pi} \int_{0}^{1}\left(\frac{\tau_{\mathrm{SR}}}{\tau_{\mathrm{SR}}+\frac{\mathbf{c}_{2} b^{\prime} \lambda_{1}^{2} \gamma_{\mathrm{SR}}^{a v}\left(\tilde{\sigma}_{S, r}^{b}\right)^{2}}{(1-\Theta)}}\right)^{\tau_{\mathrm{SR}}} \frac{\mathrm{d} \Theta}{2 \sqrt{\Theta} \sqrt{1-\Theta}}$.
2. Evaluation of $\Xi_{2}$

Following the similar approach adopted for evaluation of $\Xi_{1}$, to solve $\Xi_{2}$, let us change the variable by substituting $\sin ^{2} \theta=\Theta / 2$, and this will lead to

$2 \sin \theta \cos \theta d \theta=\frac{d \Theta}{2}$

Further, $\Xi_{1}$ can be written as

$$
\begin{aligned}
\Xi_{1} & =\frac{a^{\prime}}{\pi} \int_{0}^{1}\left(\tau_{\mathrm{SR}}(1-\Theta)\right)^{\tau_{\mathrm{SR}}}\left(\tau_{\mathrm{SR}}(1-\Theta)+\mathbf{c}_{2} b^{\prime} \lambda_{l}^{2} \gamma_{\mathrm{SR}}^{a v}\left(\tilde{\sigma}_{s, r}^{b}\right)^{2}\right)^{-\tau_{\mathrm{SR}}} \frac{\mathrm{d} \Theta}{2 \sqrt{\Theta} \sqrt{1-\Theta}}, \\
& =\frac{a^{\prime}}{\pi} \int_{0}^{1} \frac{(1-\Theta)^{\tau_{\mathrm{SR}}-1 / 2}\left(\tau_{\mathrm{SR}}\right)^{\tau_{\mathrm{SR}}}}{2 \sqrt{\Theta}}\left(\tau_{\mathrm{SR}}+\mathbf{c}_{2} b^{\prime} \lambda_{l}^{2} \gamma_{\mathrm{SR}}^{a v}\left(\tilde{\sigma}_{s, r}^{b}\right)^{2}-\tau_{\mathrm{SR}} \Theta\right)^{-\tau_{\mathrm{SR}}} \mathrm{d} \Theta .
\end{aligned}
$$

Furthermore, (15) can be written as

$$
\begin{aligned}
& \Xi_{1}=\frac{a^{\prime}}{\pi} \int_{0}^{1} \frac{(1-\Theta)^{\tau_{\mathrm{SR}}-1 / 2}\left(\tau_{\mathrm{SR}}\right)^{\tau_{\mathrm{SR}}}}{2 \sqrt{\Theta}}\left(\tau_{\mathrm{SR}}+\mathbf{c}_{2} b^{\prime} \lambda_{l}^{2} \gamma_{\mathrm{SR}}^{a v}\left(\tilde{\sigma}_{S, r}^{b}\right)^{2}\right)^{-\tau_{\mathrm{SR}}}\left(1-\frac{\Theta}{1+\frac{\mathbf{c}_{2} b^{\prime} \lambda_{l}^{2} \gamma_{\mathrm{SR}}^{a v}\left(\tilde{\sigma}_{S, r}^{b}\right)^{2}}{\tau_{\mathrm{SR}}}}\right)^{-\tau_{\mathrm{SR}}} \mathrm{d} \Theta, \\
& =\frac{a^{\prime}}{\pi}\left(\frac{2 \tau_{\mathrm{SR}}}{2 \tau_{\mathrm{SR}}+2 \mathbf{c}_{2} b^{\prime} \lambda_{l}^{2} \gamma_{\mathrm{SR}}^{a v}\left(\tilde{\sigma}_{S, r}^{b}\right)^{2}}\right)^{\tau_{\mathrm{SR}}} \int_{0}^{1} \frac{(1-\Theta)^{\tau_{\mathrm{SR}}-1 / 2} \Theta^{-1 / 2}}{2}\left(1-\frac{\Theta}{1+\frac{\left.2 \mathbf{c}_{2} b^{\prime} \lambda_{l}^{2} \gamma_{\mathrm{SR}}^{a(} \tilde{\sigma}_{S, r}^{b}\right)^{2}}{2 \tau_{\mathrm{SR}}}}\right)^{-\tau_{\mathrm{SR}}} \mathrm{d} \Theta .
\end{aligned}
$$

The expression given above can be represented in the form of Gauss hypergeometric function [46], defined as

${ }_{2} F_{1}\left(a_{1}, b_{1} ; c_{1} ; x\right)=\frac{\Gamma\left(c_{1}\right)}{\Gamma\left(c_{1}-a_{1}\right) \times \Gamma\left(a_{1}\right)} \int_{0}^{1} \Theta^{b_{1}-1}(1-\Theta)^{c_{1}-b_{1}-1}(1-\Theta x)^{-a_{1}} \mathrm{~d} \Theta$.

Comparing (19) with (18), the parameters can be obtained

as

$a_{1}=\tau_{\mathrm{SR}}, b_{1}=0.50, c_{1}=1+\tau_{\mathrm{SR}}$ and $x=1-\left(1+\frac{\lambda_{1}^{2} 2 b^{\prime} \mathbf{c}_{2} \gamma_{\mathrm{SR}}^{a v}\left(\tilde{\sigma}_{s, \mathrm{r}}^{b}\right)^{2}}{2 \tau_{\mathrm{SR}}}\right)^{-1}$.

Using the results obtained above, $\Xi_{1}$ can be written as

$$
\Xi_{1}=\frac{a^{\prime}}{2 \pi}\left(\frac{2 \tau_{\mathrm{SR}}}{2 \tau_{\mathrm{SR}}+\left(\tilde{\sigma}_{S, r}^{b}\right)^{2} b^{\prime} \lambda_{l}^{2} \mathbf{c}_{2} \gamma_{\mathrm{SR}}^{a v}}\right)^{\tau_{\mathrm{SR}}} \frac{\Gamma\left(\frac{1}{2}\right) \Gamma\left(\tau_{\mathrm{SR}}\right)}{\Gamma\left(\tau_{\mathrm{SR}}+1\right)} \times{ }_{2} F_{1}\left(\tau_{\mathrm{SR}} \frac{1}{2} ; \tau_{\mathrm{SR}}+1 ; 1-\frac{1}{1+\frac{\left(\tilde{\sigma}_{\mathrm{S}, \mathrm{r}}^{b}\right)^{2} b^{\prime} \lambda_{l}^{2} \mathbf{c}_{2} \gamma_{\mathrm{SR}}^{a v}}{2 \tau_{\mathrm{SR}}}}\right) .
$$




$$
\mathrm{d} \theta=\frac{\mathrm{d} \Theta}{4 \sqrt{\frac{\Theta}{2}} \sqrt{1-\frac{\Theta}{2}}}
$$

The limits of integral would change from 0 to $1 ; \Xi_{2}$ can be expressed as

$$
\begin{aligned}
\Xi_{2} & =\frac{c^{\prime}}{\pi} \int_{0}^{1}\left(\frac{\tau_{\mathrm{SR}}}{\tau_{\mathrm{SR}}+\frac{\left(\tilde{\sigma}_{S, r}^{b}\right)^{2} b^{\prime} \lambda_{l}^{2} \gamma_{\mathrm{SR}}^{a v} \mathbf{c}_{2}}{\Theta}}\right)^{\tau_{\mathrm{SR}}} \frac{\mathrm{d} \Theta}{4 \sqrt{\frac{\Theta}{2}} \sqrt{1-\frac{\Theta}{2}}} \\
& =\frac{c^{\prime}}{2 \sqrt{2 \pi}} \int_{0}^{1}\left(1+\frac{\left(\tilde{\sigma}_{s, r}^{b}\right)^{2} b^{\prime} \lambda_{l}^{2} \gamma_{\mathrm{SR}}^{a v} \mathbf{c}_{2}}{\tau_{\mathrm{SR}}}\right)^{-\tau_{\mathrm{SR}}} \frac{\mathrm{d} \Theta}{4 \sqrt{\frac{\Theta}{2}} \sqrt{1-\frac{\Theta}{2}}} \\
& =\frac{c^{\prime}}{2 \sqrt{2 \pi}} \int_{0}^{1} \Theta^{\tau_{\mathrm{SR}}-\frac{1}{2}}\left(\Theta+\frac{\left(\tilde{\sigma}_{S, r}^{b}\right)^{2} b^{\prime} \lambda_{l}^{2} \gamma_{\mathrm{SR}}^{a v} \mathbf{c}_{2}}{\tau_{\mathrm{SR}}}\right)^{-\tau_{\mathrm{SR}}} \frac{\mathrm{d} \Theta}{\sqrt{1-\frac{\Theta}{2}}} .
\end{aligned}
$$

The expression given in (20) is compared with Appell hypergeometric function [46] of two variables, defined as
OSTBC code word $B_{s}(t)$ and $\xi_{j}^{t}(b)=0$ if the $b$ th relay incorrectly decodes $B_{s}(t)$. For simplicity, we represented $\xi_{j}^{t}(b)$ by an integer decimal number as [39], $\xi_{j}^{t}=\left[\xi_{j}^{t}(1), \xi_{j}^{t}(2), \ldots, \xi_{j}^{t}(L)\right]^{T}, 0 \leq j \leq 2^{L}-1$. The state vectors when all the relays correctly and incorrectly decode the $t$ th OSTBC code word are given as $\xi_{2^{L}-1}^{t}=[1,1,1, \ldots, 1]^{T}$ and $\xi_{q}^{t}=[0,0,0, \ldots, 0]^{T}$, respectively. Further, let the set $\psi_{j}^{t}=\left\{b \mid \xi_{j}^{t}(b)=1, b=1,2, \ldots, L\right\}$ consist all the correctly decoding relay nodes. The conditional PEP for the erroneous event $B_{s}(t) \rightarrow B_{i}(t)$ when code word $B_{s}(t)$ was confused with code word $B_{i}(t)$ at the destination node conditioned on the instantaneous SNR $\gamma ; \gamma=\left\{\gamma_{\mathrm{SD}}(t), \gamma_{\mathrm{SR}}^{(b)}(t), \gamma_{\mathrm{RD}}^{(b)}(t)\right\}$ is expressed as [48],

$$
P_{b}\left(B_{s}(t) \rightarrow B_{i}(t) / \gamma\right)=\sum_{j=0}^{2^{L}-1} P_{b}\left(B_{s}(t) \rightarrow B_{i}(t) \mid \xi_{j}^{t}, \gamma\right) P_{b}\left(\xi_{j}^{t} / \gamma\right)
$$

where $P_{b}\left(\xi_{j}^{t} / \gamma\right)$ represents the instantaneous probability

$F_{A}^{(1)}\left(\chi_{1} ; l_{1}, l_{1}^{\prime} ; \gamma^{\prime \prime} ; x^{\prime}, y^{\prime}\right)=\frac{\Gamma\left(\gamma^{\prime \prime}\right)}{\Gamma\left(\chi_{1}\right) \Gamma\left(\gamma^{\prime \prime}-\chi_{1}\right)} \int_{0}^{1} \Theta^{\chi_{1}-1}(1-\Theta)^{\gamma^{\prime \prime}-\chi_{1}-1}\left(1-\Theta x^{\prime}\right)^{-l_{1}}\left(1-\Theta y^{\prime}\right)^{-l_{1}^{\prime}} \mathrm{d} \Theta$

Comparing the expression given in (20) with the definition of the Appell hypergeometric function, various parameters can be obtained as that the wireless network conditioned on the Gamma distributed SNR $\gamma$ is in state $\xi_{j}^{t}$ and $P_{b}\left(B_{s}(t) \rightarrow B_{i}(t) \mid \xi_{j}^{t}, \gamma\right)$ denotes the instantaneous PEP of wireless network being

$\chi_{1}=\tau_{\mathrm{SR}}+\frac{1}{2}, l_{1}=\frac{1}{2}, l_{1}^{\prime}=\tau_{\mathrm{SR}}, \gamma^{\prime \prime}=\tau_{\mathrm{SR}}+\frac{3}{2}, x^{\prime}=\frac{1}{2}$ and $y^{\prime}=\frac{-\tau_{\mathrm{SR}}}{\left(\tilde{\sigma}_{s, r}^{b}\right)^{2} b^{\prime} \lambda_{l}^{2} \gamma_{\mathrm{SR}}^{a v} \mathbf{c}_{2}}$.

Thus, $\Xi_{2}$ can be expressed in the form of Appell hypergeometric function of two variables as in state $\xi_{j}^{t}$ conditioned on the SNR $\gamma$. Applying expectation operator $E_{\gamma}\{$.$\} over the SNR \gamma$ along with the assumption

$\Xi_{2}=\frac{c^{\prime}}{2 \sqrt{2 \pi}}\left(\frac{\left(\tilde{\sigma}_{s, r}^{b}\right)^{2} b^{\prime} \lambda_{l}^{2} \gamma_{\mathrm{SR}}^{a v} \mathbf{c}_{2}}{\tau_{\mathrm{SR}}}\right)^{\tau_{\mathrm{SR}}} \frac{\Gamma\left(\tau_{\mathrm{SR}}+\frac{1}{2}\right)}{\Gamma\left(\tau_{\mathrm{SR}}+\frac{3}{2}\right)} \times \mathbf{F}_{\mathrm{A}}^{(1)}\left(\tau_{\mathrm{SR}}+\frac{1}{2} ; \frac{1}{2}, \tau_{\mathrm{SR}} ; \tau_{\mathrm{SR}}+\frac{3}{2} ; \frac{1}{2} ;-\frac{\tau_{\mathrm{SR}}}{\left(\tilde{\sigma}_{s, r}^{b}\right)^{2} b^{\prime} \lambda_{l}^{2} \gamma_{\mathrm{SR}}^{a v} \mathbf{c}_{2}}\right)$

Now using $\Xi_{1}$ and $\Xi_{2}$, average PEP expression for sourceto- $b$ th relay fading link $\bar{P}_{\mathrm{E}}^{S R}\left(B_{s}(t) \rightarrow B_{l}(t)\right)$ can be evaluated analytically via the CF expressions of (19) and (22).

Nonetheless, these solutions are in the form of the Appell hypergeometric function and Gauss hypergeometric function. These functions can be computed using software tools like Mathematica and MATLAB [47].For a given OSTBC code word transmission, there are two possible states for the $b$ th relay; either it will decode successfully or unsuccessfully. Let us define a vector $\xi_{j}^{t}(b)$ denotes the states of the first $b$ relays for a given OSTBC code word transmission, $\xi_{j}^{t}(b)=1$ if $b$ th relay correctly decodes the

SN Applied Sciences that fading links are i.n.i.d., the average PEP of the erroneous event $B_{s}(t) \rightarrow B_{i}(t)$ at the receiving terminal can be expressed as $[48,49]$,

$P_{b}\left(B_{s}(t) \rightarrow B_{i}(t)\right)=\sum_{j=0}^{2^{L}-1} E_{\gamma}\left\{P_{b}\left(B_{s}(t) \rightarrow B_{i}(t) / \xi_{j}^{t}, \gamma\right)\right\} E_{\gamma}\left\{P_{b}\left(\xi_{j}^{t} / \gamma\right)\right\}$ 
Since we consider that the source-to-bth relay fading links are i.n.i.d., expression given in (27) can be further simplified as
Substituting (29) into (28), the average error probability expression for the relaying network being in the state $\xi_{j}^{t}(b)$ is given as

$P_{b}\left(B_{s}(t) \rightarrow B_{i}(t)\right)=\sum_{j=0}^{2^{L}-1} E_{\gamma}\left\{P_{b}\left(B_{s}(t) \rightarrow B_{i}(t) / \xi_{j}^{t}, \gamma\right)\right\} \times \prod_{b=1}^{L} E_{\gamma}\left\{P_{b}\left(\xi_{j}^{t}(b) / \gamma\right)\right\}$.

where $E_{\gamma}\left\{P_{b}\left(\xi_{j}^{t}(b) / \gamma\right)\right\}$ denotes the expected probability of $b$ th relay being in the state $\xi_{j}^{t}(b)$ and can be shown in relations of expected PEP of erroneous event $B_{s}(t) \rightarrow B_{l}(t)$ is expressed as [48, 49],

$E_{\gamma}\left\{P_{b}\left(\xi_{j}^{t}(b) / \gamma\right)\right\}= \begin{cases}\sum_{l=1}^{|\Upsilon|}\left(\Xi_{1}-\Xi_{2}\right) ; & \text { if } \xi_{j}^{t}(b)=0 \\ 1-\sum_{l=1}^{|Y|}\left(\Xi_{1}-\Xi_{2}\right) ; & \text { if } \xi_{j}^{t}(b)=1\end{cases}$

$$
\begin{aligned}
& E_{\gamma}\left\{P_{b}\left(\xi_{j}^{t}(b) / \gamma\right)\right\}=\prod_{b \in \bar{\psi}_{j}^{t}}\left\{\sum_{l=1}^{|\mathrm{Y}|}\left(\Xi_{1}-\Xi_{2}\right)\right\} \\
& \quad \times \prod_{b \in \psi_{j}^{t}}\left\{1-\sum_{l=1}^{|\mathrm{Y}|}\left(\Xi_{1}-\Xi_{2}\right)\right\} .
\end{aligned}
$$

The set $b \in \psi_{j}^{t}$ and $b \in \bar{\psi}_{j}^{t}$ includes all the relays, which decodes the code word matrix $B_{s}(t)$ successfully and unsuccessfully, respectively. Let us consider each relay is transmitted from set $\psi_{j}^{t}$, at the destination node, instantaneous PEP of the erroneous event $B_{s}(t) \rightarrow B_{i}(t)$, when the system is in state $\xi_{j}^{t}$ is given as [48],

$$
\begin{aligned}
P_{b}\left(B_{s}(t) \rightarrow B_{i}(t) / \xi_{j}^{t}, \gamma\right)= & a^{\prime} \mathbb{Q}\left(\sqrt{\left.b^{\prime}\left\{\frac{P_{\mathrm{S}} \rho_{\mathrm{SD}}^{2(t-1)}\left\|\hat{A}_{\mathrm{SD}}(1)\left(B_{\mathrm{S}}(t)-B_{i}(t)\right)\right\|^{2}}{2 M R_{\mathrm{C}} \mathbb{N}_{\mathrm{eff}}^{\mathrm{SD}}}+\sum_{b \in \psi_{j}^{t}} \frac{P_{b}\left(\rho_{\mathrm{RD}}^{(b)}\right)^{2(t-1)}\left\|\hat{A}_{\mathrm{RD}}^{(b)}\left(B_{\mathrm{S}}(t)-B_{i}(t)\right)\right\|^{2}}{2 M R_{\mathrm{C}} \tilde{\mathbb{N}}_{\mathrm{eff}}^{\mathrm{RD}}}\right\}\right)}\right. \\
& -c^{\prime} \mathbb{Q}^{2}\left(\sqrt{b^{\prime}\left\{\frac{P_{\mathrm{S}} \rho_{\mathrm{SD}}^{2(t-1)}\left\|\hat{A}_{\mathrm{SD}}(1)\left(B_{\mathrm{S}}(t)-B_{i}(t)\right)\right\|^{2}}{2 M R_{\mathrm{C}} \mathbb{N}_{\mathrm{eff}}^{\mathrm{SD}}}+\sum_{b \in \psi_{j}^{t}} \frac{P_{b}\left(\rho_{\mathrm{RD}}^{(b)}\right)^{2(t-1)}\left\|\hat{A}_{\mathrm{RD}}^{(b)}\left(B_{\mathrm{S}}(t)-B_{i}(t)\right)\right\|^{2}}{2 M R_{\mathrm{C}} \tilde{\mathbb{N}}_{\mathrm{eff}}^{\mathrm{RD}}}\right\}},\right.
\end{aligned}
$$

$$
\begin{aligned}
P_{b}\left(B_{s}(t) \rightarrow B_{i}(t) / \xi_{j}^{t}, \gamma\right)= & a^{\prime} \mathbb{Q}\left(\sqrt{\left.b^{\prime}\left\{\frac{P_{\mathrm{S}} \rho_{\mathrm{SD}}^{2(t-1)} \lambda_{i}^{2} \sum_{s=1}^{M_{\mathrm{D}}}\left|h_{s, d}(t)\right|^{2}}{2 M R_{\mathrm{C}} \mathbb{N}_{\mathrm{eff}}^{\mathrm{SD}}}+\sum_{b \in \psi_{j}^{t}} \frac{P_{b}\left(\rho_{\mathrm{RD}}^{(b)}\right)^{2(t-1)} \lambda_{i}^{2} \sum_{r=1}^{M_{\mathrm{D}}}\left|h_{r, d}(t)\right|^{2}}{2 M R_{\mathrm{C}} \tilde{\mathbb{N}}_{\mathrm{eff}}^{\mathrm{RD}}}\right\}\right)}\right. \\
& -c^{\prime} \mathbb{Q}^{2}\left(\sqrt{b^{\prime}\left\{\frac{P_{\mathrm{S}} \rho_{\mathrm{SD}}^{2(t-1)} \lambda_{i}^{2} \sum_{s=1}^{M_{\mathrm{D}}}\left|h_{s, d}(t)\right|^{2}}{2 M R_{\mathrm{C}} \mathbb{N}_{\text {eff }}^{\mathrm{SD}}}+\sum_{b \in \psi_{j}^{t}} \frac{P_{b}\left(\rho_{\mathrm{RD}}^{(b)}\right)^{2(t-1)} \lambda_{i}^{2} \sum_{r=1}^{M_{\mathrm{D}}}\left|h_{r, d}(t)\right|^{2}}{2 M R_{C} \tilde{\mathbb{N}}_{\mathrm{eff}}^{\mathrm{RD}}}\right\}},\right.
\end{aligned}
$$

where $\mathbb{Q}($.$) represents the Gaussian Q-function which is the$ tail distribution function of the standard normal distribution [49]. Similar to works [38, 39], after performing SVD of the OSTBC code word difference matrix $B_{S}(t)-B_{i}(t)$, $P_{b}\left(B_{s}(t) \rightarrow B_{i}(t) / \xi_{j}^{t}, \gamma\right)$ given above can be expressed as 
where $\tilde{\mathbb{N}}_{\text {eff }}^{\mathrm{RD}}=\left(N_{0}+\left(\rho_{\mathrm{RD}}^{(b)}\right)^{2(t-1)} \tilde{\sigma}_{\epsilon_{\mathrm{RD}}}^{2}+\left(1-\left(\rho_{\mathrm{RD}}^{(b)}\right)^{2(t-1)}\right) \tilde{\sigma}_{e_{\mathrm{RD}}^{2}}^{2}\right) \cdot \mathrm{SD}$ and $b$ th relay-to-destination instantaneous $\operatorname{SNR} \gamma_{\mathrm{SD}}(t)$ and $\gamma_{\mathrm{RD}}^{(b)}(t)$ cab be expressed as

$\begin{aligned} \gamma_{\mathrm{SD}}(t) & =\frac{P_{\mathrm{S}} \rho_{\mathrm{SD}}^{2(t-1)} \sum_{s=1}^{M_{\mathrm{D}}}\left|h_{s, d}(t)\right|^{2}}{M R_{\mathrm{C}} \mathbb{N}_{\text {eff }}^{\mathrm{SD}}} \text { and } \\ \gamma_{\mathrm{RD}}^{(b)}(t) & =\frac{P_{b}\left(\rho_{\mathrm{RD}}^{(b)}\right)^{2(t-1)} \sum_{r=1}^{N_{\mathrm{D}}}\left|h_{r, d}(t)\right|^{2}}{M R_{\mathrm{C}} \tilde{\mathbb{N}}_{\text {eff }}^{\mathrm{RD}}},\end{aligned}$

respectively.
Using $\gamma_{\mathrm{SD}}(t)$ and $\gamma_{\mathrm{RD}}^{(b)}(t)$ given above, we can express $P_{b}\left(B_{s}(t) \rightarrow B_{i}(t) / \xi_{j}^{t}, \gamma\right)$ as

$$
\begin{aligned}
& P_{b}\left(B_{s}(t) \rightarrow B_{i}(t) / \xi_{j}^{t}, \gamma\right) \\
& =a^{\prime} \mathbb{Q}\left(\sqrt{\left.b^{\prime}\left\{\frac{\lambda_{i}^{2} \gamma_{\mathrm{SD}}(t)}{2}+\sum_{b \in \psi_{j}^{t}} \frac{\lambda_{i}^{2} \gamma_{\mathrm{RD}}^{(b)}(t)}{2}\right\}\right)}\right. \\
& -c^{\prime} \mathbb{Q}^{2}\left(\sqrt{\left.b^{\prime}\left\{\frac{\lambda_{i}^{2} \gamma_{\mathrm{SD}}(t)}{2}+\sum_{b \in \psi_{j}^{t}} \frac{\lambda_{i}^{2} \gamma_{\mathrm{RD}}^{(b)}(t)}{2}\right\}\right)}\right)
\end{aligned}
$$

After averaging the conditional PEP for the error event $B_{s}(t) \rightarrow B_{i}(t)$, given in (31), over the Gamma distributed PDF of the instantaneous SNR's $\gamma_{\mathrm{SD}}(t)$ and $\gamma_{\mathrm{RD}}^{(b)}(t)$, average PEP of error event $B_{s}(t) \rightarrow B_{i}(t)$, can be expressed as

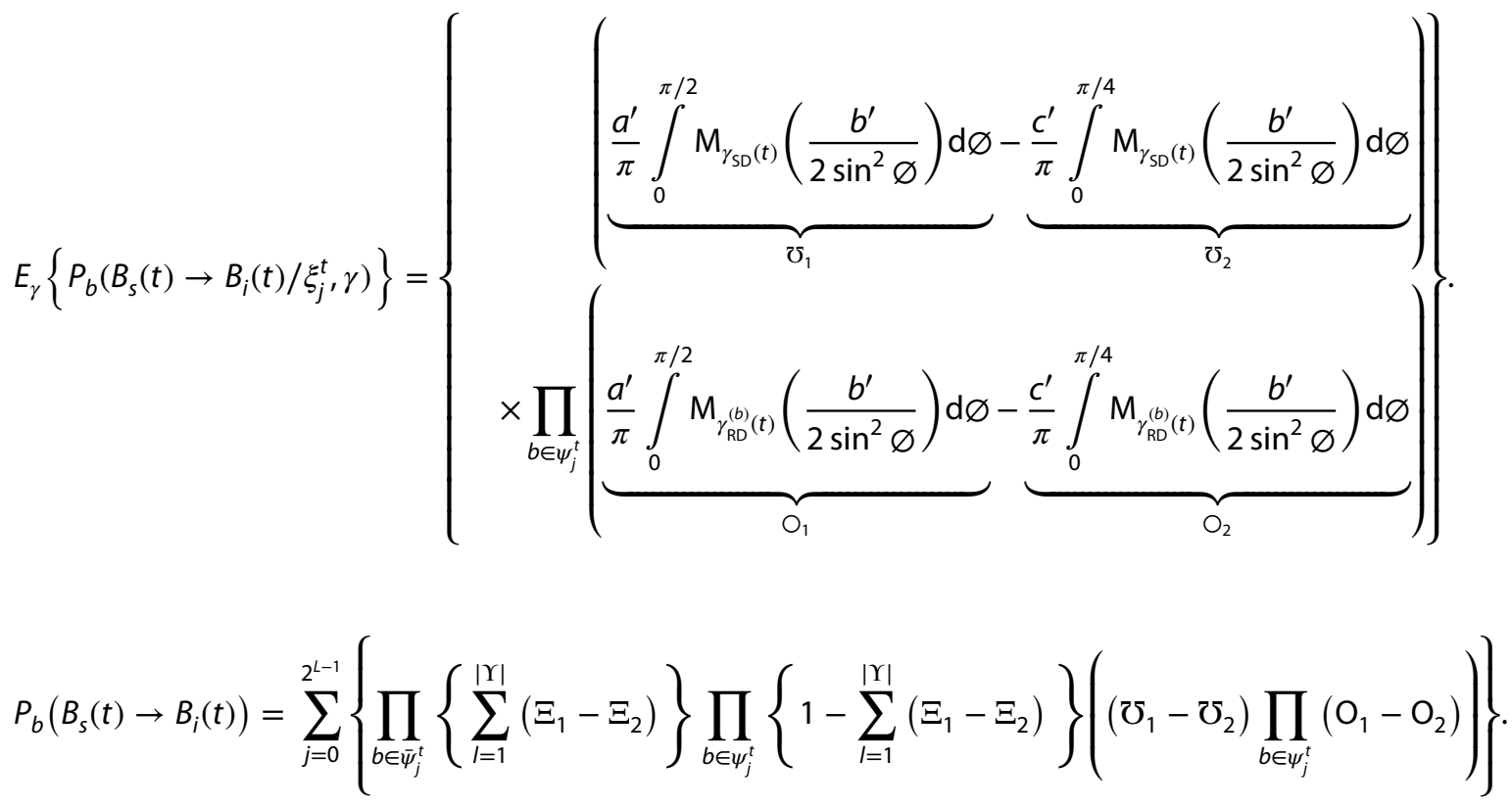

where $\mho_{1}, \mho_{2}, \mathrm{O}_{1}$ and $\mathrm{O}_{2}$ are given below

$$
\begin{aligned}
& \mho_{1}=\frac{a^{\prime}}{2 \pi}\left(\frac{2 \tau_{\mathrm{SD}}}{2 \tau_{\mathrm{SD}}+\tilde{\sigma}_{s, d}^{2} b^{\prime} \lambda_{l}^{2} \mathbf{c}_{0} \gamma_{\mathrm{SD}}^{a v}}\right)^{\tau_{\mathrm{SD}}} \times \frac{\Gamma\left(\frac{1}{2}\right) \Gamma\left(\tau_{\mathrm{SD}}\right)}{\Gamma\left(\tau_{\mathrm{SD}}+1\right)} \times{ }_{2} F_{1}\left(\tau_{\mathrm{SD}}, \frac{1}{2} ; \tau_{\mathrm{SD}}+1 ; 1-\frac{1}{1+\frac{\tilde{\sigma}_{s, d}^{2} b^{\prime} \lambda_{l}^{2} \mathbf{c}_{0} \gamma_{\mathrm{SD}}^{a v}}{2 \tau_{\mathrm{SD}}}}\right), \\
& \mho_{2}=\frac{c^{\prime}}{2 \sqrt{2 \pi}}\left(\frac{\tilde{\sigma}_{s, d}^{2} b^{\prime} \lambda_{l}^{2} \gamma_{\mathrm{SD}}^{a v} \mathbf{c}_{0}}{\tau_{\mathrm{SD}}}\right)^{\tau_{\mathrm{SD}}} \times \frac{\Gamma\left(\tau_{\mathrm{SD}}+\frac{1}{2}\right)}{\Gamma\left(\tau_{\mathrm{SD}}+\frac{3}{2}\right)} \mathbf{F}_{\mathrm{A}}^{(1)}\left(\tau_{\mathrm{SD}}+\frac{1}{2} ; \frac{1}{2}, \tau_{\mathrm{SD}} ; \tau_{\mathrm{SD}}+\frac{3}{2} ; \frac{1}{2} ;-\frac{\tau_{\mathrm{SD}}}{\tilde{\sigma}_{S, d}^{2} b^{\prime} \lambda_{l}^{2} \gamma_{\mathrm{SD}}^{a v} \mathbf{c}_{0}}\right),
\end{aligned}
$$


$\mathrm{O}_{1}=\frac{a^{\prime}}{2 \pi}\left(\frac{2 \tau_{\mathrm{RD}}}{2 \tau_{\mathrm{RD}}+\left(\tilde{\sigma}_{r, d}^{b}\right)^{2} b^{\prime} \lambda_{l}^{2} \mathbf{c}_{1} \gamma_{\mathrm{RD}}^{a v}}\right)^{\tau_{\mathrm{RD}}} \times \frac{\Gamma\left(\frac{1}{2}\right) \Gamma\left(\tau_{\mathrm{RD}}\right)}{\Gamma\left(\tau_{\mathrm{RD}}+1\right)} \times{ }_{2} F_{1}\left(\tau_{\mathrm{RD}}, \frac{1}{2} ; \tau_{\mathrm{RD}}+1 ; 1-\frac{1}{1+\frac{\left(\tilde{\sigma}_{r, d}^{b} b^{\prime} \lambda_{l}^{2} \mathbf{c}_{1} \gamma_{\mathrm{RD}}^{a v}\right.}{2 \tau_{\mathrm{RD}}}}\right)$
$\mathrm{O}_{2}=\frac{c^{\prime}}{2 \sqrt{2 \pi}}\left(\frac{\left(\tilde{\sigma}_{r, d}^{b}\right)^{2} b^{\prime} \lambda_{l}^{2} \gamma_{\mathrm{RD}}^{a v} \mathbf{c}_{1}}{\tau_{\mathrm{RD}}}\right)^{\tau_{\mathrm{RD}}} \frac{\Gamma\left(\tau_{\mathrm{RD}}+\frac{1}{2}\right)}{\Gamma\left(\tau_{\mathrm{RD}}+\frac{3}{2}\right)} \times \mathbf{F}_{\mathrm{A}}^{(1)}\left(\tau_{\mathrm{RD}}+\frac{1}{2} ; \frac{1}{2}, \tau_{\mathrm{RD}} ; \tau_{\mathrm{RD}}+\frac{3}{2} ; \frac{1}{2} ;-\frac{\tau_{\mathrm{RD}}}{\left(\tilde{\sigma}_{r, d}^{b}\right)^{2} b^{\prime} \lambda_{l}^{2} \gamma_{\mathrm{RD}}^{a v} \mathbf{c}_{1}}\right)$.

where $\quad \tilde{\sigma}_{s, d}^{2}=\rho_{\mathrm{SD}}^{2(t-1)} \sigma_{s, d}^{2}+\left(1-\rho_{\mathrm{SD}}^{2(t-1)}\right) \sigma_{\in S \mathrm{~S}^{\prime}}^{2}\left(\tilde{\sigma}_{r, d}^{b}\right)^{2}=\left(\rho_{\mathrm{RD}}^{(b)}\right)^{2(t-1)}$

$\left(\sigma_{r, d}^{b}\right)^{2}+\left(1-\left(\rho_{\mathrm{RD}}^{(b)}\right)^{2(t-1)}\right)\left(\sigma_{\in \mathrm{RD}}^{(b)}\right)^{2}, \tau_{\mathrm{SD}}=m_{\mathrm{SD}} M M_{\mathrm{D}} \quad$ a n d

$\tau_{\mathrm{RD}}=m_{\mathrm{RD}} M M_{\mathrm{D}}$. Lastly, substituting (32) and (30) into (28), average PEP for the error event $B_{s}(t) \rightarrow B_{i}(t)$ is expressed as given in (33).

Further, the per-block average PEP $\bar{P}_{\mathrm{E}}$ is expressed as

$\bar{P}_{\mathrm{E}} \leq \frac{1}{M_{B}} \sum_{t=1}^{M_{B}} \sum_{s=1}^{|\Upsilon|} P_{b}\left(B_{s}(t)\right) \sum_{i=1, s \neq i}^{|\Upsilon|} P_{b}\left(B_{s}(t) \rightarrow B_{i}(t)\right)$,

where the probability of transmission of code word that transmits through the source is given as $P_{b}\left(B_{s}(t)\right)$ and $P_{b}\left(B_{s}(t)\right)=\frac{1}{|\Upsilon|}$ in case of equally likely transmission of all the possible code words from code word set $\Upsilon$.

\subsection{OP Analysis}

Let $P_{b}\left(\bar{\Phi}^{\prime}\right)$ denote the probability of the event $\bar{\Phi}^{\prime}$ when $\gamma_{\mathrm{SR}}^{(b)}(t)<\gamma_{0}$ and $P_{b}\left(\Phi^{\prime}\right)=1-P_{b}\left(\bar{\Phi}^{\prime}\right)$ denote the probability of the event $\Phi^{\prime}$ when $\gamma_{\mathrm{SR}}^{(b)}(t)>\gamma_{0}$. Note that $\gamma_{\mathrm{SD}}(t), \gamma_{\mathrm{SR}}^{(b)}(t)$ and $\gamma_{\mathrm{RD}}^{(b)}(t)$ follow the Erlang distribution [50]. The PDF and CDF for the instantaneous SNR terms $\gamma_{\mathrm{SD}}(t), \gamma_{\mathrm{SR}}^{(b)}(t)$ and $\gamma_{\mathrm{RD}}^{(b)}(t)$ are expressed below

$$
\begin{aligned}
& f_{\gamma_{\mathrm{SD}}(t)}(x)=\frac{\left(\frac{1}{\mathbf{c}_{0} \tilde{\sigma}_{s, d}^{2}}\right)^{\tau_{\mathrm{SD}}}(x)^{\tau_{\mathrm{SD}}-1} \exp \left(-\frac{x}{\mathbf{c}_{0} \tilde{\sigma}_{s, d}^{2}}\right)}{\Gamma\left(\tau_{\mathrm{SD}}\right)}, \\
& F_{\gamma_{\mathrm{SD}}(t)}(x)=\frac{\gamma\left(\tau_{\mathrm{SD}}, \frac{m_{\mathrm{SD}}}{\mathbf{c}_{0} \tilde{\sigma}_{S, d}^{2}} x\right)}{\Gamma\left(\tau_{\mathrm{SD}}\right)},
\end{aligned}
$$

$$
f_{\gamma_{\mathrm{SR}}^{(b)}(t)}(x)=\frac{\left(\frac{1}{\mathbf{c}_{2}\left(\tilde{\sigma}_{s, r}^{b}\right)^{2}}\right)^{\tau_{\mathrm{SR}}}(x)^{\tau_{\mathrm{SR}}-1} \exp \left(-\frac{x}{\mathbf{c}_{2}\left(\tilde{\sigma}_{\mathrm{S}, \mathrm{r}}^{b}\right)^{2}}\right)}{\Gamma\left(\tau_{\mathrm{SR}}\right)}
$$$$
F_{\gamma_{\mathrm{SR}}^{(b)}(t)}(x)=\frac{\gamma\left(\tau_{\mathrm{SR},} \frac{m_{\mathrm{SR}} x}{\mathbf{c}_{2}\left(\tilde{\sigma}_{S, r}^{b}\right)^{2}}\right)}{\Gamma\left(\tau_{\mathrm{SR}}\right)}
$$$$
f_{\gamma_{\mathrm{RD}}^{(b)}(t)}(x)=\frac{\left(\frac{1}{\mathbf{c}_{1}\left(\tilde{\sigma}_{r, d}^{b}\right)^{2}}\right)^{\tau_{\mathrm{RD}}}(x)^{\tau_{\mathrm{RD}}-1} \exp \left(-\frac{x}{\mathbf{c}_{1}\left(\tilde{\sigma}_{r, d}^{b}\right)^{2}}\right)}{\Gamma\left(\tau_{\mathrm{RD}}\right)}
$$$$
F_{\gamma_{\mathrm{RD}}^{(b)}(t)}(x)=\frac{\gamma\left(\tau_{\mathrm{RD}} \frac{m_{\mathrm{RD}} x}{\mathbf{c}_{1}\left(\tilde{\sigma}_{r, d}^{b}\right)^{b}}\right)}{\Gamma\left(\tau_{\mathrm{RD}}\right)} .
$$

The per-block average OP $\bar{P}_{\text {out }}\left(\gamma_{0}\right)$ at the destination node for multiple relay $\mathrm{DH}$ relaying network can be written as

$\left.\bar{P}_{\text {out }}\left(\gamma_{0}\right) \leq \frac{1}{M_{\mathrm{B}}} \sum_{t=1}^{M_{\mathrm{B}}} \sum_{j=0}^{L^{L-1}}\left\{\prod_{b \in \bar{\psi}_{j}^{t}}\left\{E_{\chi}\left\{P_{b}\left(\bar{\Phi}^{\prime}\right)\right\}\right\} \times \prod_{b \in \psi_{j}^{t}}\left\{1-E_{\chi}\left\{P_{b}\left(\bar{\Phi}^{\prime}\right)\right\}\right\} \times \prod_{b \in \psi_{j}^{t}} E_{\chi_{d}}\left\{P_{b}\left\{\left(\gamma_{\mathrm{SD}}(t)+\sum_{b \in \psi_{j}^{t}} \gamma_{\mathrm{RD}}^{(b)}(t)\right) \leq \gamma_{0}\right\}\right\}\right\}\right\}$. 
where

$E_{\chi}\left\{P_{b}\left(\bar{\Phi}^{\prime}\right)\right\}=E_{\chi}\left\{P_{b}\left(\gamma_{\mathrm{SR}}^{(b)}(t) \leq \gamma_{0}\right)\right\} \rightarrow$ Outage Probability of the event $\bar{\Phi}^{\prime}$ when $\gamma_{\mathrm{SR}}^{(b)}(t) \leq \gamma_{0}$

$E_{\chi}\left\{P_{b}\left(\Phi^{\prime}\right)\right\}=\left\{1-E_{\chi}\left\{P_{b}\left(\bar{\Phi}^{\prime}\right)\right\}\right\}=E_{\chi}\left\{P_{b}\left(\gamma_{\mathrm{SR}}^{(b)}(t) \geq \gamma_{0}\right)\right\} \rightarrow$ Outage Probability of the event $\bar{\Phi}^{\prime}$ when $\gamma_{\mathrm{SR}}^{(b)}(t) \geq \gamma_{0}$

$E_{\gamma_{d}}\left\{P_{b}\left\{\left(\gamma_{\mathrm{SD}}(t)+\sum_{b \in \psi_{j}^{t}} \gamma_{\mathrm{RD}}^{(b)}(t)\right) \leq \gamma_{0}\right\}\right\} \rightarrow$ Outage Probability of the cooperation mode.

The expression above can be written in the form of CDF

as

$\bar{P}_{\text {out }}\left(\gamma_{0}\right) \leq \frac{1}{M_{\mathrm{B}}} \sum_{t=1}^{M_{B}} \sum_{j=0}^{2^{L-1}}\left\{\prod_{b \in \bar{\psi}_{j}^{t}}\left\{F_{\gamma_{S R}^{(b)}(t)}\left(\gamma_{0}\right)\right\} \times \prod_{b \in \psi_{j}^{t}}\left\{1-F_{\gamma_{S R}^{(b)}(t)}\left(\gamma_{0}\right)\right\} \times\left\{F_{\mathbb{Z}_{d}(t)}\left(\gamma_{0}\right)\right\}\right\}$.

where $\mathbb{Z}_{d}(t)=\gamma_{\mathrm{SD}}(t)+\sum_{b \in \psi_{j}^{t}} \gamma_{\mathrm{RD}}^{(b)}(t)$ and $F_{\mathbb{Z}_{d}(t)}\left(\gamma_{0}\right)$ represents the $C D F$ of $\mathbb{Z}_{d}(t)$, expressed as

$F_{\mathbb{Z}_{d}(t)}\left(\gamma_{0}\right)=E_{\gamma_{d}}\left\{P_{b}\left\{\left(\gamma_{\mathrm{SD}}(t)+\sum_{b \in \psi_{j}^{t}} \gamma_{\mathrm{RD}}^{(b)}(t)\right) \leq \gamma_{0}\right\}\right\}$,

where $\gamma$ and $\gamma$ are expressed below

$$
\gamma=\left\{\gamma_{\mathrm{SR}}^{(1)}(t), \gamma_{\mathrm{SR}}^{(2)}(t), \ldots, \gamma_{\mathrm{SR}}^{(L)}(t)\right\}
$$

$\underline{\gamma}_{d}=\left\{\gamma_{\mathrm{SD}}(t), \gamma_{\mathrm{RD}}^{(1)}(t), \gamma_{\mathrm{RD}}^{(2)}(t), \ldots, \gamma_{\mathrm{RD}}^{(L)}(t)\right\}$.

Also $E_{\gamma}($.$) and E_{\gamma}$ (.) denote the expectation operator over the PDF of $\underline{\gamma}$ and $\underline{\gamma}_{d}$, respectively. The PDF of $\mathbb{Z}_{d}(t)$ can be derived from the characteristics function (CHF) of $\mathbb{Z}_{d}(t)$ which is the product of the CHF of $\gamma_{S D}(t)$ and $\sum_{b \in \psi_{j}^{t}} \gamma_{\mathrm{RD}}^{(b)}(t)$. The CHF of $\gamma_{S D}(t)$ is defined as

$\varphi_{\gamma_{\mathrm{SD}}(t)}(\omega)=\int_{0}^{\infty} f_{\gamma_{\mathrm{SD}}(t)}(x) e^{j \omega x} \mathrm{~d} x$.
Substituting $f_{\gamma_{\mathrm{SD}}(t)}(x)$ into (37) and using [(3.381.4), [50]], we get

$\varphi_{\gamma_{\mathrm{SD}}(t)}(\omega)=\left(1-j \omega \mathbf{c}_{0} \tilde{\sigma}_{s, d}^{2}\right)^{-\tau_{\mathrm{SD}}}$

Following a similar procedure, the $\mathrm{CHF}$ of $\gamma_{\mathrm{RD}}^{(b)}(t)$ is defined as

$\varphi_{\gamma_{\mathrm{RD}}^{(b)}(t)}(\omega)=\left(1-j \omega \mathbf{c}_{1}\left(\tilde{\sigma}_{r, d}^{b}\right)^{2}\right)^{-\tau_{\mathrm{RD}}}$,

The $\mathrm{CHF}$ of $\mathbb{Z}_{d}(t)$ is given as

$\varphi_{\mathbb{Z}_{d}(t)}(\omega)=\left(1-j \omega \mathbf{c}_{0} \tilde{\sigma}_{s, d}^{2}\right)^{-\tau_{\mathrm{SD}}} \prod_{b \in \psi_{j}^{t}}\left(1-j \omega \mathbf{c}_{1}\left(\tilde{\sigma}_{r, d}^{b}\right)^{2}\right)^{-\tau_{\mathrm{RD}}}$.

The pdf of $\mathbb{Z}_{d}(t)$ can be given by

$f_{\mathbb{Z}_{d}(t)}(x)=\frac{1}{2 \pi} \int_{0}^{\infty} \varphi_{\mathbb{Z}_{d}(t)}(\omega) e^{-i x \omega} \mathrm{d} \omega$

Substituting $\varphi_{\mathbb{Z}_{d}(t)}(\omega)$ in (41) and from [(3.384.7), [50]], we get

$f_{\mathbb{Z}_{d}(t)}(x)=\prod_{b \in \psi_{j}^{t}}\{\frac{e^{\left(-x / \mathbf{c}_{0} \tilde{\sigma}_{s, d}^{2}\right)} x^{\tau_{\mathrm{SD}}+\tau_{\mathrm{RD}}-1}\left(\mathbf{c}_{0} \tilde{\sigma}_{s, d}^{2}\right)^{-\tau_{\mathrm{SD}}}\left(\mathbf{c}_{1}\left(\tilde{\sigma}_{r, d}^{b}\right)^{2}\right)^{-\tau_{\mathrm{RD}}}}{\Gamma\left(\tau_{\mathrm{SD}}+\tau_{\mathrm{RD}}\right)} \underbrace{F_{1}\left(\tau_{\mathrm{SD}} ; \tau_{\mathrm{SD}}+\tau_{\mathrm{RD}} ;\left(\frac{1}{\mathbf{c}_{0} \tilde{\sigma}_{s, d}^{2}}-\frac{1}{\mathbf{c}_{1}\left(\tilde{\sigma}_{r, d}^{b}\right)^{2}}\right) x\right)}_{{ }_{1}(x)}\}$, 
where ${ }_{1} F_{1}($.$) is the confluent hypergeometric function of a$ single variable [(9.210), [50]], with [(9.211.2), [50]], we have

$g(x)=\frac{1}{\varpi\left(\tau_{\mathrm{SD}}, \tau_{\mathrm{RD}}\right)} \times \int_{0}^{1} e^{\left(\frac{1}{c_{0} \tilde{\sigma}_{S, d}^{2}}-\frac{1}{c_{1}\left(\sigma_{r, d}^{b} d^{2}\right.}\right) \times \Theta} \Theta^{\tau_{\mathrm{RD}}-1}(1-\Theta)^{\tau_{\mathrm{SD}}-1} \mathrm{~d} \Theta$,

where $\varpi\left(\tau_{\mathrm{SD}}, \tau_{\mathrm{RD}}\right)=\frac{\Gamma\left(\tau_{\mathrm{SD}}\right) \Gamma\left(\tau_{\mathrm{RD}}\right)}{\Gamma\left(\tau_{\mathrm{RD}}+\tau_{\mathrm{SD}}\right)}$ is beta function [50]. Substituting $g(x)$ in (42), we can express (42) as

$f_{\mathbb{Z}_{d}(t)}(x)=\prod_{b \in \psi_{j}^{t}}\left\{\tilde{\chi} e^{-x / \mathbf{c}_{0} \tilde{\sigma}_{s, d}^{2} X^{\tau_{\mathrm{SD}}+\tau_{\mathrm{RD}}-1}} \int_{0}^{1} e^{\left(\frac{1}{c_{0} \tilde{\sigma}_{s, d}^{2}}-\frac{1}{\boldsymbol{c}_{1}\left(\bar{\sigma}_{r, d}^{b}\right)^{2}}\right) x \Theta} \Theta^{\tau_{\mathrm{RD}}-1}(1-\Theta)^{\tau_{\mathrm{SD}}-1} d \Theta\right\}$,

where $\tilde{\chi} \triangleq \frac{\left(\mathbf{c}_{0} \tilde{\sigma}_{s, d}^{2}-\tau_{\mathrm{SD}}\left(\mathbf{c}_{1}\left(\tilde{\sigma}_{r, d}^{b}\right)^{2}\right)^{-\tau_{\mathrm{RD}}}\right.}{\Gamma\left(\tau_{\mathrm{SD}}+\tau_{\mathrm{RD}}\right) \varpi\left(\tau_{\mathrm{SD}}, \tau_{\mathrm{RD}}\right)}$. The CDF $F_{\mathbb{Z}_{d}(t)}\left(\gamma_{0}\right)$ can be expressed as

$$
\begin{aligned}
& F_{\mathbb{Z}_{d}(t)}\left(\gamma_{0}\right)=\int_{-\infty}^{\gamma_{0}} f_{\mathbb{Z}_{d}(t)}(x) \mathrm{d} x=1-\int_{\gamma_{0}}^{\infty} f_{\mathbb{Z}_{d}(t)}(x) \mathrm{d} x, \\
& =1-\int_{\gamma_{0}}^{\infty}\left\{\prod_{b \in \psi_{j}^{t}}\left\{\tilde{\chi} e^{-x / c_{0} \tilde{\sigma}_{s, d}^{2} \chi_{\mathrm{SD}}+\tau_{\mathrm{RD}}-1} \int_{0}^{1} e^{\left(\frac{1}{c_{0} \tilde{\sigma}_{s, d}^{2}}-\frac{1}{c_{1}\left(\tilde{\sigma}_{r, d}^{b}\right)^{2}}\right) x \Theta} \Theta^{\tau_{\mathrm{RD}}-1}(1-\Theta)^{\tau_{\mathrm{SD}}-1} \mathrm{~d} \Theta\right\}\right\} \mathrm{d} x, \\
& =1-\int_{\gamma_{0}}^{\infty}\left\{\tilde{\chi} e^{-\sum_{b \in \psi_{j}^{t}} \frac{1}{\sigma_{0}^{2} \tilde{\sigma}_{s, d}^{2}}\left(1-\left(1-\frac{c_{0} \tilde{\sigma}_{s, d}^{2}}{c_{1}\left(\tilde{\sigma}_{r, d}^{d} d^{2}\right.}\right) \Theta\right) x} x^{\tau_{\mathrm{SD}}+\tau_{\mathrm{RD}}-1} \int_{0}^{1} \Theta^{\tau_{\mathrm{RD}}-1}(1-\Theta)^{\tau_{\mathrm{SD}}-1} \mathrm{~d} \Theta\right\} \mathrm{d} x .
\end{aligned}
$$

After changing the order of integration in above expres-

sion, we get

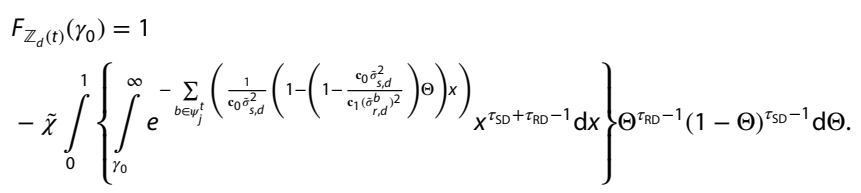

Using $\left.\tilde{l}=\int_{\gamma_{0}}^{\infty} e^{-\sum_{b \in \psi_{j}^{t}}\left(\frac{1}{c_{0} \tilde{\sigma}_{s, d}^{2}}\left(1-\left(1-\frac{c_{0} \tilde{\sigma}_{s, d}^{2}}{c_{1}\left(\tilde{\sigma}_{r, d}^{d}\right)^{2}}\right) \Theta\right) x\right.}\right)_{x^{\tau_{\mathrm{SD}}+\tau_{\mathrm{RD}}-1} \mathrm{~d} x,} \quad s=\frac{1}{\tilde{\sigma}_{s, d}^{2}}\left(1-\left(1-\frac{\mathbf{c}_{0} \tilde{\sigma}_{s, d}^{2}}{\mathbf{c}_{1}\left(\left(\tilde{\sigma}_{r, d}^{b}\right)^{2}\right.}\right) \Theta\right)$
$F_{\mathbb{Z}_{d}(t)}\left(\gamma_{0}\right)$ can be written as

$F_{\mathbb{Z}_{d}(t)}\left(\gamma_{0}\right)=P_{b}\left\{\mathbb{Z}_{d}(t) \leq \gamma_{0}\right\}=1-\tilde{\chi} \int_{0}^{1} \tilde{I} \Theta^{\tau_{\mathrm{RD}}-1}(1-\Theta)^{\tau_{\mathrm{SD}}-1} \mathrm{~d} \Theta$,

Using the series form of $\Gamma\left(\tau_{\mathrm{SD}}+\tau_{\mathrm{RD}}, s \gamma_{0}\right)$ [(8.352.2), [50]] (45)

$P_{b}\left\{\mathbb{Z}_{d}(t) \leq \gamma_{0}\right\}=1-\Gamma\left(\tau_{\mathrm{SD}}+\tau_{\mathrm{RD}}\right) \times \tilde{\chi} \underbrace{\int_{0}^{1}\left\{e^{-s \gamma_{0}} \sum_{m=0}^{\tau_{\mathrm{SD}}+\tau_{\mathrm{RD}}-1} \frac{\left(s \gamma_{0}\right)^{m}}{m !} s^{-\left(\tau_{\mathrm{SD}}+\tau_{\mathrm{RD}}\right)} \Theta^{\tau_{\mathrm{RD}}-1}(1-\Theta)^{\tau_{\mathrm{SD}}-1}\right\}}_{\rho^{\Theta}}\} \Theta$. 
It is significant to note that in the integral given above, the variable $s$ is a function of $\Theta$. Replacing $s$ in (48) by (47), the integral of (48), after some mathematical manipulations, can be expressed as

$$
\begin{aligned}
I^{\Theta} & =\sum_{m=0}^{\tau_{\mathrm{SD}}+\tau_{\mathrm{RD}}-1} \frac{\left(\gamma_{0}\right)^{m} e^{-\frac{\gamma_{0}}{c_{0} \tilde{\sigma}_{s, d}^{2}}}\left(\mathbf{c}_{0} \tilde{\sigma}_{s, d}^{2}\right)^{\tau_{\mathrm{SD}}+\tau_{\mathrm{RD}}-m}}{m !} \\
& \times \int_{0}^{1}\left\{(1-\Theta)^{\tau_{\mathrm{SD}}-1} \Theta^{\tau_{\mathrm{RD}}-1} e^{-\frac{\gamma_{0}}{c_{0} \tilde{\sigma}_{s, d}^{2}}\left(\frac{c_{0} \tilde{\sigma}_{s, d}^{2}}{\mathbf{c}_{1}\left(\tilde{\sigma}_{r, d}^{b}\right)^{2}}-1\right) \Theta}\right. \\
& \left.\times\left(1-\left(1-\frac{\mathbf{c}_{0} \tilde{\sigma}_{s, d}^{2}}{\mathbf{c}_{1}\left(\tilde{\sigma}_{r, d}^{b}\right)^{2}}\right) \Theta\right)^{-\left(\tau_{\mathrm{SD}}+\tau_{\mathrm{RD}}\right)+m}\right\} \mathrm{d} \Theta .
\end{aligned}
$$

Utilizing [(3.385), [50]], we can get the CF formulation for $l^{\Theta}$ in the form of the confluent hypergeometric function $\Phi_{1}\left\{A_{11}, B_{11}, C_{11}, \tilde{\alpha}, \tilde{\beta}\right\}$ of the variable $\tilde{\alpha}$ and $\tilde{\beta}[(9.261 .1)$, [50]] as

$$
\begin{aligned}
& P_{b}\left\{\mathbb{Z}_{d}(t) \leq \gamma_{0}\right\} \\
& =1-\frac{\left(\mathbf{c}_{0} \tilde{\sigma}_{s, d}^{2}\right)^{-\tau_{\mathrm{SD}}}\left(\mathbf{c}_{1}\left(\tilde{\sigma}_{r, d}^{b}\right)^{2}\right)^{-\tau_{\mathrm{RD}}}}{\varpi\left(\tau_{\mathrm{SD}}, \tau_{\mathrm{RD}}\right)} \\
& \sum_{m=0}^{\tau_{\mathrm{SD}}+\tau_{\mathrm{RD}}-1} \frac{\left(\gamma_{0}\right)^{m} e^{-\frac{\gamma_{0}^{0}}{\tilde{s}_{s, d}}\left(\mathbf{c}_{0} \tilde{\sigma}_{s, d}^{2}\right)^{\left(\tau_{\mathrm{SD}}+\tau_{\mathrm{RD}}\right)-m}}}{m !} \\
& \Phi_{1}\left(\tau_{\mathrm{RD}}, \tau_{\mathrm{SD}}+\tau_{\mathrm{RD}}-m, \tau_{\mathrm{SD}}+\tau_{\mathrm{RD}}\right. \\
& \left.-\left(\frac{1}{\mathbf{c}_{1}\left(\tilde{\sigma}_{r, d}^{b}\right)^{2}}-1\right) \gamma_{0}\left(1-\frac{\mathbf{c}_{0} \tilde{\sigma}_{s, d}^{2}}{\mathbf{c}_{1}\left(\tilde{\sigma}_{r, d}^{b}\right)^{2}}\right)\right) .
\end{aligned}
$$

For ${ }_{x} F_{y}($.$) , routine is available in Maple or MATLAB, but no$ routine is available for computing the confluent hypergeometric function $\Phi_{1}\left\{A_{11}, B_{11}, C_{11}, \tilde{\alpha}, \tilde{\beta}\right\}$ of the variable $\tilde{\alpha}$ and $\tilde{\beta}$. However, the series $\Phi_{1}\{$.$\} is computed proficiently as it$ will converge after a limited number of terms because $\left(1-\frac{\mathbf{c}_{0}}{\mathbf{c}_{1}}\right)<1$. Substituting (51) into (36), the per-block average OP can be expressed as

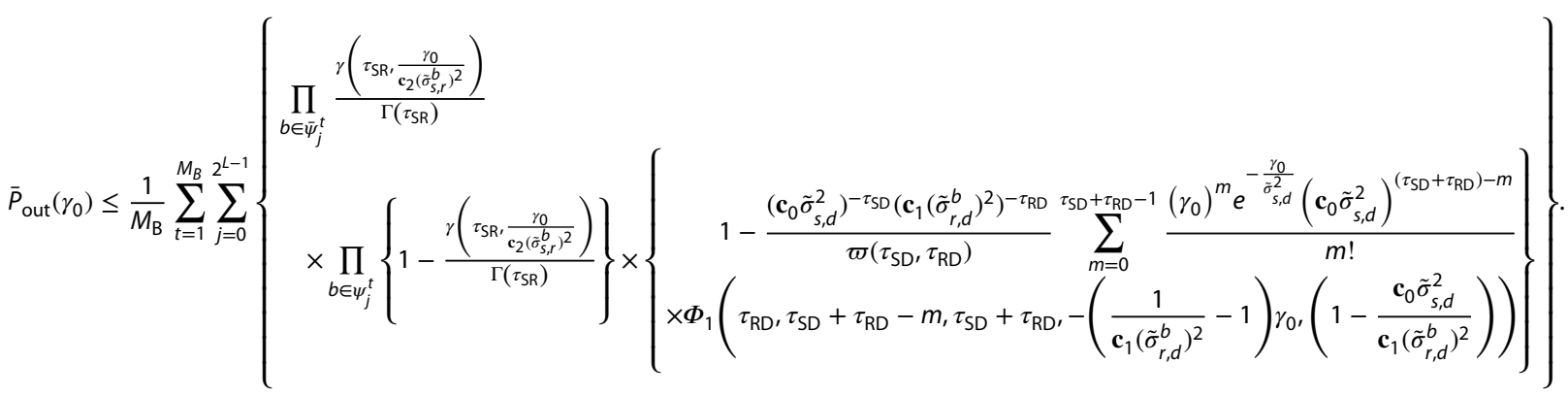

$$
\begin{aligned}
& I^{\Theta}=\sum_{m=0}^{\tau_{\mathrm{SD}}+\tau_{\mathrm{RD}}-1} \frac{\left(\gamma_{0}\right)^{m} e^{-\frac{\gamma_{0}}{c_{0} \tilde{\sigma}_{s, d}^{2}}}\left(\mathbf{c}_{0} \tilde{\sigma}_{s, d}^{2}\right)^{\left(\tau_{\mathrm{SD}}+\tau_{\mathrm{RD}}\right)-m}}{m !} \\
& \times \Phi_{1}\left(\tau_{\mathrm{RD}}, \tau_{\mathrm{SD}}+\tau_{\mathrm{RD}}-m, \tau_{\mathrm{SD}}+\tau_{\mathrm{RD}},\right. \\
&\left.-\left(\frac{1}{\mathbf{c}_{1}\left(\tilde{\sigma}_{r, d}^{b}\right)^{2}}-1\right) \gamma_{0},\left(1-\frac{\mathbf{c}_{0} \tilde{\sigma}_{s, d}^{2}}{\mathbf{c}_{1}\left(\tilde{\sigma}_{r, d}^{b}\right)^{2}}\right)\right) .
\end{aligned}
$$

Substituting (50) into (48), $P_{b}\left\{\mathbb{Z}_{d}(t) \leq \gamma_{0}\right\}$ can be expressed as

\subsection{DO and optimal power allocation analysis}

1. DO analysis

For high values of $P_{S} / N_{0}$ and $P_{b} / N_{0}, \bar{P}_{\text {out }}\left(\gamma_{0}\right) \rightarrow 0$. $\bar{P}_{\text {out }}\left(\gamma_{0}\right) \rightarrow 0$ can be easily expressed by using ${ }^{\Theta}$ for $\Phi_{1}($. and using expressions [(3.197.3), [50]], [(8.352.1), [50]], [(9.131), [50]], [(8.352.1), [50]], and [(8.352.2), [50]]. Also, it can be easily verified from (52) that for extremely low values of SNR, that is, $P_{S} / N_{0} \rightarrow 0$ and $P_{b} / N_{0} \rightarrow 0, \bar{P}_{\text {out }}\left(\gamma_{0}\right) \rightarrow 1$. Further, the $\mathrm{DO}$ analysis can be further simplified by using $\mathbf{c}_{0}=\mathbf{c}_{1}, \Phi_{1}$ (.) in (51) approaches to unity. After applying [(8.352.1), [50]] into (51), (52) can be simplified as

$$
\bar{P}_{\text {out }}\left(\gamma_{0}\right) \leq \frac{\gamma\left(\tau_{\mathrm{SD}}, \frac{m_{\mathrm{SS}} \gamma_{0}}{\mathrm{c}_{0} \tilde{\sigma}_{S, d}^{2}}\right)}{\Gamma\left(\tau_{\mathrm{SD}}\right)} \times \frac{\gamma\left(\tau_{\mathrm{SR}}, \frac{m_{\mathrm{SR}} \gamma_{0}}{\mathrm{c}_{2}\left(\tilde{\sigma}_{S, r}^{b}\right)^{2}}\right)}{\Gamma\left(\tau_{\mathrm{SR}}\right)}+\frac{\gamma\left(\tau_{\mathrm{SD}}+\tau_{\mathrm{RD}}, \frac{m_{\mathrm{S}} \gamma_{0}}{\mathrm{c}_{0} \tilde{\sigma}_{s, d}^{2}}\right)}{\Gamma\left(\tau_{\mathrm{SD}}+\tau_{\mathrm{RD}}\right)} \times \frac{\Gamma\left(\tau_{\mathrm{SR}}, \frac{m_{\mathrm{SR}} \gamma_{0}}{c_{2}\left(\tilde{\sigma}_{S, r}^{b}\right)^{2}}\right)}{\Gamma\left(\tau_{\mathrm{SR}}\right)}
$$

For higher SNR regimes, the relaying significantly improves the DO. For $P_{\mathrm{S}} / N_{0} \rightarrow 0$ and $P_{b} / N_{0} \rightarrow 0$, the expression given in (53) can be expressed as 
$\bar{P}_{\text {out }}\left(\gamma_{0}\right) \leq \frac{\gamma_{0}^{\tau_{\mathrm{SD}}+\tau_{\mathrm{SR}}}\left(N_{0} / P\right)^{-\left(\tau_{\mathrm{SD}}+\tau_{\mathrm{SR}}\right)}}{\mathbf{c}_{0}^{\tau_{\mathrm{SD}}} \mathbf{c}_{2}^{\tau_{\mathrm{SR}}} \Gamma\left(\tau_{\mathrm{SD}}+1\right) \Gamma\left(\tau_{\mathrm{SR}}+1\right)}+\frac{\gamma_{0}^{\tau_{\mathrm{SD}}+\tau_{\mathrm{SR}}}\left(N_{0} / P\right)^{-\left(\tau_{\mathrm{SD}}+\tau_{\mathrm{SR}}\right)}}{2 \tau_{\mathrm{SD}} \mathbf{c}_{0}^{\tau_{\mathrm{SD}}+\tau_{\mathrm{SR}}} \Gamma\left(\tau_{\mathrm{SD}}+\tau_{\mathrm{SR}}\right)}-\frac{\gamma_{0}^{\tau_{\mathrm{SD}}+\tau_{\mathrm{SR}}+\tau_{\mathrm{RD}}}\left(N_{0} / P\right)^{-\left(\tau_{\mathrm{SD}}+\tau_{\mathrm{SR}}+\tau_{\mathrm{RD}}\right)}}{2\left(\tau_{\mathrm{SD}}+\tau_{\mathrm{SR}}\right) \mathbf{c}_{0}^{\tau_{\mathrm{SD}}+\tau_{\mathrm{SR}}} \mathbf{c}_{2}^{\tau_{\mathrm{SR}}} \Gamma\left(\tau_{\mathrm{SD}}+1\right) \Gamma\left(\tau_{\mathrm{SR}}\right)}$

It is clear from the expression of $\bar{P}_{\text {out }}\left(\gamma_{0}\right)$ that first two terms are dominant terms. The $\mathrm{DO}$ is equal to $\tau_{\mathrm{SD}}+\tau_{\mathrm{SR}}$ and $2 \tau_{\mathrm{SR}}$ for $\left(\left(m_{\mathrm{RD}}\right) \times(\text { linkstrength })_{\mathrm{RD}}\right)>\left(\left(m_{\mathrm{SR}}\right) \times(\text { linkstrength })_{\mathrm{SR}}\right)$ and $\left.\left(m_{\mathrm{RD}}\right) \times(\text { linkstrength })_{\mathrm{RD}}\right)<\left(\left(m_{\mathrm{SR}}\right) \times(\text { linkstrength })_{\mathrm{SR}}\right)$, respectively. Also, for the case when instantaneous SNR at relay node is greater than the threshold SNR, the asymptotic OP is fully defined by the second term of (54), and thus, the DO is equal to $2 \tau_{\mathrm{SD}}$ regardless of the values of $M_{\mathrm{D}}$ and $M$.

\section{OP allocation analysis}

Incase of $\left(\left(m_{\mathrm{RD}}\right) \times(\text { linkstrength })_{\mathrm{RD}}\right)>\left(\left(m_{\mathrm{SR}}\right) \times(\text { linkstrength })_{\mathrm{SR}}\right)$, the term corresponding to $P_{S} / P$ dominates in the PEP's expression; it means that we have to maximize the value of $P_{\mathrm{S}} / P$ to minimize the value of the PEP. As a result, almost all the power is allocated to the source node only for getting better decoding at the relay node. Also, in this scenario, the maximum value of $P_{\mathrm{S}} / P$ is equal to unity. Further, $\left(P_{S} / P\right) \Pi_{b=1}^{L}\left(P_{b} / P\right) ; 1 \leq b \leq L$ dominates in the PEP error-bound expression for $\left(\left(m_{\mathrm{RD}}\right) \times(\text { linkstrength })_{\mathrm{RD}}\right)<\left(\left(m_{\mathrm{SR}}\right) \times(\text { linkstrength })_{\mathrm{SR}}\right), \quad$. After solving the $C O$ problem, we get $P_{\mathrm{S}} / P=P_{b} / P=1 /(L+1) ; 1 \leq b \leq L$, i.e., equal power allocation is the best possible outcome in this scenario. When $\left(m_{\mathrm{RD}} \times\right.$ linkstrength $\left._{\mathrm{RD}}\right)=\left(m_{\mathrm{SR}} \times\right.$ linkstrength $\left._{\mathrm{SR}}\right)$, we can compute the $\mathrm{CO}$ problem as

$\underbrace{\min }_{P_{\mathrm{S}} / P_{,} / P \ldots P_{\mathrm{L}} / P} \sum_{j=0}^{2^{L-1}}\left\{K^{\prime}\left(\frac{P}{P_{\mathrm{S}}}\right)^{M M_{\mathrm{D}}+M^{2}\left|\bar{\psi}_{j}\right|}\left(\frac{P}{P_{b}}\right)^{M M_{\mathrm{D}}\left|\psi_{j}\right|}\right\}$

s.t. $P_{\mathrm{S}} / P+\sum_{b=1}^{L}\left(P_{b} / P\right)=1$,
$\left(P_{\mathrm{S}} / P\right) \geq 0$,

$\left(P_{b} / P\right) \geq 0,1 \leq b \leq L$.

where $K^{\prime}$ is given below

$K^{\prime}=\left\{\prod_{b \in \bar{\psi}_{j}} T_{1}\right\} \times\left\{\prod_{b \in \psi_{j}} T_{2}\right\}\left(\frac{N_{0}}{P}\right)^{\tau_{\mathrm{SD}}+\tau_{\mathrm{RD}}\left|\psi_{j}\right|+\tau_{\mathrm{SR}}\left|\bar{\psi}_{j}\right|}$.

The above $\mathrm{CO}$ [51] problem is a geometric progression (GP) [52], and it can be solved by using MATLAB software for disciplined convex programming (CVX SOLVER) [53].

\subsection{Asymptotic error floor analysis}

1. Nodes' mobility effect

As an impact of mobility of nodes and imperfect CSI estimation, the PEP performance of the system experiences degradation, especially for high values of $P_{\mathrm{S}} / N_{0}$ and $P_{b} / N_{0}$. This degradation is mainly represented by irreducible conditional PEP error floors, which can be expressed as

$\bar{P}_{\mathrm{E}}^{F}=\lim _{\substack{P_{\mathrm{s}} / N_{0 \prime} \\ P_{b} / N_{0} \rightarrow \infty}} \bar{P}_{\mathrm{E}}$

One can derive the asymptotic error floor limit for $\mathrm{DH}$ $S$-DF relaying protocol by ignoring the additive unity terms from the denominator of $\gamma_{\mathrm{SD}}(t), \gamma_{\mathrm{SR}}^{(b)}(t)$ and $\gamma_{\mathrm{RD}}^{(b)}(t)$ for high values of $P_{\mathrm{S}} / N_{0}$ and $P_{b} / N_{0}$ can be written in a simplified form as

$\bar{\gamma}_{\mathrm{SD}}^{a v}=\rho_{\mathrm{SD}}^{2(t-1)}\left\{\rho_{\mathrm{SD}}^{2(t-1)} \tilde{\sigma}_{\in \mathrm{SD}}^{2}+\left(1-\rho_{\mathrm{SD}}^{2(t-1)}\right) \tilde{\sigma}_{\mathrm{eSD}}^{2}\right\}^{-1}$,

$\tilde{\mathbb{N}}_{\text {eff }}^{i}=\left(N_{0}+\left(\rho_{i}^{(b)}\right)^{2(t-1)} \tilde{\sigma}_{\epsilon_{i}}^{2}+\left(1-\left(\rho_{i}^{(b)}\right)^{2(t-1)}\right) \tilde{\sigma}_{e_{i}}^{2}\right), \quad i \in(\mathrm{SR}, \mathrm{RD})$

Also the effective noise variance $\tilde{\mathbb{N}}_{\text {eff }}^{i}$ and $\tilde{\mathbb{N}}_{\text {eff }}^{S D}$ can be represented as [16-18], 
$\tilde{\mathbb{N}}_{\text {eff }}^{S \mathrm{D}}=\left(N_{0}+\rho_{\mathrm{SD}}^{2(t-1)} \sigma_{\in \mathrm{SD}}^{2}+\left(1-\rho_{\mathrm{SD}}^{2(t-1)}\right) \sigma_{e S \mathrm{D}}^{2}\right)$.

Substituting (61) and (62) into (34), the per-block average asymptotic error floor can be expressed as
For the network scenario, when $\rho_{\mathrm{SR}}^{(b)}=\rho_{\mathrm{SD}}=\rho_{\mathrm{RD}}^{(b)}=1$ and $\sigma_{\in \mathrm{SD}}^{2}=\sigma_{\in \mathrm{SR}}^{2}=\sigma_{\in \mathrm{RD}}^{2}=0$, in this case, the asymptotic limits for $\bar{P}_{\mathrm{E}}^{F}$ is still given by (63), but with the following modified parameters $\tilde{\mathbb{N}}_{\text {eff' }}^{R D}=\sigma_{\in R D^{\prime}}^{2} \tilde{\mathbb{N}}_{\text {eff }}^{S R}=\sigma_{\in S R}^{2}$ and $\tilde{\mathbb{N}}_{\text {eff }}^{S D}=\sigma_{\in S D}^{2}[44]$.

$\bar{P}_{\mathrm{E}}^{F} \leq \frac{1}{M_{\mathrm{B}}} \sum_{t=1}^{M_{\mathrm{B}}} \sum_{i=1, s \neq i}^{|\mathrm{Y}|} \sum_{j=0}^{2^{L-1}}\left\{\prod_{b \in \bar{\psi}_{j}^{t}}\left\{\sum_{l=1}^{|\mathrm{Y}|}\left(\tilde{\Xi}_{1}-\tilde{\Xi}_{2}\right)\right\} \times \prod_{b \in \psi_{j}^{t}}\left\{1-\sum_{l=1}^{|\mathrm{Y}|}\left(\tilde{\Xi}_{1}-\tilde{\Xi}_{2}\right)\right\} \times\left(\left(\tilde{\widetilde{\sigma}}_{1}-\tilde{\widetilde{\sigma}}_{2}\right) \prod_{b \in \psi_{j}^{t}}\left(\tilde{\mathrm{O}}_{1}-\tilde{\mathrm{O}}_{2}\right)\right)\right\}$.

where $\tilde{\Xi}_{1}-\tilde{\Xi}_{2}, \tilde{\mho}_{1}-\tilde{\mho}_{2}$ and $\tilde{O}_{1}-\tilde{O}_{2}$ are expressed in (64).

$$
\begin{aligned}
& \tilde{\Xi}_{1}-\tilde{\Xi}_{2}=\frac{a^{\prime}}{2 \pi}\left(\frac{4 N_{a} \tilde{\mathbb{N}}_{\mathrm{eff}}^{S R}}{4 N_{a} \tilde{\mathbb{N}}_{\mathrm{eff}}^{\mathrm{SR}}+b^{\prime}\left(\rho_{\mathrm{SR}}^{(b)}\right)^{2(t-1)} \lambda_{\mathrm{ln}}^{2} \mathbf{c}_{2}\left(\tilde{\sigma}_{S, r}^{b}\right)^{2}}\right)^{\tau_{\mathrm{SR}}} \frac{\Gamma\left(\tau_{\mathrm{SR}}\right)}{\Gamma\left(\tau_{\mathrm{SR}}+1\right)^{2}} F_{1}\left(\tau_{\mathrm{SR}}, \frac{1}{2} ; \tau_{\mathrm{SR}}+1 ; \frac{1}{1+\frac{b^{\prime}\left(\rho_{\mathrm{SR}}^{(b)}\right)^{2(t-1)} \lambda_{\mathrm{ln}}^{2} \mathrm{c}_{2}\left(\tilde{\sigma}_{S, r}^{b}\right)^{2}}{4 N_{a} \tilde{\mathbb{N}}_{\mathrm{SRf}}}}\right) \\
& -\frac{c^{\prime}}{\pi}\left(\frac{4 N_{a} \tilde{\mathbb{N}}_{\text {eff }}^{S R}}{4 N_{a} \tilde{\mathbb{N}}_{\text {eff }}^{S R}+b^{\prime}\left(\rho_{\mathrm{SR}}^{(b)}\right)^{2(t-1)} \lambda_{\ln }^{2} \mathbf{c}_{2}\left(\tilde{\sigma}_{s, r}^{b}\right)^{2}}\right)^{\tau_{\mathrm{SR}}} \frac{\Gamma\left(\tau_{\mathrm{SR}}+\frac{1}{2}\right)}{\Gamma\left(\tau_{\mathrm{SR}}+\frac{3}{2}\right)} \mathbf{F}_{\mathrm{A}}^{(1)}\left(\tau_{\mathrm{SR}}+\frac{1}{2} ; \tau_{\mathrm{SR}} ; \frac{1}{2} ; \tau_{\mathrm{SR}}+\frac{3}{2} ; \frac{2 b^{\prime}\left(\rho_{\mathrm{SR}}^{(b)}\right)^{2(t-1)} \lambda_{\mathrm{ln}}^{2} \mathbf{c}_{2}\left(\tilde{\sigma}_{s, r}^{b}\right)^{2}}{4 N_{a} \tilde{\mathbb{N}}_{\mathrm{eff}}^{\mathrm{SR}}} ; \frac{1}{2}\right),
\end{aligned}
$$

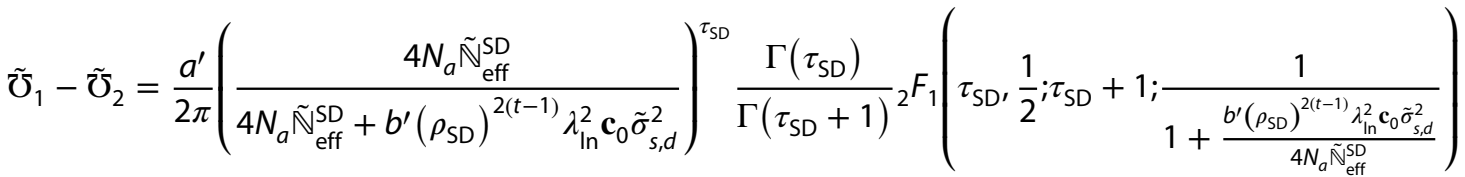

$$
\begin{aligned}
& -\frac{c^{\prime}}{\pi}\left(\frac{4 N_{a} \tilde{\mathbb{N}}_{\text {eff }}^{\mathrm{SD}}}{4 N_{a} \tilde{\mathbb{N}}_{\text {eff }}^{\mathrm{SD}}+b^{\prime}\left(\rho_{\mathrm{SD}}\right)^{2(t-1)} \lambda_{\mathrm{In}}^{2} \mathbf{c}_{0} \tilde{\sigma}_{s, d}^{2}}\right)^{\tau_{\mathrm{SD}}} \frac{\Gamma\left(\tau_{\mathrm{SD}}+\frac{1}{2}\right)}{\Gamma\left(\tau_{\mathrm{SD}}+\frac{3}{2}\right)} \mathbf{F}_{\mathrm{A}}^{(1)}\left(\tau_{\mathrm{SD}}+\frac{1}{2} ; \tau_{\mathrm{SD}}, 1 / 2 ; \tau_{\mathrm{SD}}+\frac{3}{2} ; \frac{2 b^{\prime}\left(\rho_{\mathrm{SD}}\right)^{2(t-1)} \lambda_{\mathrm{ln}}^{2} \mathbf{c}_{0} \tilde{\sigma}_{s, d}^{2}}{4 N_{a} \tilde{\mathbb{N}}_{\mathrm{eff}}^{\mathrm{SD}}} ; 1 / 2\right),
\end{aligned}
$$

$$
\begin{aligned}
& \tilde{\mathrm{O}}_{1}-\tilde{\mathrm{O}}_{2}= \\
& \frac{a^{\prime}}{2 \pi}\left(\frac{4 N_{a} \tilde{\mathbb{N}}_{\mathrm{eff}}^{\mathrm{RD}}}{4 N_{a} \tilde{\mathbb{N}}_{\mathrm{eff}}^{\mathrm{RD}}+b\left(\rho_{\mathrm{RD}}^{(b)}\right)^{2(t-1)} \lambda_{\ln }^{2} \mathbf{c}_{1}\left(\tilde{\sigma}_{r, d}^{b}\right)^{2}}\right)^{\tau_{\mathrm{RD}}} \frac{\Gamma\left(\tau_{\mathrm{RD}}\right)}{\Gamma\left(\tau_{\mathrm{RD}}+1\right)}{ }_{2} F_{1}\left(\tau_{\mathrm{RD}}, \frac{1}{2} ; \tau_{\mathrm{RD}}+1 ; \frac{1}{\left.1+\frac{b^{\prime}\left(\rho_{\mathrm{RD}}^{(b)}\right)^{2(t-1)} \lambda_{\mathrm{ln}}^{2} \mathbf{c}_{1}\left(\tilde{\sigma}_{r, d}^{b}\right)^{2}}{4 N_{a} \tilde{\mathbb{N}}_{\mathrm{eff}}}\right)}\right. \\
& -\frac{c^{\prime}}{\pi}\left(\frac{4 N_{a} \tilde{\mathbb{N}}_{\mathrm{eff}}^{\mathrm{RD}}}{4 N_{a} \tilde{\mathbb{N}}_{\mathrm{eff}}^{\mathrm{RD}}+b^{\prime}\left(\rho_{\mathrm{RD}}^{(b)}\right)^{2(t-1)} \lambda_{\mathrm{ln}}^{2} \mathbf{c}_{1}\left(\tilde{\sigma}_{r, d}^{b}\right)^{2}}\right)^{\tau_{\mathrm{RD}}} \frac{\Gamma\left(\tau_{\mathrm{RD}}+\frac{1}{2}\right)}{\Gamma\left(\tau_{\mathrm{RD}}+\frac{3}{2}\right)} \mathbf{F}_{\mathrm{A}}^{(1)}\left(\tau_{\mathrm{RD}}+\frac{1}{2} ; \tau_{\mathrm{RD}}, \frac{1}{2} ; \tau_{\mathrm{RD}}+\frac{3}{2} ; \frac{2 b^{\prime}\left(\rho_{\mathrm{RD}}^{(b)}\right)^{2(t-1)} \lambda_{\mathrm{ln}}^{2} \mathbf{c}_{1}\left(\tilde{\sigma}_{r, d}^{b}\right)^{2}}{4 N_{a} \tilde{\mathbb{N}}_{\mathrm{eff}}^{S R}} ; \frac{1}{2}\right) .
\end{aligned}
$$

It is worth mentioning that when all of the nodes are static and the estimation processes are perfect, the floor in (63), as expected, vanishes, i.e., nodes' mobility impact is removed.

2. Various conditions arise due to nodes' mobility a. For static nodes and perfect estimation of channel
Furthermore, for perfect CSI, the value of $\tilde{\mathbb{N}}_{\text {eff }}^{S D} \tilde{\mathbb{N}}_{\text {eff }}^{S R}$ and $\tilde{\mathbb{N}}_{\text {eff }}^{R D}$ equals to zero which reduces (63) to zero, i.e., impact of node mobility is removed.

b. When relay nodes are mobile and source and destination nodes are static 


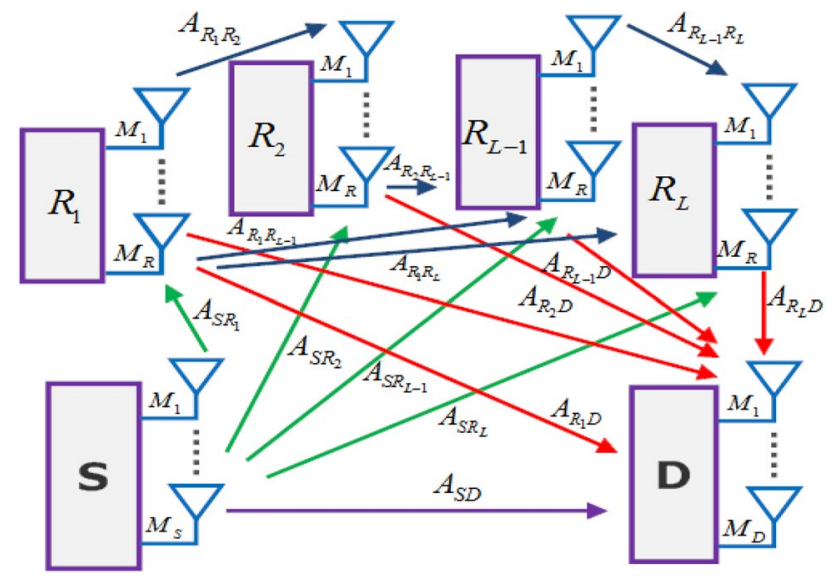

Fig. 4 Schematic diagram of multiple hop $(\mathrm{MH})$, multiple phase, multiple relay $W(\mathrm{~m})$ cooperation protocol $[38,39]$

Corollary 1 For the network scenario, when $\rho_{\mathrm{SR}}^{(b)}<1, \rho_{\mathrm{SD}}=1, \rho_{\mathrm{RD}}^{(b)}<1$ and $\sigma_{\in \mathrm{SD}}^{2}=\sigma_{\in \mathrm{SR}}^{2}=\sigma_{\in \mathrm{RD}}^{2}=0$, the system performance does not experience asymptotic limits if $\rho_{\mathrm{SD}}=1$ that is both destination and source nodes are static.

Proof For this network scenario, the asymptotic error floor is still computed from expression given in (63) with the following modified parameters: $\tilde{\mathbb{N}}_{\text {eff }}^{\mathrm{SD}}=\sigma_{\in \mathrm{SD}}^{2}, \tilde{\mathbb{N}}_{\text {eff }}^{\mathrm{RD}}=\left(\rho_{\mathrm{RD}}^{(b)}\right)^{2(t-1)} \sigma_{\in \mathrm{RD}}^{2}+\left(1-\left(\rho_{\mathrm{RD}}^{(b)}\right)^{2(t-1)}\right) \sigma_{\mathrm{eRD}}^{2} \quad$ a n d $\tilde{\mathbb{N}}_{\text {eff }}^{S R}=\left(\rho_{\mathrm{SR}}^{(b)}\right)^{2(t-1)} \sigma_{\in \mathrm{SR}}^{2}+\left(1-\left(\rho_{\mathrm{SR}}^{(b)}\right)^{2(t-1)}\right) \sigma_{\mathrm{eSR}}^{2}$. Furthermore, for perfect channel estimation, the value of $\tilde{\mathbb{N}}_{\text {eff }}^{S D}, \tilde{\mathbb{N}}_{\text {eff }}^{R D}$ and $\tilde{\mathbb{N}}_{\text {eff }}^{S R} \approx 0$ and it yields $\bar{P}_{\mathrm{E}}^{F}=0$, i.e., fading links are no longer time selective. Although in the case of perfect CSI, both scenarios 2(a) and 2(b) do not provide asymptotic limits, this does not mean that they provide the same value over all the non-infinite SNR range. Later in the simulation results section, we can demonstrate that due to relay mobility, scenario 2(b) provides worse performance than scenario 2(a).

c. When relay and source nodes are not moving and destination node is mobile

Corollary 2 For DH relaying networks, while the relays are static, the system performance is severely degraded by asymptotic limits as long as the destination node is in motion.

Proof When $\rho_{\mathrm{SR}}^{(b)}=1, \rho_{\mathrm{SD}}<1$ and $\rho_{\mathrm{RD}}^{(b)}<1$. In this case, the asymptotic limit is given in (63), but with the following modified parameters: $\tilde{\mathbb{N}}_{\text {eff }}^{S R}=\sigma_{\in S R^{\prime}}^{2}, \tilde{\mathbb{N}}_{\text {eff }}^{S D}=\rho_{\mathrm{SD}}^{2(t-1)} \sigma_{\epsilon_{\mathrm{SD}}}^{2}+\left(1-\rho_{\mathrm{SD}}^{2(t-1)}\right) \sigma_{\mathrm{SDD}_{\mathrm{D}}}^{2} \quad$ a $\mathrm{n} \mathrm{d}$ $\tilde{\mathbb{N}}_{\text {eff }}^{R D}=\left(\rho_{\mathrm{RD}}^{(b)}\right)^{2(t-1)} \sigma_{\in \mathrm{RD}}^{2}+\left(1-\left(\rho_{\mathrm{RD}}^{(b)}\right)^{2(t-1)}\right) \sigma_{e R D}^{2}$. It can be easily seen that when $b$ th relay decodes the OSTBC code word successfully, the PEP term for the case, i.e., $\left\{1-\bar{P}_{\mathrm{E}}^{S R}\left(B_{s}(t) \rightarrow B_{l}(t)\right)\right\}$ in (63), contributes to the asymp-

Probability when relay

decodes correctly

totic error floor for the per-block average PEP.

d. When relay and destination nodes are static and source node is mobile

Corollary 3 In DH multiple relay S-DF cooperative communication networks, even though relay is static, the system performance is severely degraded by asymptotic limits if the source node is in motion.

Proof When $\rho_{\mathrm{RD}}^{(b)}=1$ while $\rho_{\mathrm{SD}}$ and $\rho_{\mathrm{SR}}^{(b)}$ are $<1$. The asymptotic limit is given by (63), but with the following modified parameters: $\tilde{\mathbb{N}}_{\text {eff }}^{R D}=\sigma_{\in R D}^{2}, \tilde{n}_{\mathrm{SD}}=\rho_{\mathrm{SD}}^{2(t-1)} \sigma_{\in \mathrm{SD}}^{2}+\left(1-\rho_{\mathrm{SD}}^{2(t-1)}\right) \sigma_{\mathrm{eSD}}^{2}$ and $\tilde{\mathbb{N}}_{\text {eff }}^{\mathrm{SR}}=\left(\rho_{\mathrm{SR}}^{(b)}\right)^{2(\tau-1)} \sigma_{\in \mathrm{SR}}^{2}+\left(1-\left(\rho_{\mathrm{SR}}^{(b)}\right)^{2(\tau-1)}\right) \sigma_{\mathrm{eSR}}^{2}$. In this scenario, it is significant to note that the PEP term for the event when $b$ th relay decodes the MIMO STBC code word incorrectly, i.e., $1-\bar{P}_{\mathrm{E}}^{\mathrm{SR}}\left(B_{s}(t) \rightarrow B_{l}(t)\right)$ in (63) contributes to Error Probability when relay decodes incorrectly

the asymptotic error floor for the per-block average PEP.

\subsection{Asymptotic PEP analysis of DH cooperative communication protocol}

In this subsection, we demonstrate the DO analysis and will develop a framework to evaluate the optimal source-relay power allocation factors, which will further enhance the end-to-end error performance of the relaying network. We consider that each receiving terminal has perfect channel estimation, i.e., $\sigma_{\in \mathrm{SD}}^{2}=\sigma_{\in \mathrm{SR}}^{2}=\sigma_{\in \mathrm{RD}}^{2}=0$ and nodes are static, i.e., $\sigma_{\mathrm{eSD}}^{2}=\sigma_{\mathrm{eSR}}^{2}=\sigma_{\mathrm{eRD}}^{2}=0$. Applying the above-mentioned conditions in (33) and at high SNR approximating $\prod_{b \in \psi_{j}^{t}}\left\{1-\sum_{l=1}^{|\Upsilon|}\left(\Xi_{1}-\Xi_{2}\right)\right\}$ and considering the dominating terms corresponding to $m, n=0,1$ in the identity ${ }_{2} F_{1}(a, b ; c ; z)=\sum_{n=0}^{K} \frac{(a)_{n}(b)_{n} z^{n}}{(c)_{n} n !}$ for the Gauss hypergeometric function and Appell hypergeometric function of two variables [54],

$\mathrm{F}_{\mathrm{A}}^{(1)}\left(\chi_{1}, l_{1}, l_{1}^{\prime}, \gamma^{\prime \prime} ; x^{\prime}, y^{\prime}\right)=\sum_{m, n=0}^{\infty} \frac{\left(\chi_{1}\right)_{m+n}\left(l_{1}\right)_{m}\left(l_{1}^{\prime}\right)_{n}}{\left(\gamma^{\prime \prime}\right)_{m+n} \Gamma(m+1) \Gamma(n+1)}\left(x^{\prime}\right)^{m}\left(y^{\prime}\right)^{n}$, 
where $(x)_{n}$ is the Pochhammer symbols [54], one can sim-

and $W(1)$ is the least complex $\mathrm{MH}$ multiple phase relaying plify the expression in (33), as given in (65).

protocol [30]. The schematic representation of $\mathrm{MH}$ multi-

$P^{\mathrm{Asy}}\left(B_{s}(t) \rightarrow B_{i}(t)\right)=\sum_{j=0}^{2^{L-1}}\left\{\left\{\prod_{b \in \bar{\psi}_{j}^{t}} T_{1}\left(\frac{1}{\beta_{0}}\right)^{\tau_{\mathrm{SD}}+\tau_{\mathrm{SR}}\left|\bar{\psi}_{j}^{t}\right|}\left(\frac{1}{\beta_{0}}\right)^{\tau_{\mathrm{SD}}}\right\} \times\left\{\prod_{b \in \psi_{j}^{t}} \mathrm{~T}_{2}\left(\frac{1}{\beta_{b}}\right)^{\tau_{\mathrm{RD}}\left|\psi_{j}^{t}\right|}\right\}\left(\frac{N_{0}}{P}\right)^{\tau_{\mathrm{SD}}+\tau_{\mathrm{RD}}\left|\psi_{j}^{t}\right|+\tau_{\mathrm{SR}}\left|\bar{\psi}_{j}^{t}\right|}\right\}$.

where $\beta_{0}=P_{0} / P$ and $\beta_{b}=P_{0} / P$ are the optimal source-relay power allocation factors. $T_{1}$ and $T_{2}$ are expressed in (66). ple phase $W(\mathrm{~m})$ protocol is shown in Fig. 4, considering that all the relays correctly decode and re-transmits information to the destination [44]. Let

$$
\begin{aligned}
T_{1}= & \left(\frac{4 M R_{c}}{b^{\prime}\left(\rho_{\mathrm{SR}}^{(b)}\right)^{2(t-1)} \sum_{l=1}^{|\mathrm{Y}|} \lambda_{\mathrm{ln}}^{2} \mathbf{c}_{2}\left(\sigma_{s, r}^{(b)}\right)^{2}}\right)^{\tau_{\mathrm{SR}}}\left\{\frac{a^{\prime}}{\pi} \int_{0}^{\frac{\pi}{2}}\left(\sin ^{2} \Phi\right)^{\tau_{\mathrm{SR}}} \mathrm{d} \Phi-\frac{c^{\prime}}{\pi} \int_{0}^{\frac{\pi}{4}}\left(\sin ^{2} \Phi\right)^{\tau_{\mathrm{SR}}} \mathrm{d} \Phi\right\}, \\
T_{2}= & \frac{a^{\prime}\left(4 M R_{c}\right)^{\tau_{\mathrm{SD}}+\tau_{\mathrm{RD}}}}{\pi\left(b^{\prime} \sum_{i=1}^{|\mathrm{Y}|} \lambda_{\mathrm{in}}^{2}\right)^{\tau_{\mathrm{SD}}+\tau_{\mathrm{RD}}}\left(\left(\rho_{\mathrm{SD}}\right)^{2(t-1)}\right)^{\tau_{\mathrm{SD}}}} \prod_{b \in \psi_{j}^{t}}\left\{\frac{1}{\left(\left(\rho_{\mathrm{RD}}^{(b)}\right)^{2(t-1)} \mathbf{c}_{1}\left(\sigma_{r, d}^{(b)}\right)^{2}\right)} \int_{0}^{\frac{\pi}{2}}\left(\sin ^{2} \Phi\right)^{\tau_{\mathrm{SD}+\tau_{\mathrm{RD}}}} \mathrm{d} \Phi\right\} \\
- & \frac{c^{\prime}\left(4 M R_{c}\right)^{\tau_{\mathrm{S} D}+\tau_{\mathrm{RD}}}}{\pi\left(b^{\prime} \sum_{i=1}^{|\mathrm{Y}|} \lambda_{\mathrm{in}}^{2}\right)^{\tau_{\mathrm{SD}+\tau_{\mathrm{RD}}}}\left(\left(\rho_{\mathrm{SD}}\right)^{2(t-1)}\right)^{\tau_{\mathrm{SD}}}} \prod_{b \in \psi_{j}^{t}}\left\{\frac{1}{\left(\left(\rho_{\mathrm{RD}}^{(b)}\right)^{2(t-1)} \mathbf{c}_{1}\left(\sigma_{r, d}^{(b)}\right)^{2}\right)} \int_{0}^{\frac{\pi}{4}}\left(\sin ^{2} \Phi\right)^{\tau_{\mathrm{SD}}+\tau_{\mathrm{RD}}} \mathrm{d} \Phi\right\} .
\end{aligned}
$$

where $P$ is the total available power budget and $\left|\bar{\psi}_{j}^{t}\right|$ represents the cardinality of the incorrectly decoding set. Considering for the quasi-static channel, we can assume that the state of the cooperation system will not change for every one of orthogonal STBC code word $B_{s}(t)$. For quasi-static channel conditions, we can write $\psi_{j}^{t}=\psi_{j}$ and $\bar{\psi}_{j}^{t}=\bar{\psi}_{j} \forall t$ in (65). The DO is the exponent of the $N_{0} / P$; one can readily see from (65) that the DO is expressed as

$\mathrm{DO}=\tau_{\mathrm{SD}}+\tau_{\mathrm{RD}}\left|\psi_{j}\right|+\tau_{\mathrm{SR}}\left|\bar{\psi}_{j}\right|$.

\section{Per-block average PEP investigation of $\mathrm{MH}$ multiple relay S-DF relaying network}

In Sect. 3, the per-block average PEP performance of $\mathrm{DH}$ relaying network is investigated over time-selective Nakagami- $m$ fading channel conditions. In this section, we investigate the $\mathrm{MH}$ relaying network presented as $W(\mathrm{~m})$; $(1 \leq m \leq L-1)$ with $L+1$ phases. $W(L-1)$ is most complex, $\psi_{j}^{t}(b)=\left\{\tilde{b} \mid \xi_{j}^{t}(\tilde{b}) \neq 0 ; \max \{1, b-\mathbf{m}\} \leq \tilde{b} \leq b\right\}$ represent the set corresponding to $b$ th relay, which includes all previous relays that successfully decode the MIMO-OSTBC code word [30]. Let $\sigma_{\in b \tilde{b}^{\prime}}^{2}\left(\sigma^{\tilde{b}, b}\right)^{2}, \rho^{b \tilde{b}}$ and $\sigma_{e b \tilde{b}}^{2}$ represent the channel error variance, channel gain, channel correlation coefficient, and channel error variance arise due to node mobility between the relay $b$ and $\tilde{b}$, respectively. In this relaying scheme, $b$ th relay combines the signals received from the past $\min \{\mathrm{m}, b-1\}$ relays along with that obtained from the source node and selectively forwards the OSTBC code words in $b+1$ phase. This is because the number of signals received at each relay node depends on the number of relays that have been correctly decoded from the previous $m$ relays. Similar to the $\mathrm{DH}$ relaying protocol, the average PEP of the $W(\mathrm{~m})$ protocol for the confusing event when $B_{s}(t)$ was confused with $B_{i}(t)$ is expressed as 
$P_{b}\left(B_{s}(t) \rightarrow B_{i}(t)\right)=\sum_{j=0}^{2^{L}-1} E_{\tilde{\gamma}}\left\{P_{b}\left(B_{s}(t) \rightarrow B_{i}(t) \mid \xi_{j}^{t}, \tilde{\gamma}\right)\right\} \times \prod_{b=1}^{L} E_{\tilde{\gamma}}\left\{P_{b}\left(\xi_{j}^{t}(b) \mid \underline{\xi}, \tilde{\gamma}\right)\right\}$

where $E_{\tilde{\gamma}}\left\{P_{b}\left(B_{s}(t) \rightarrow B_{i}(t) \mid \xi_{j}^{t}, \tilde{\gamma}\right)\right\}$, the average PEP for the error event $B_{s}(t) \rightarrow B_{i}(t)$, is expressed in (30) and is same for both $\mathrm{MH}$ and $\mathrm{DH}$ relaying protocols $[20,29]$. The average probability that the $b$ th relay being in a state $\xi_{j}^{t}(b)$ corresponding to the state of the previous $m$ relays is defined as $\underline{\xi}=\left[\xi_{j}^{t}(b-1), \xi_{j}^{t}(b-2), \ldots, \xi_{j}^{t}(b-\mathbf{m})\right]$ and CSI $\tilde{\gamma}=\left\{\gamma_{\mathrm{SD}}(t), \gamma_{\mathrm{SR}}^{(b)}(t), \gamma_{\mathrm{RD}}^{(b)}(t), \gamma^{b, \tilde{b}}(t), 1 \leq b \leq L, \max (1, b-\mathbf{m}) \leq \tilde{b} \leq b\right\}$ is expressed as $E_{\tilde{\gamma}}\left\{P_{b}\left\{\xi_{j}^{t}(b) \mid \underline{\xi}, \tilde{\gamma}\right\}\right\}[18]$, where $\gamma^{b, \tilde{b}}(t)$ represents the instantaneous SNR between $b$ th and $b$ th relay fading link expressed as $\gamma^{b, \tilde{b}}(t)=\mathbf{c}_{3} \bar{\gamma}^{b, \tilde{b}}\left\|\hat{A}^{b, \tilde{b}}(1)\right\|^{2}$, where $\mathbf{c}_{3}$ denotes the distance-dependent PTFs for $b$ th-to$b$ th fading links and $\bar{\gamma}^{b, \tilde{b}}$ is expressed below

$$
\begin{aligned}
\bar{\gamma}^{b, \tilde{b}}= & I^{\prime}\left(\rho^{b \tilde{b}}\right)^{2(t-1)}\left\{1+\mathrm{I}^{\prime}\left(\rho^{b \tilde{b}}\right)^{2(t-1)} \kappa \sigma_{\in b \tilde{b}}^{2}\right. \\
& \left.+\mathrm{I}^{\prime}\left(1-\left(\rho^{b \tilde{b}}\right)^{2(t-1)}\right) \kappa \sigma_{e b \tilde{b}}^{2}\right\}^{-1} .
\end{aligned}
$$

$\gamma^{b, \tilde{b}}(t)$ is Erlang distributed RVs with the degree of freedom $2 M^{2} m_{R R}$, having CDF and PDF can be expressed as
Fig. 5 Per-block average PEP performance of $\mathrm{MH}$ cooperative communication system for various values of fading severity parameters with $L=3, M_{b}=100,\left(\sigma_{s, d}\right)^{2}=$ $\left(\sigma_{s, r}^{b}\right)^{2}=40,\left(\sigma_{r, d}^{b}\right)^{2}=5$, $\rho_{\mathrm{SD}}=\rho_{\mathrm{SR}}^{(b)}=\rho_{\mathrm{RD}}^{(b)}=0.9561$, $\sigma_{e_{i}}^{2}=0.1, \sigma_{\epsilon_{i}}^{2}=0.01$

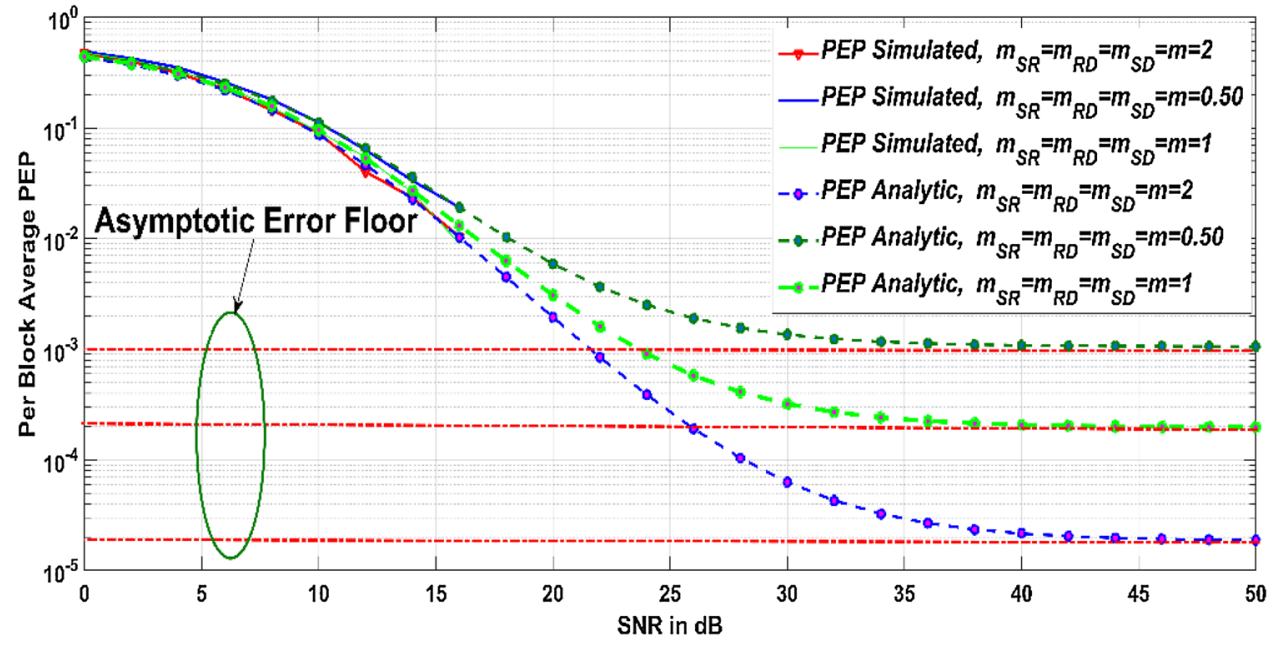

Fig. 6 Per-block average PEP performance of $\mathrm{MH}$ cooperative communication system for various values of RD channel gain with $L=3, M_{b}=100,\left(\sigma_{s, d}\right)^{2}=\left(\sigma_{s, r}^{b}\right)^{2}=10$,

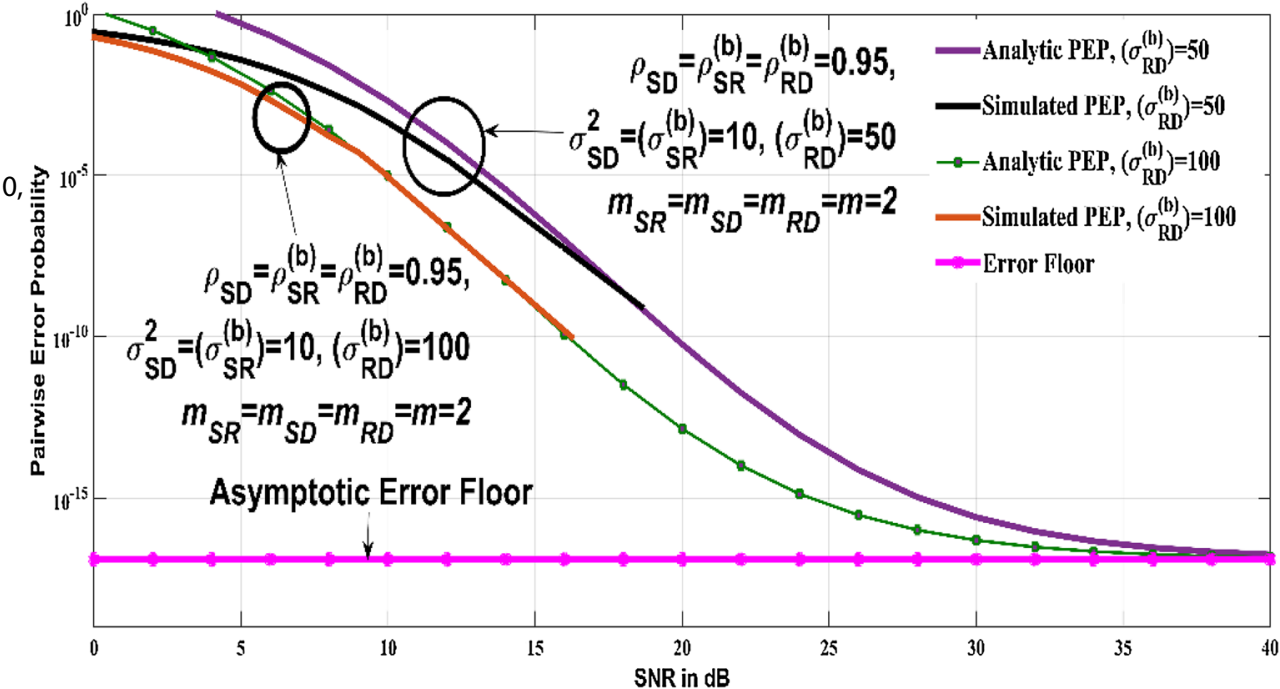




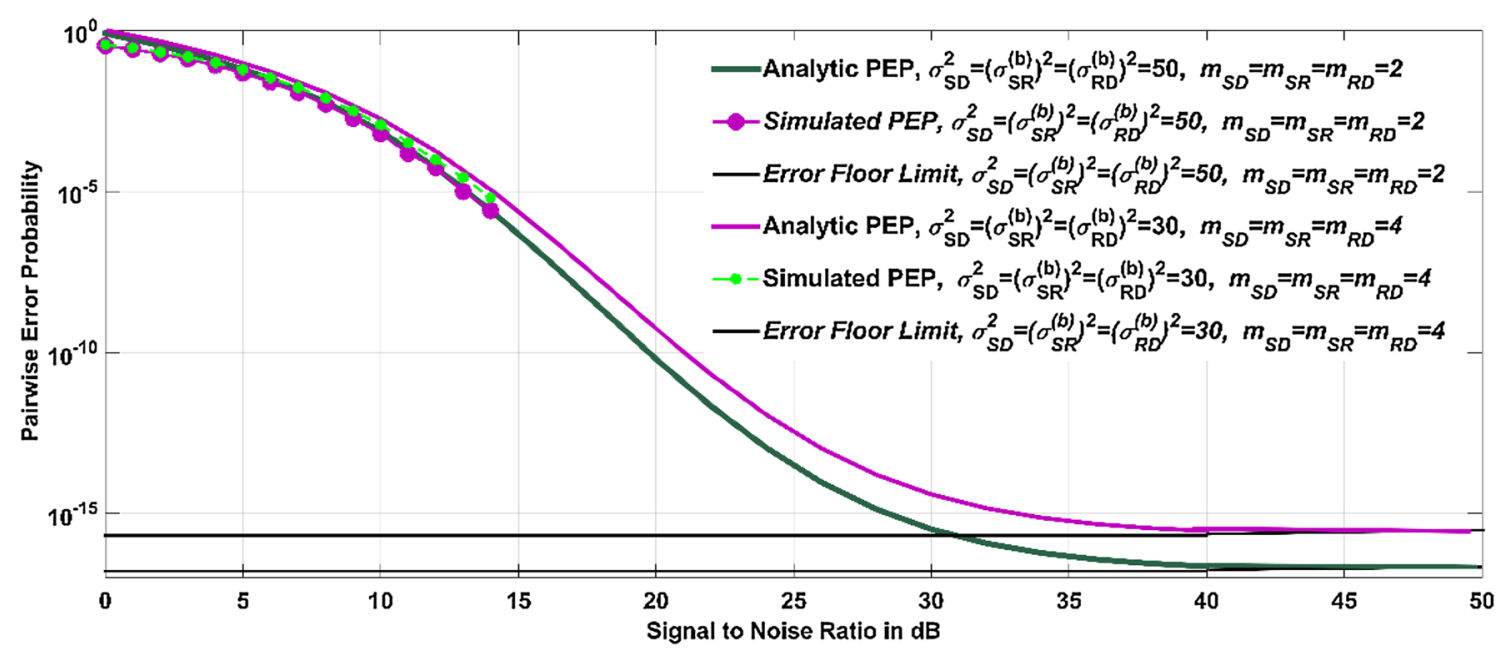

Fig. 7 Per-block average PEP performance of $\mathrm{MH}$ cooperative communication system for comparison between the RD channel gain and fading severity parameter with $L=3, M_{b}=50, \rho_{s, d}=\rho_{s, r}^{b}=\rho_{r, d}^{b}=0.9600, \sigma_{e_{i}}^{2}=0.3, \sigma_{\epsilon_{i}}^{2}=0.01$

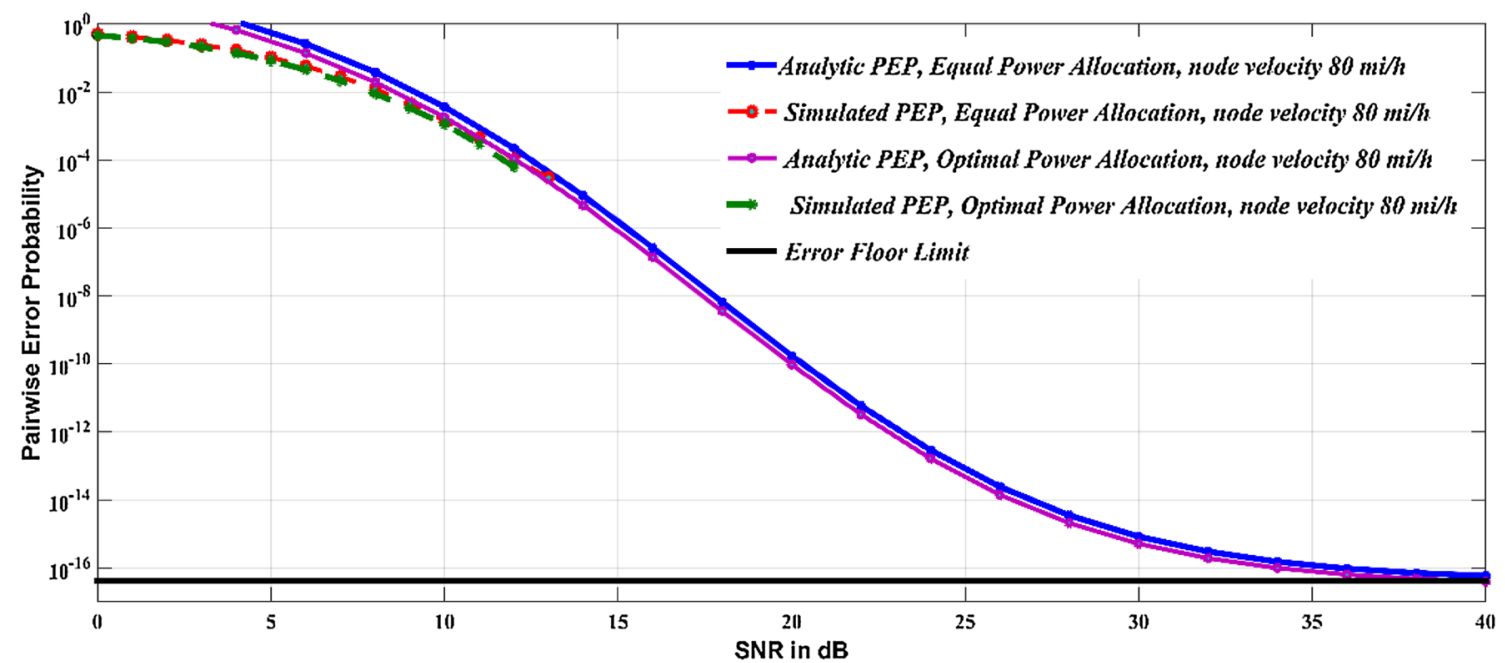

Fig. 8 Per-block average PEP performance of $\mathrm{MH}$ cooperative communication system for equal and optimal power allocation with $L=3, M_{b}=100,\left(\sigma_{s, d}\right)^{2}=\left(\sigma_{s, r}^{b}\right)^{2}=10,\left(\sigma_{r, d}^{b}\right)^{2}=100, \sigma_{e_{i}}^{2}=0.2, \sigma_{\epsilon_{i}}^{2}=0.02$ and optimal power factors are $\beta_{0}=0.7812, \beta_{1}=0.1094, \beta_{2}=0.1094$

$F_{\gamma^{b, \tilde{b}}(t)}(x)=\frac{\gamma\left(\tau_{\mathrm{RR}}, \frac{m_{\mathrm{RR}} x}{\mathbf{c}_{3}(\tilde{\sigma} b, \tilde{b})^{2}}\right)}{\Gamma\left(\tau_{\mathrm{RR}}\right)}$,

$f_{\gamma^{b, \tilde{b}}(t)}(x)=\frac{\left(\frac{1}{\mathbf{c}_{3}\left(\tilde{\sigma}^{b, \tilde{b}}\right)^{2}}\right)^{\tau_{\mathrm{RR}}}(x)^{\tau_{\mathrm{RR}}-1} \exp \left(-\frac{x}{\mathbf{c}_{3}\left(\tilde{\sigma}^{b, \tilde{b}}\right)^{2}}\right)}{\Gamma\left(\tau_{\mathrm{RR}}\right)}$, where $\quad \tau_{\mathrm{RR}}=m_{\mathrm{RR}} M^{2} \quad$ a n d $\quad\left(\tilde{\sigma}^{b, \tilde{b}}\right)^{2}=\left(\rho^{b \tilde{b}}\right)^{2(t-1)}$ $\left(\sigma^{b, \tilde{b}}\right)^{2}+\left(1-\left(\rho^{b \tilde{b}}\right)^{2(t-1)}\right) \sigma_{\in b \tilde{b}}^{2}$.The $b$ th relay may be in state $\xi_{j}^{t}(b)=1$, or it may be in state $\xi_{j}^{t}(b)=0$; the expression for the average error probability of the relaying network being in state in (68) can be expressed similarly to the DH protocol as [30] 


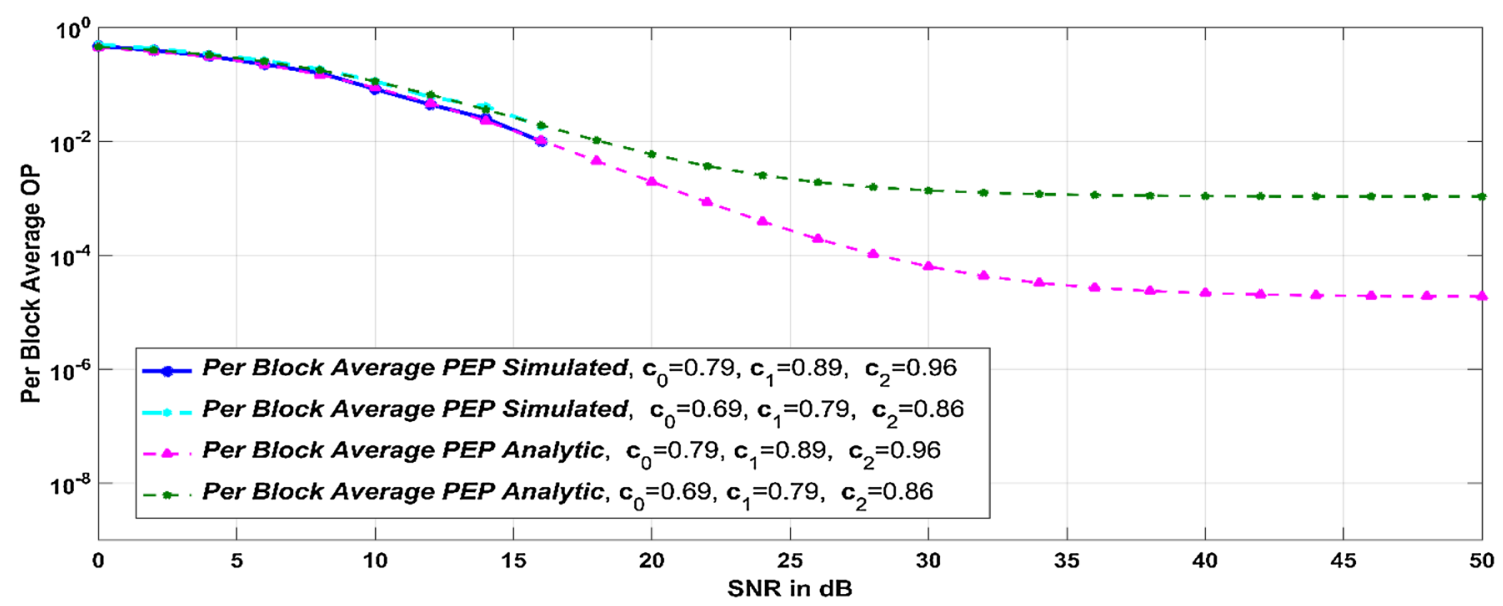

Fig. 9 Per-block average OP performance of DH cooperative communication system for various values distance-dependent power transfer factors with $L=3, M_{b}=100,\left(\sigma_{s, d}\right)^{2}=\left(\sigma_{s, r}^{b}\right)^{2}=\left(\sigma_{r, d}^{b}\right)^{2}=10, \sigma_{e_{i}}^{2}=0.1, \sigma_{\epsilon_{i}}^{2}=0.01$

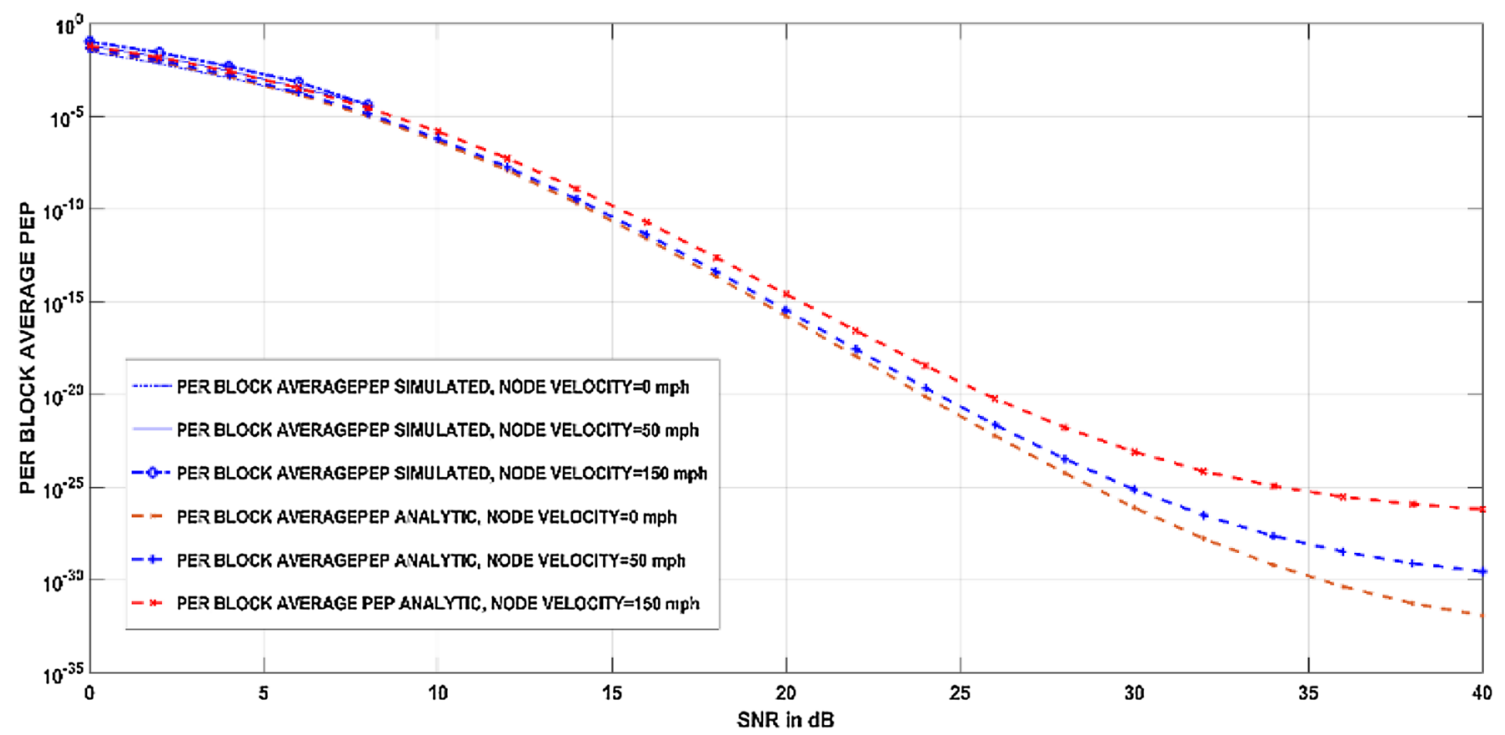

Fig. 10 Per-block average PEP performance of $\mathrm{DH}$ cooperative communication system for various nodes velocity with $L=3, M_{b}=10,\left(\sigma_{s, d}\right)^{2}=\left(\sigma_{s, r}^{b}\right)^{2}=20,\left(\sigma_{r, d}^{b}\right)^{2}=2, \sigma_{e_{i}}^{2}=0.15, \sigma_{\epsilon_{i}}^{2}=0.02, m=1$

$$
\begin{aligned}
& E_{\tilde{\gamma}}\left\{P_{b}\left(\xi_{j}^{t} / \tilde{\gamma}\right)\right\}=\prod_{b=1}^{L} E_{\tilde{\gamma}}\left\{P_{b}\left(\xi_{j}^{t}(b) / \underline{\xi}, \tilde{\gamma}\right)\right\} \\
& \quad=\prod_{b \in \tilde{\psi}_{j}^{t}}^{L}\left\{\sum_{l=1}^{|\gamma|} E_{\tilde{\gamma}}\left\{P_{e}^{S \rightarrow R_{b}}\left(B_{S}(t) \rightarrow B_{l}(t)\right)\right\}\right\} \times \prod_{b \in \psi_{j}^{t}}^{L}\left\{1-\sum_{l=1}^{|\gamma|} E_{\tilde{\gamma}}\left\{P_{e}^{S \rightarrow R_{b}}\left(B_{S}(t) \rightarrow B_{l}(t)\right)\right\}\right\} .
\end{aligned}
$$

where the expected PEP for the error event $B_{\mathrm{S}}(t) \rightarrow B_{l}(t)$ at $b$ th relay is denoted as $E_{\tilde{\gamma}}\left\{P_{e}^{S \rightarrow R_{b}}\left(B_{S}(t) \rightarrow B_{l}(t)\right)\right\}$ and can be expressed below

$$
E_{\tilde{\gamma}}\left\{P_{e}^{S \rightarrow R_{b}}\left(B_{S}(t) \rightarrow B_{l}(t)\right)\right\}=\left\{\left(\Xi_{1}-\Xi_{2}\right) \times \prod_{\tilde{b}=\max (1, b-m)}^{b-1}\left(\zeta_{1}-\zeta_{2}\right)\right\} .
$$




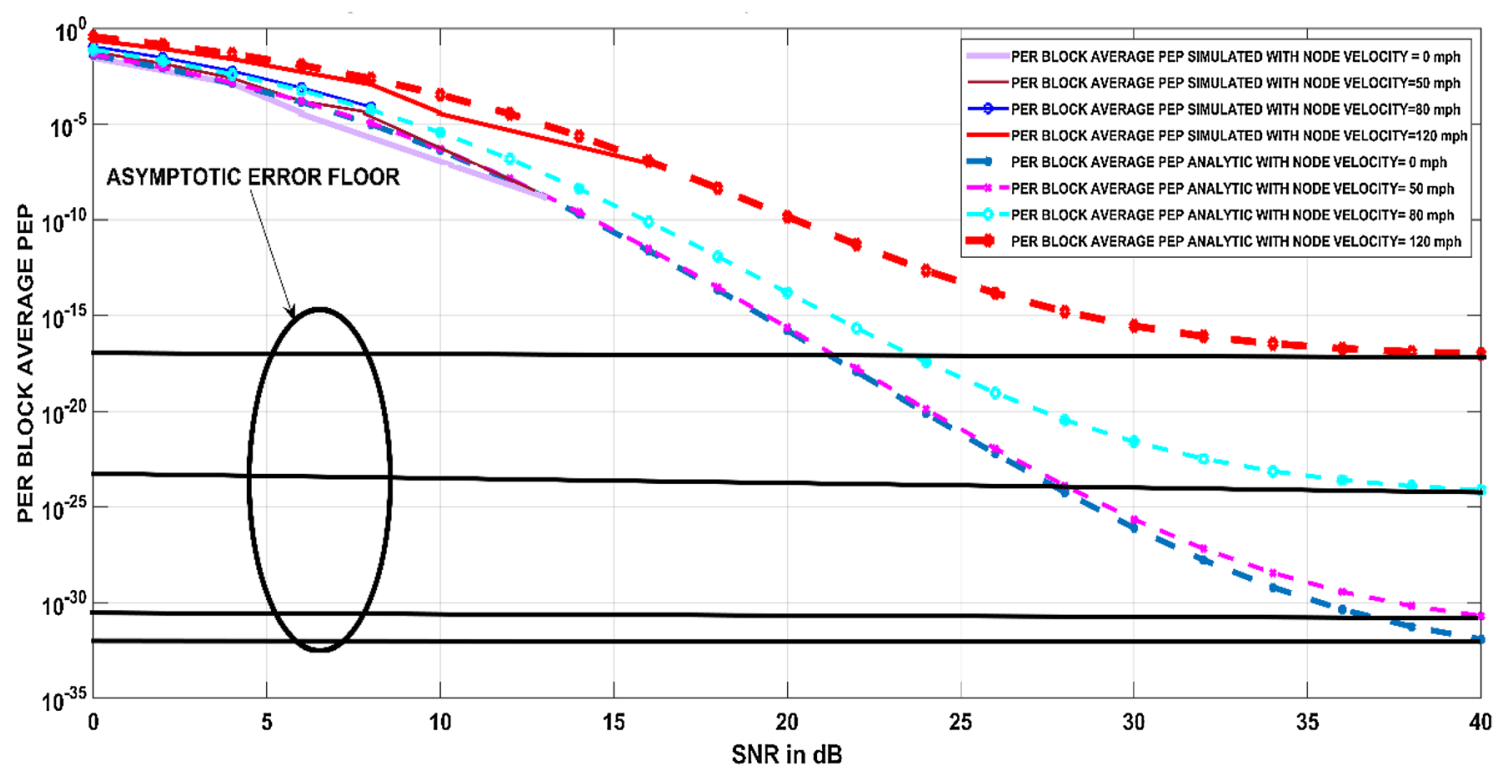

Fig. 11 Per-block average PEP performance of $\mathrm{MH}$ cooperative communication system for various nodes velocity with $L=3, M_{b}=15,\left(\sigma_{s, d}\right)^{2}=\left(\sigma_{s, r}^{b}\right)^{2}=\left(\sigma^{b, \tilde{b}}\right)^{2}=20,\left(\sigma_{r, d}^{b}\right)^{2}=2, \sigma_{e_{i}}^{2}=0.15, \sigma_{\epsilon_{i}}^{2}=0.02, m=1$

where $\zeta_{1}$ and $\zeta_{2}$ are given below

$\zeta_{1}=\frac{a^{\prime}}{2 \pi}\left(\frac{2 \tau_{\mathrm{RR}}}{2 \tau_{\mathrm{RR}}+\mathbf{c}_{3}\left(\sigma^{b, \tilde{b}}\right)^{2} b^{\prime} \lambda_{l}^{2} \bar{\gamma}^{b, \tilde{b}}}\right)^{\tau_{\mathrm{RR}}} \frac{\Gamma\left(\frac{1}{2}\right) \Gamma\left(\tau_{\mathrm{RR}}\right)}{\Gamma\left(\tau_{\mathrm{RR}}+1\right)} \times{ }_{2} F_{1}\left(\tau_{\mathrm{RR}}, \frac{1}{2} ; \tau_{\mathrm{RR}}+1 ; 1-\frac{1}{1+\frac{\mathbf{c}_{3}\left(\sigma^{b, \tilde{b}}\right)^{2} b^{\prime} \lambda^{2} \bar{\gamma}^{b}, \tilde{b}}{2 \tau_{\mathrm{RR}}}}\right)$,
$\zeta_{2}=\frac{c^{\prime}}{2 \sqrt{2 \pi}}\left(\frac{\mathbf{c}_{3}\left(\sigma^{b, \tilde{b}}\right)^{2} b^{\prime} \lambda_{l}^{2} \bar{\gamma}^{b, \tilde{b}}}{\tau_{\mathrm{RR}}}\right)^{\tau_{\mathrm{RR}}} \frac{\Gamma\left(\tau_{\mathrm{RR}}+\frac{1}{2}\right)}{\Gamma\left(\tau_{\mathrm{RR}}+\frac{3}{2}\right)} \times \mathbf{F}_{\mathrm{A}}^{(1)}\left(\tau_{\mathrm{RR}}+\frac{1}{2} ; \frac{1}{2}, \tau_{\mathrm{RR}} ; \tau_{\mathrm{RR}}+\frac{3}{2} ; \frac{1}{2} ;-\frac{\tau_{\mathrm{RR}}}{\left.\mathbf{c}_{3}\left(\sigma^{b, \tilde{b}}\right)^{2} b^{\prime} \lambda_{l}^{2} \bar{\gamma}^{b, \tilde{b}}\right) .}\right.$

Lastly, after substituting the formulation for $E_{\tilde{\gamma}}\left\{P_{b}\left(B_{s}(t) \rightarrow B_{i}(t) \mid \xi_{j}^{t}, \tilde{\gamma}\right)\right\}$ from the expression given in (30) along with the formulation for $E_{\tilde{\gamma}}\left\{P_{b}\left(\xi_{j}^{t}, \tilde{\gamma}\right)\right\}$ given in $\bar{P}_{e}^{F} \leq \frac{1}{M_{\mathrm{B}}} \sum_{t=1}^{M_{\mathrm{B}}} \sum_{s=1}^{|\mathrm{Y}|} P_{b}\left(B_{s}(t)\right) \sum_{i=1, s \neq i}^{|\mathrm{Y}|} P_{b}\left(B_{s}(t) \rightarrow B_{i}(t)\right)$.

(71) into (68), one can express the expected expression of PEP for error event $B_{s}(t) \rightarrow B_{i}(t)$ as

$$
\begin{aligned}
& P_{b}\left(B_{S}(t) \rightarrow B_{i}(t) / \underline{\xi}, \tilde{\gamma}\right) \\
& \quad=\sum_{j=0}^{2^{L}-1}\left\{\left\{\prod_{b \in \tilde{\psi}_{j}^{t}}^{L}\left\{\sum_{l=1}^{|x|} E_{\tilde{\gamma}}\left\{P_{e}^{S \rightarrow R_{b}}\left(B_{S}(t) \rightarrow B_{l}(t)\right)\right\}\right\} \times \prod_{b \in \psi_{j}^{t}}^{L}\left\{1-\sum_{l=1}^{|x|} E_{\tilde{\gamma}}\left\{P_{e}^{S \rightarrow R_{b}}\left(B_{S}(t) \rightarrow B_{l}(t)\right)\right\}\right\}\right\} \times\left\{\left(\mho_{1}-\mho_{2}\right) \prod_{b \in \psi_{j}^{t}}\left(\mathrm{O}_{1}-\mathrm{O}_{2}\right)\right\}\right) .
\end{aligned}
$$

The per-block average upper union bound expression can be expressed as 


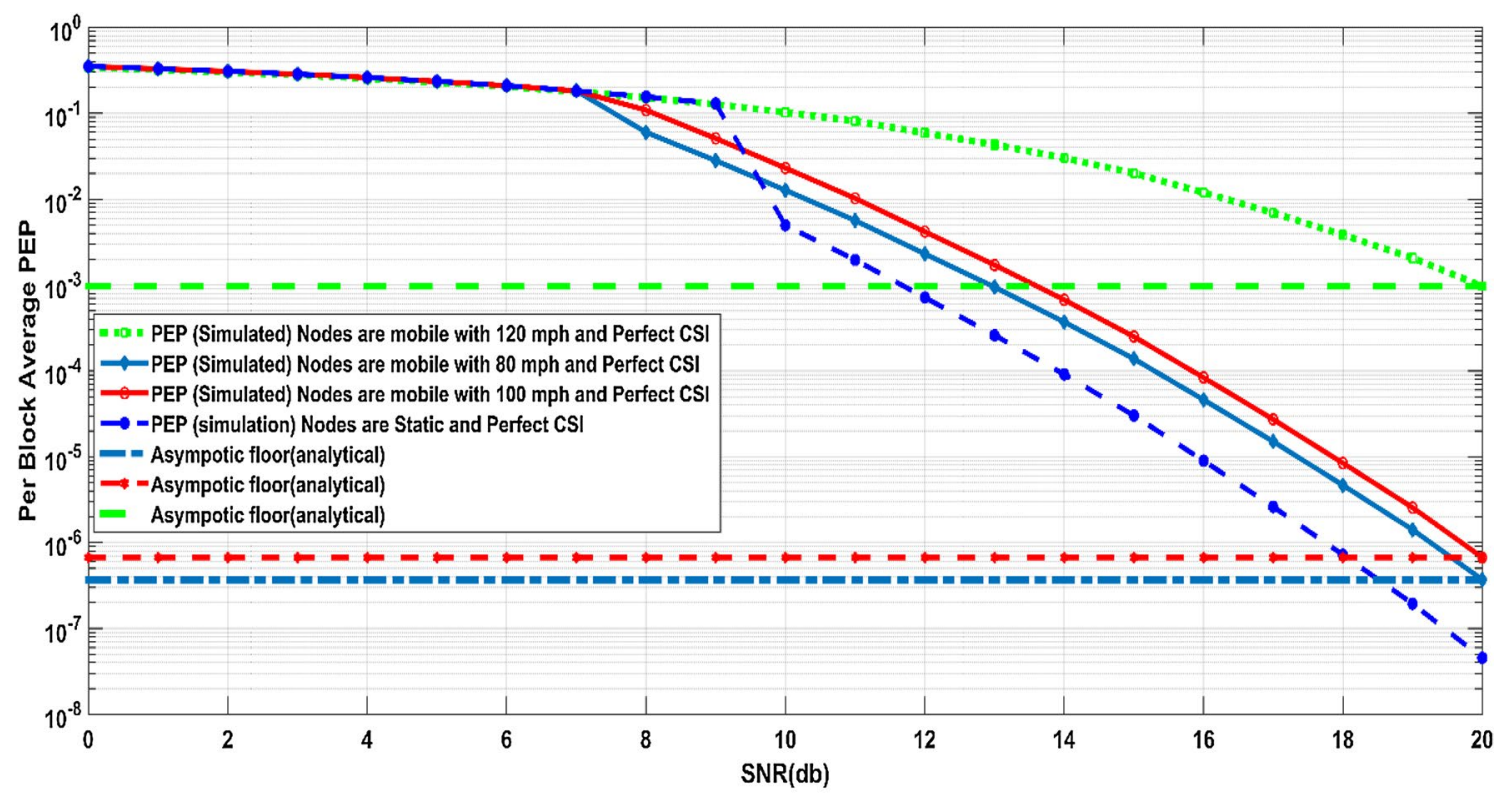

(a)

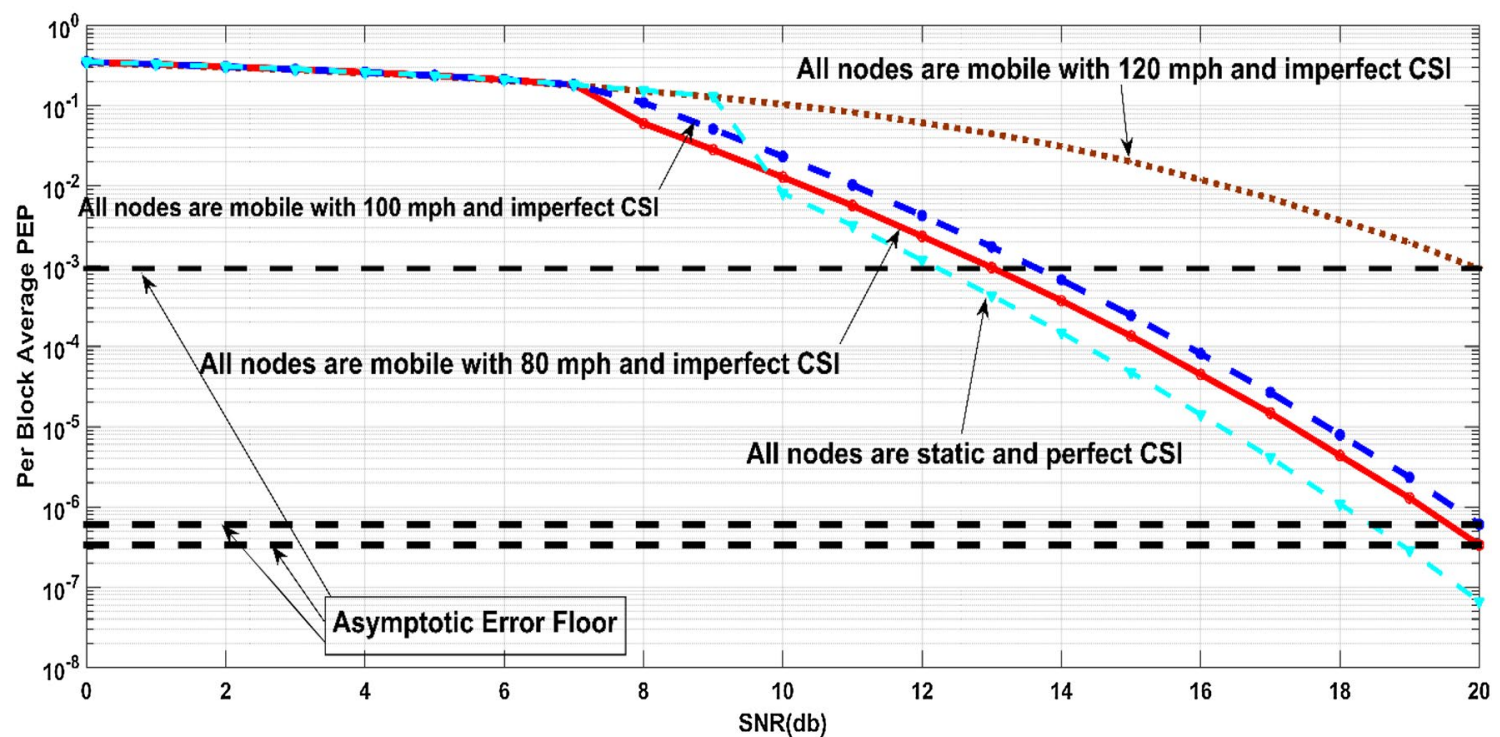

(b)

Fig. 12 Per-block average PEP performance of Alamouti STBC a $W(1)$ and b $W(2)$ relaying networks with $m=1, L=3, M_{b}=10,\left(\sigma_{s, d}\right)^{2}=\left(\sigma_{s, r}^{b}\right)^{2}=\left(\sigma^{b, \tilde{b}}\right)^{2}=20,\left(\sigma_{r, d}^{b}\right)^{2}=2, \sigma_{e_{i}}^{2}=0.15, \sigma_{\epsilon_{i}}^{2}=0.02$ 


\subsection{Asymptotic floor for per-frame average PEP}

Further, following the similar procedure adopted for finding the error floor limit for $\mathrm{DH}$ protocol given in Sect. 3, the per-block average asymptotic error floor for $\mathrm{MH} \mathrm{W(m)}$ protocol is given as sidering the dominating terms corresponding to $m, n=0,1$ in the identity ${ }_{2} F_{1}(a, b ; c ; z)=\sum_{n=0}^{K} \frac{(a)_{n}(b)_{n} z^{n}}{(c)_{n} n !}$, for the Gauss hypergeometric function and Appell hypergeometric function of two variables,

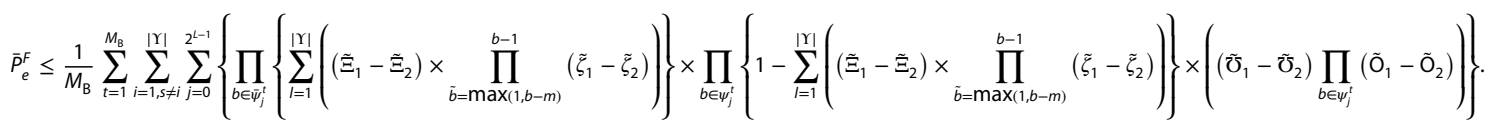

where $\left(\tilde{\mho}_{1}-\tilde{\mho}_{2}\right)$ and $\left(\tilde{O}_{1}-\tilde{O}_{2}\right)$ are expressed in (64) and

$\tilde{\zeta}_{1}-\tilde{\zeta}_{2}$ is expressed in (77).

$\tilde{\zeta}_{1}-\tilde{\zeta}_{2}$

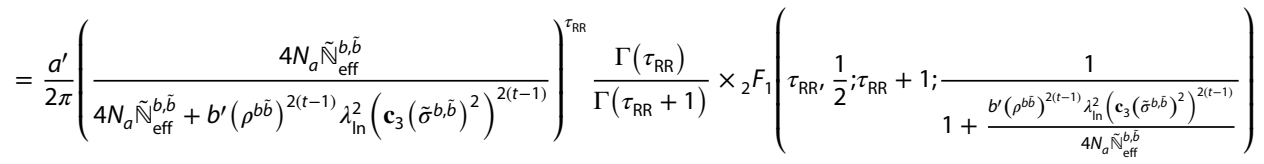

$$
\begin{aligned}
& -\frac{c^{\prime}}{\pi}\left(\frac{4 N_{a} \tilde{\mathbb{N}}_{\mathrm{eff}}^{b, \tilde{b}}}{4 N_{a} \tilde{\mathbb{N}}_{\mathrm{eff}}^{b, \tilde{b}}+b^{\prime}\left(\rho^{b \tilde{b}}\right)^{2(t-1)} \lambda_{\ln }^{2}\left(\mathbf{c}_{3}\left(\tilde{\sigma}^{b, \tilde{b}}\right)^{2}\right)^{2(t-1)}}\right)^{\tau_{\mathrm{RR}}} \Gamma \frac{\Gamma\left(\tau_{\mathrm{RR}}+\frac{1}{2}\right)}{\Gamma\left(\tau_{\mathrm{RR}}+\frac{3}{2}\right)} \times \mathbf{F}_{\mathrm{A}}^{(1)}\left(\tau_{\mathrm{RR}}+\frac{1}{2} ; \tau_{\mathrm{RR}}, \frac{1}{2} ; \tau_{\mathrm{RR}}+\frac{3}{2} ; \frac{2 b^{\prime}\left(\rho^{b \tilde{b}}\right)^{2(t-1)} \lambda_{\ln }^{2}\left(\mathbf{c}_{3}\left(\tilde{\sigma}^{b, \tilde{b}}\right)^{2}\right)^{2(t-1)}}{4 N_{a} \tilde{\mathbb{N}}_{\mathrm{eff}}^{b, \tilde{b}}} ; \frac{1}{2}\right),
\end{aligned}
$$

where $\quad \tilde{\mathbb{N}}_{\text {eff }}^{b, \tilde{b}}=\left(N_{0}+\left(\rho^{b \tilde{b}}\right)^{2(t-1)} \tilde{\sigma}_{\epsilon b \tilde{b}}^{2}+\left(1-\left(\rho^{b \tilde{b}}\right)^{2(t-1)}\right) \tilde{\sigma}_{e b \tilde{b}}^{2}\right)$, $\left(\tilde{\sigma}^{b, \tilde{b}}\right)^{2}=\left(\sigma^{b, \tilde{b}}\right)^{2}+\sigma_{\in b \tilde{b}^{\prime}}^{2}$ and $\tilde{\sigma}_{e b \tilde{b}}^{2}=N_{a} \sigma_{e b \tilde{b}}^{2}$. The error terms $\tilde{\sigma}_{\in b \tilde{b}}^{2}$ and $\tilde{\sigma}_{e b \tilde{b}}^{2}$ are equivalent to $N_{a} \sigma_{\in b \tilde{b}^{\prime}}^{2}$ and $N_{a} \sigma_{e b \tilde{b}^{\prime}}^{2}$ respectively. If the relay nodes are mobile and source nodes and destination nodes are immobile, then we get $\mathrm{DO}$ of $\tau_{\mathrm{RD}}$. The $\mathrm{DH}$ relaying network experiences asymptotic error floor due to time selectivity except for perfect CSI and static conditions.

\subsection{Analysis of DO and OPA}

In this subsection, DO is analyzed and a mathematical model is developed to obtain the optimal source-relay power allocation factors, which will further enhance the end-to-end error performance of the relaying network. Like $\mathrm{DH}$ relaying protocol, for static nodes and perfect $\mathrm{CSI}$ conditions, quasi-static fading is considered; hence, we can take $\psi_{j}^{t}=\psi_{j}$ and $\bar{\psi}_{j}^{t}=\bar{\psi}_{j} \forall t$. For perfect CSI and static nodes, we take $\sigma_{\in S D}^{2}=\sigma_{\in S R}^{2}=\sigma_{\in \mathrm{RD}}^{2}=\sigma_{\in b \tilde{b}}^{2}=0$ and $\sigma_{\mathrm{eSD}}^{2}=\sigma_{\mathrm{eSR}}^{2}=\sigma_{\mathrm{eRD}}^{2}=\sigma_{e b \tilde{b}}^{2}=0$, respectively. The state of the cooperation network $\xi_{j}^{t}$ is same for every code word $B_{\mathrm{S}}(t), t=1,2,3, \ldots, M_{b}$ in this scheme. Applying the abovementioned conditions in (74) and at high SNR approximating, $\prod_{b \in \psi_{j}^{t}}\left\{1-E_{\tilde{\gamma}}\left\{P_{e}^{S \rightarrow R_{b}}\left(B_{S}(t) \rightarrow B_{l}(t)\right)\right\}\right\} \approx 1$ and con- 
$\mathrm{F}_{\mathrm{A}}^{(1)}\left(\chi_{1}, l_{1}, l_{1}^{\prime}, \gamma^{\prime \prime} ; x^{\prime}, y^{\prime}\right)=\sum_{m, n=0}^{\infty} \frac{\left(\chi_{1}\right)_{m+n}\left(l_{1}\right)_{m}\left(l_{1}^{\prime}\right)_{n}}{\left(\gamma^{\prime \prime}\right)_{m+n} \Gamma(m+1) \Gamma(n+1)}\left(x^{\prime}\right)^{m}\left(y^{\prime}\right)^{n}$,

where $(x)_{n}$ is the Pochhammer symbols, one can simplify the expression in (74), as given in (78).

pasy

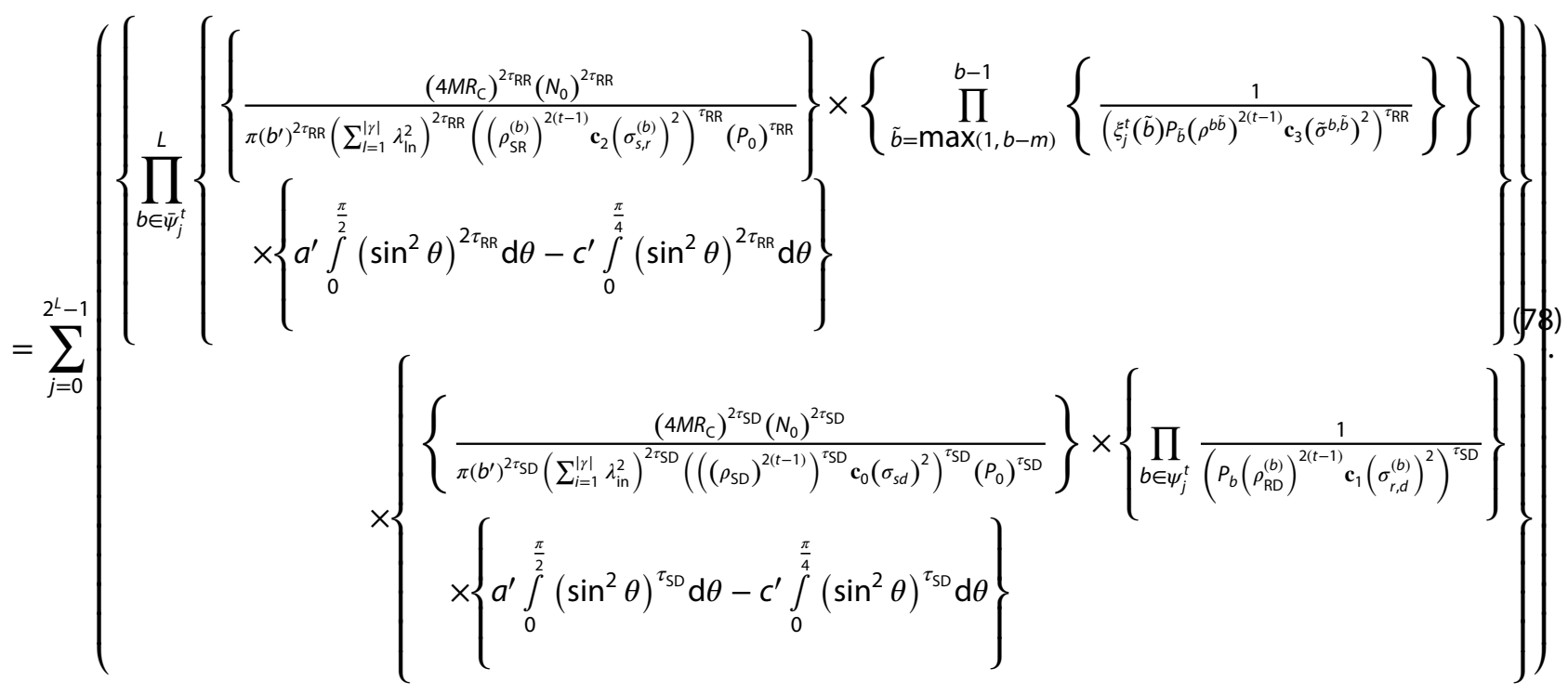

Further, (78) can be expressed in consolidated form as

$P^{\text {asy }} \leq \sum_{i=1}^{2^{L}-1} T_{c}\left(\frac{N_{0}}{P}\right)^{\mathrm{DO}_{j}}$,

where $T_{c}$ is expressed in (80). The exponent of $N_{0} / P$ in (79) basically represents the $\mathrm{DO}$, and it is expressed as $\mathrm{DO}_{j}$ definedasDO ${ }_{j}=\tau_{\mathrm{SD}}+\tau_{\mathrm{RD}}\left|\psi_{j}\right|+\tau_{\mathrm{SR}}\left|\bar{\psi}_{j}\right|+\tau_{\mathrm{RR}} \sum_{b \in \bar{\psi}_{j}}\left|\psi_{j}(b)\right|$. Lastly, the end-to-end error probability can be attained by doing summation of all the PEP terms of the confusing event $B_{s}(t) \rightarrow B_{i}(t)$ over all possible OSTBC code words, which is obtained as given in (80).
$\bar{P}^{\text {asy }} \leq \sum_{i=1}^{|\gamma|} \sum_{j=1}^{2^{L}-1} T_{c}\left(\frac{N_{0}}{P}\right)^{\mathrm{DO}_{j}}$.

The expression given in (80) can be as lower bounded (LB) as

$$
\tau_{\mathrm{SD}}+\tau_{\mathrm{RD}}\left|\psi_{j}\right|+\tau_{\mathrm{SR}}\left|\bar{\psi}_{j}\right| \quad \text { if } \quad \sum_{b \in \bar{\psi}_{j}}\left|\psi_{j}(b)\right| \quad \text { i. e. . }
$$
$\left|\psi_{j}(b)\right|=0 \forall b \in \bar{\psi}_{j}$, which is only possible for the network s t a t e s $\xi_{0}^{t}=[0,0, \ldots, 0], \xi_{1}^{t}=[0,0, \ldots, 0,1], \xi_{2}^{t}=$ $[0,0, \ldots 1,1], \ldots, \xi_{2^{L}-1}^{t}=[1,1,1, \ldots, 1,1,1]$. The optimal source-relay allocation power factors $\beta_{0}, \beta_{\tilde{b}} ; \tilde{b}=\max (1, b-\mathrm{m})$ for $\mathrm{MH} W(\mathrm{~m})$ cooperation protocol can be achieved as the solution of $\mathrm{CO}$ problem given as

$\min _{\beta_{0}, \beta_{1}, \beta_{2}, \ldots, \beta_{\tilde{b}}} \sum_{j=0}^{2^{L}-1}\left\{\left\{\prod_{b \in \bar{\psi}_{j}^{t}}^{L}\left\{\frac{K_{1}^{\prime}}{\left(\beta_{0}\right)^{M^{2}}} \prod_{\tilde{b}=\max (1, b-\mathrm{m})}^{b-1}\left\{\frac{1}{\left(\beta_{\tilde{b}}\right)^{M^{2}}}\right\}\right\}\right\} \times\left\{\frac{1}{\left(\beta_{0}\right)^{M M_{\mathrm{D}}}}\right\}\left\{\prod_{b \in \psi_{j}^{t}} \frac{1}{\left(\beta_{b}\right)^{M M_{\mathrm{D}}}}\right\}\right)$

s.t. $\beta_{0}+\beta_{1}+\cdots+\beta_{\tilde{b}} \leq 1$.

where $K_{1}^{\prime}$ is appropriately defined constant terms. The CO problem given in (81) is a GP, and it can be solved by using 


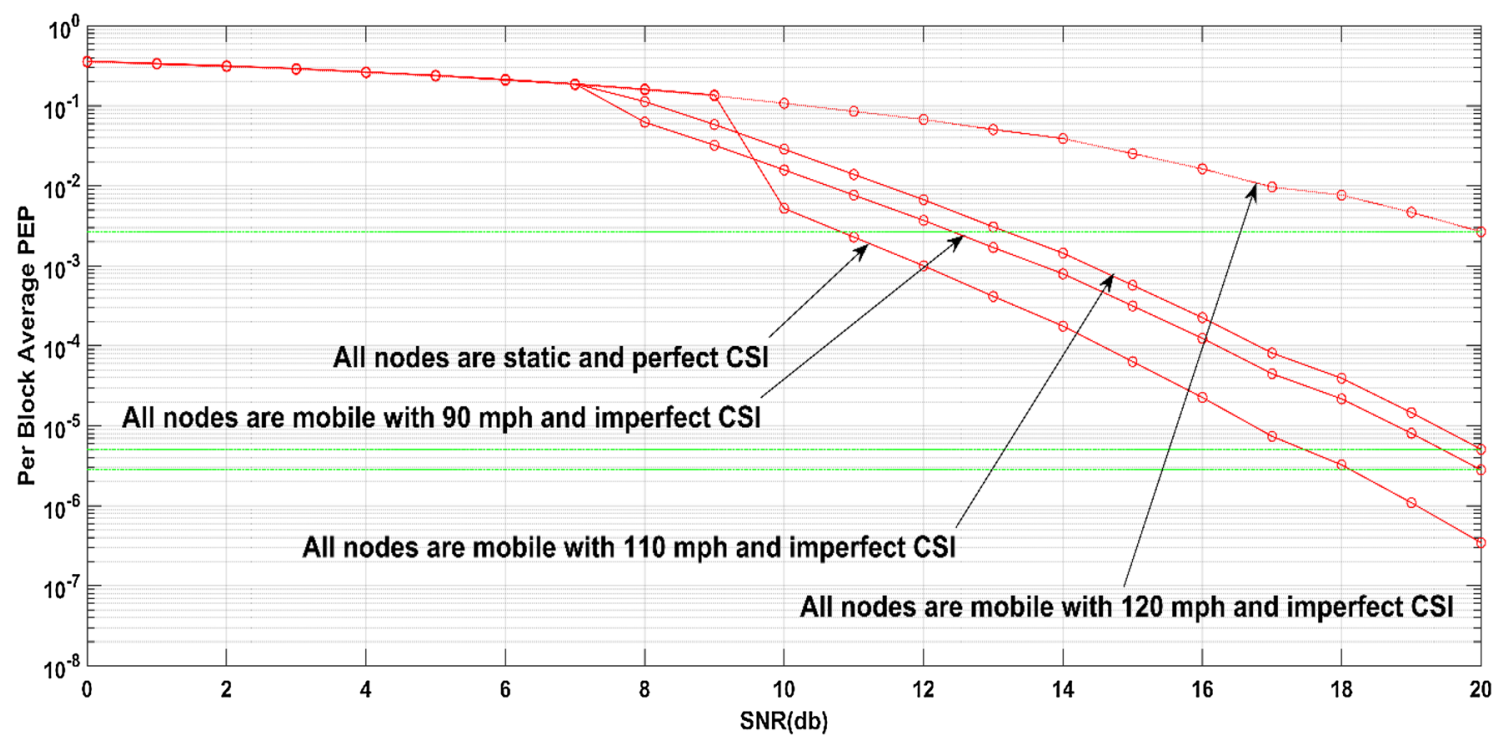

Fig. 13 Per-block average PEP performance DH dual-phase MIMO non-orthogonal STBC $(2 \times 2 \mathrm{GC}) W(1)$ relaying network with $L=3, m=1, M_{b}=15,\left(\sigma_{s, d}\right)^{2}=\left(\sigma_{s, r}^{b}\right)^{2}=\left(\sigma^{b, \tilde{b}}\right)^{2}=20,\left(\sigma_{r, d}^{b}\right)^{2}=2, \sigma_{e_{i}}^{2}=0.15, \sigma_{\epsilon_{i}}^{2}=0.02$

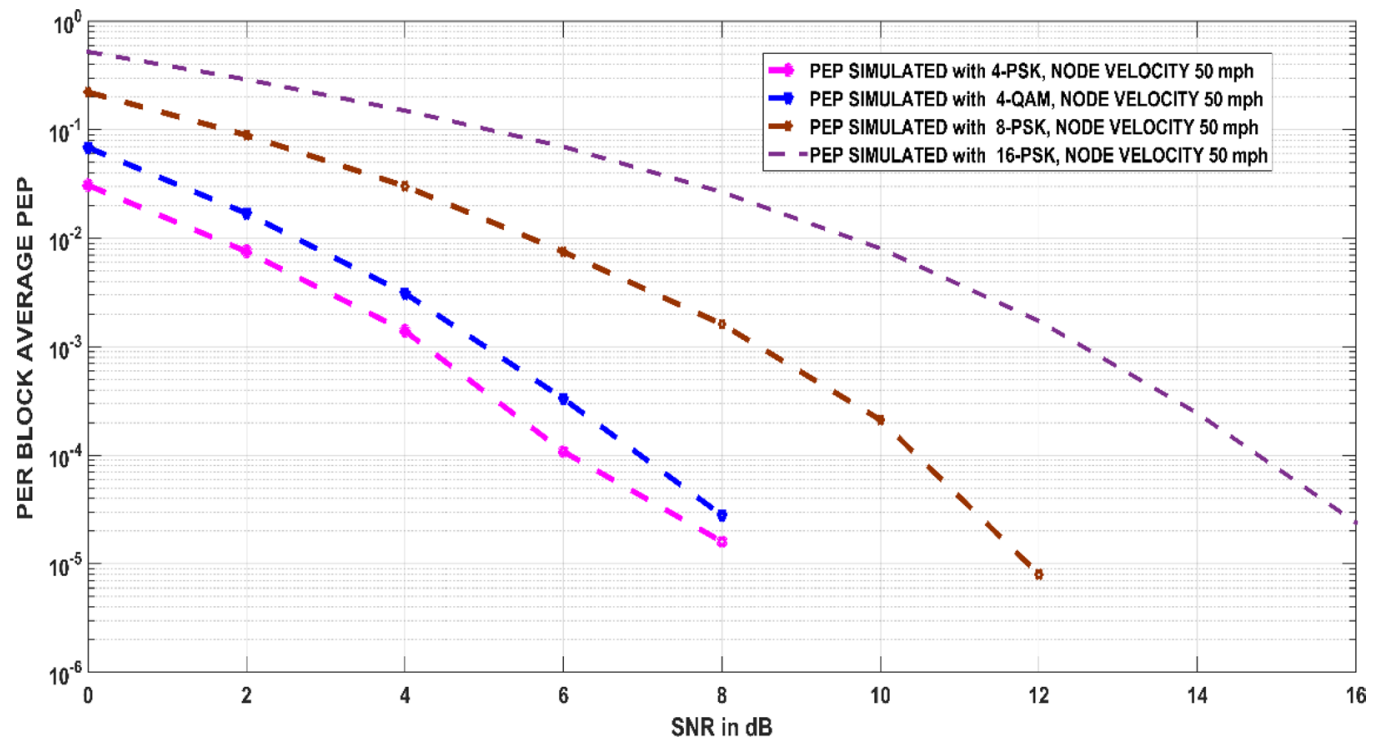

Fig. 14 Per-block average PEP performance of $\mathrm{MH}$ cooperative communication system for various modulation schemes with $L=3, m=1, M_{b}=20,\left(\sigma_{s, d}\right)^{2}=\left(\sigma_{s, r}^{b}\right)^{2}=\left(\sigma^{b, \tilde{b}}\right)^{2}=20,\left(\sigma_{r, d}^{b}\right)^{2}=2, \sigma_{e_{i}}^{2}=0.15, \sigma_{\epsilon_{i}}^{2}=0.02$ 


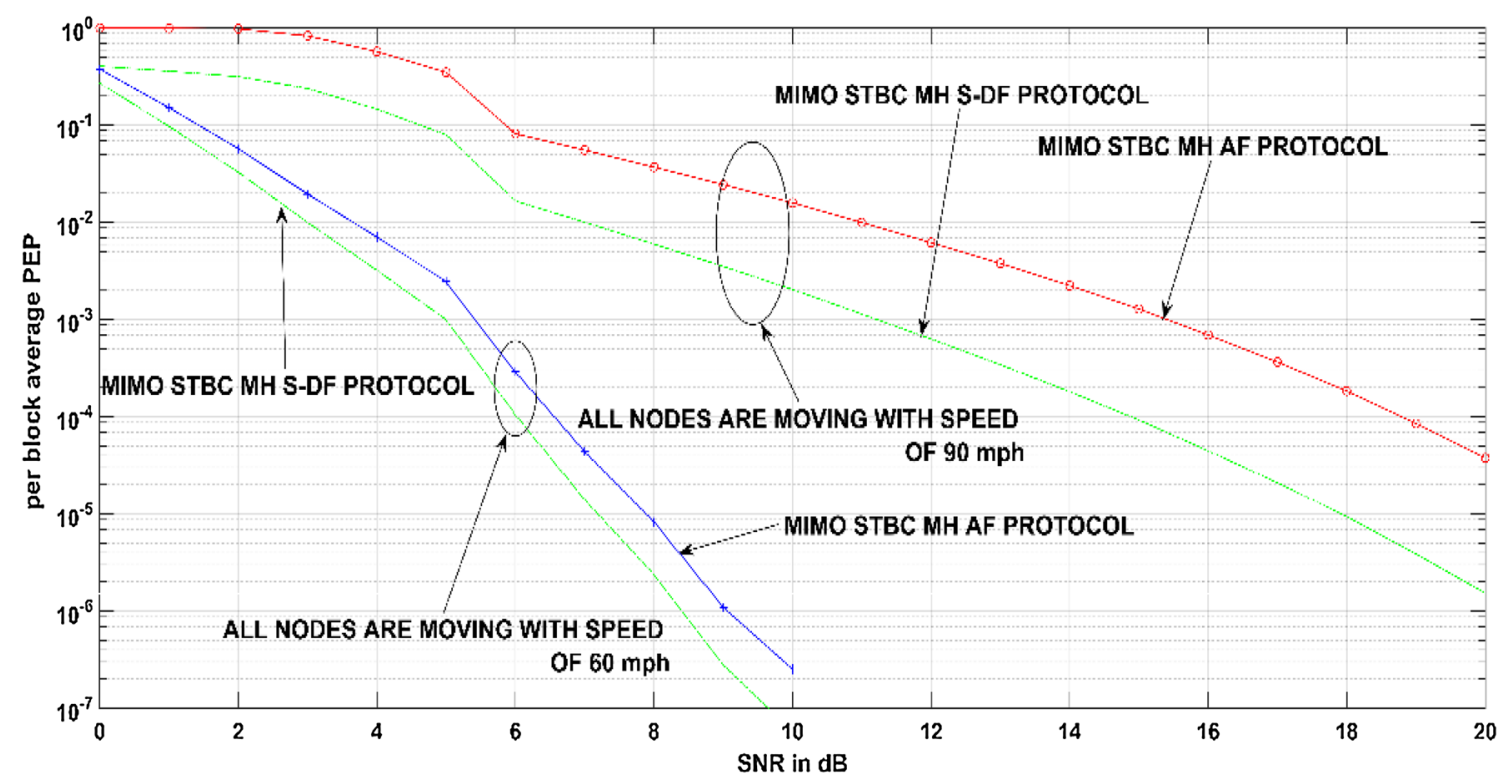

Fig. 15 Performance comparison between the S-DF and AF relaying protocols for 4-QAM with $L=3, m=1, M_{b}=15,\left(\sigma_{s, d}\right)^{2}=\left(\sigma_{s, r}^{b}\right)^{2}=\left(\sigma^{b, \tilde{b}}\right)^{2}=15,\left(\sigma_{r, d}^{b}\right)^{2}=2, \sigma_{e_{i}}^{2}=0.15, \sigma_{\epsilon_{i}}^{2}=0.02$

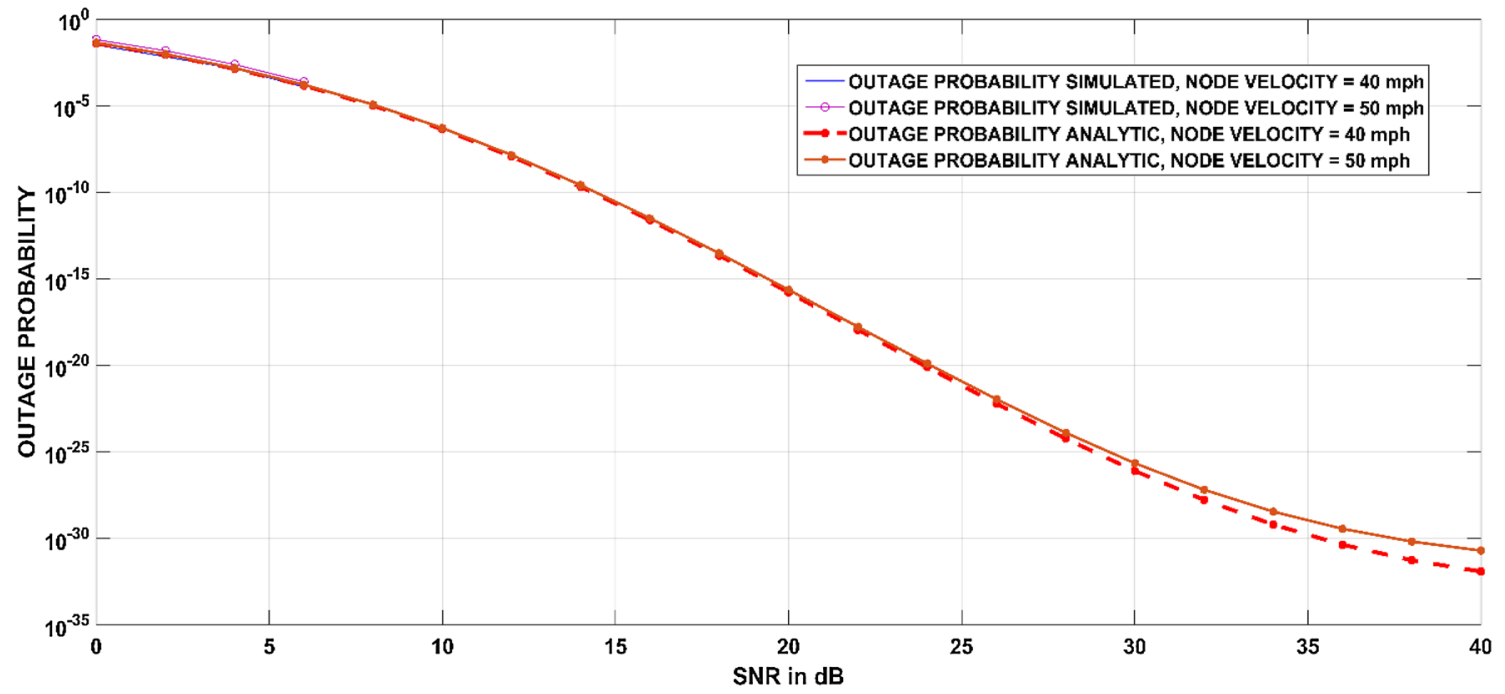

Fig. 16 Per-block average outage probability performance of DH cooperative communication system with $L=3, m=1, M_{b}=10,\left(\sigma_{s, d}\right)^{2}=\left(\sigma_{s, r}^{b}\right)^{2}=20,\left(\sigma_{r, d}^{b}\right)^{2}=2, \sigma_{e_{i}}^{2}=0.15, \sigma_{\epsilon_{i}}^{2}=0.02$ 
the CVX solver software for multiple relay case, and for the single relay case we can use the Karush-Kuhn-Tucker (KKT) [55]-based Lagrangian framework for finding the solution of the CO problem already discussed in the previous section. of $m$ improves the per-block average PEP performance. Figure 6 shows the per-block average PEP performance in which $v=96 m_{i} / h$ and 4-QAM modulation symbols are used. Plots show that increasing the value of RD fading link gain improves the per-block average PEP performance.

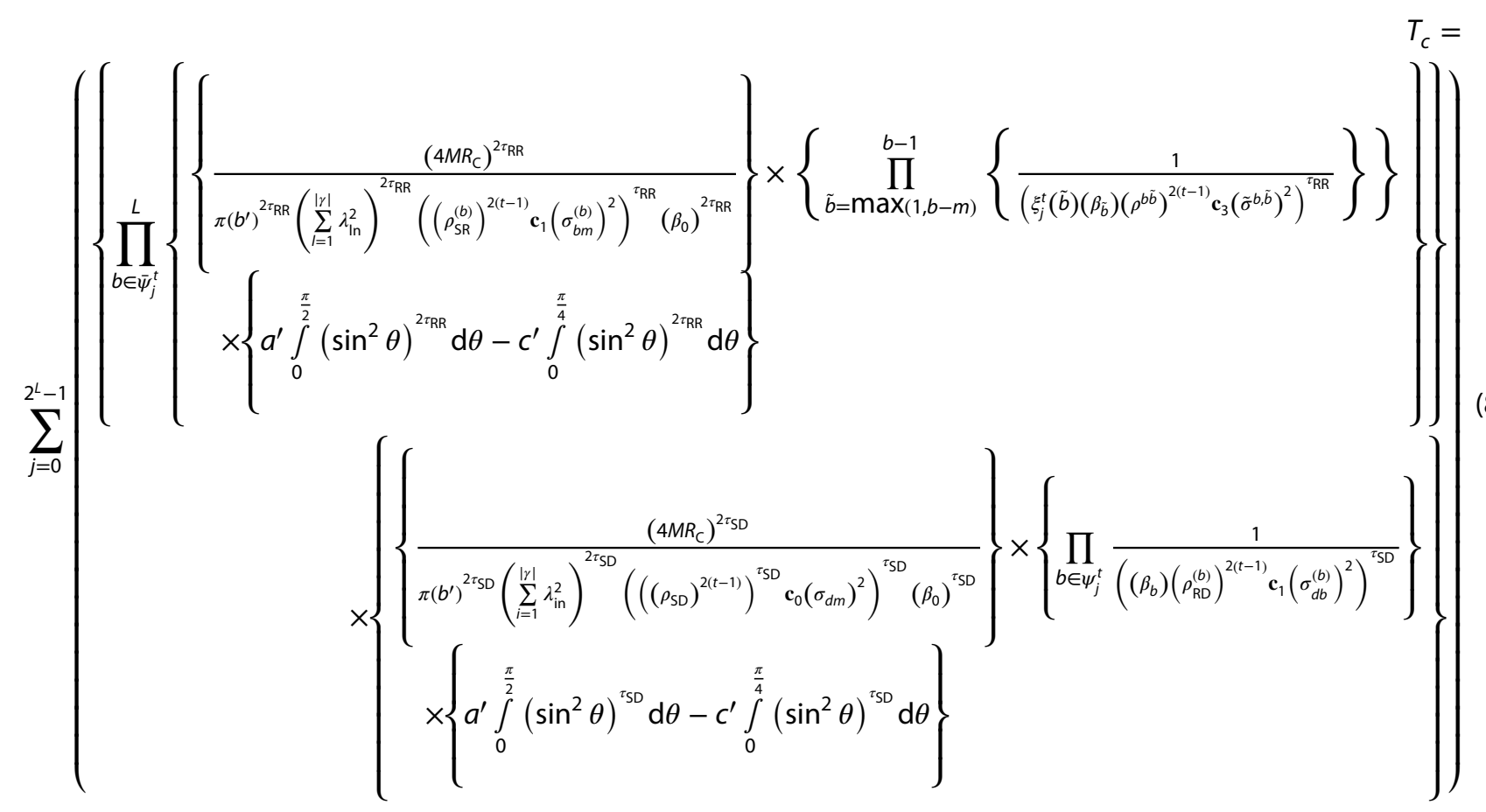

\section{Simulation results and discussion}

MC simulations are conducted for confirming the accuracy of the theoretical results for the considered S-DF cooperative communication protocol. We consider i.n.i.d. time-selective Nakagami- $m$ fading channel distributions with channel estimation error. Relay nodes are employed by the S-DF protocol and will only forward correctly decoded code word. In the simulation, we consider the Alamouti code word, distance-dependent PTFs are set as $\mathbf{c}_{0}=0.79, \mathbf{c}_{1}=0.89, \mathbf{c}_{2}=0.96, \mathbf{c}_{3}=0.69$ and outage threshold as $\gamma_{0}=6 \mathrm{~dB}$. The source, relays, and destination will get equal power unless stated otherwise. We employ M-ary modulation symbols for checking the accuracy of the per-block average PEP and per-block average OP investigation in the following experiments. The simulation parameters are given as $M=M_{\mathrm{D}}=2$ (for Alamouti Code), $R_{\mathrm{S}}=10 \mathrm{kbps}, f_{c}=6 \mathrm{GHz}$. Monte Carlo simulation outcomes displayed in Figs. 5, 6, 7 and 8 give important results for relay nodes, which are as follows. The per-block average PEP plots are in exact match with the simulation plots in the high SNR regimes. In Fig. 5, curves show the per-block average PEP performance in which $v=90 \mathrm{~m}_{i} / \mathrm{h}$ and $c=3 \times 10^{8} \mathrm{~m} / \mathrm{s}$ and 4-PSK modulation symbols are used. Plots show that increasing the value
The reason for performance improvement is that when the link gain of RD is very high, almost all the available power is allotted to the source node only and there is a better chance of getting the error-free code word at the relay nodes. Plots show that due to time-selective nature of the fading links, the per-block average PEP performance is degraded and achieves the asymptotic error floor limit when $P_{\mathrm{S}} / N_{0}$ and $P_{b} / N_{0} \rightarrow \infty$. In comparison, Fig. 7 shows the end-to-end system performance in which $m\{2,4\}$, $\left(\sigma_{r, d}^{b}\right)^{2}=\{30,50\}, v=96 m_{i} / h$, and 4-PSK are used. Perblock average PEP curves show a similar pattern by changing $m$ or $\left(\sigma_{r, d}^{b}\right)^{2}$, i.e., increasing either $\left(\sigma_{r, d}^{b}\right)^{2}$ or $m$ will always enhance end-to-end system performance. It is significant to note that increasing $\left(\sigma_{r, d}^{b}\right)^{2}$ is more significant than increasing $m$ for improvement in system performance.

In Fig. 8, comparisons provide between the system performance for equal and optimal power allocation factors employing 4-PSK modulation symbols. As expected, the PEP for optimal power allocation outperforms PEP for equal power allocation. At high SNR regimes, it has been demonstrated that the simulation results are in close agreement with analytical results derived in (34) and (75) for $\mathrm{DH}$ and $\mathrm{MH}$ relaying protocol, respectively. Further, it is significant to note that for a single relay case, the $\mathrm{DH}$ and 


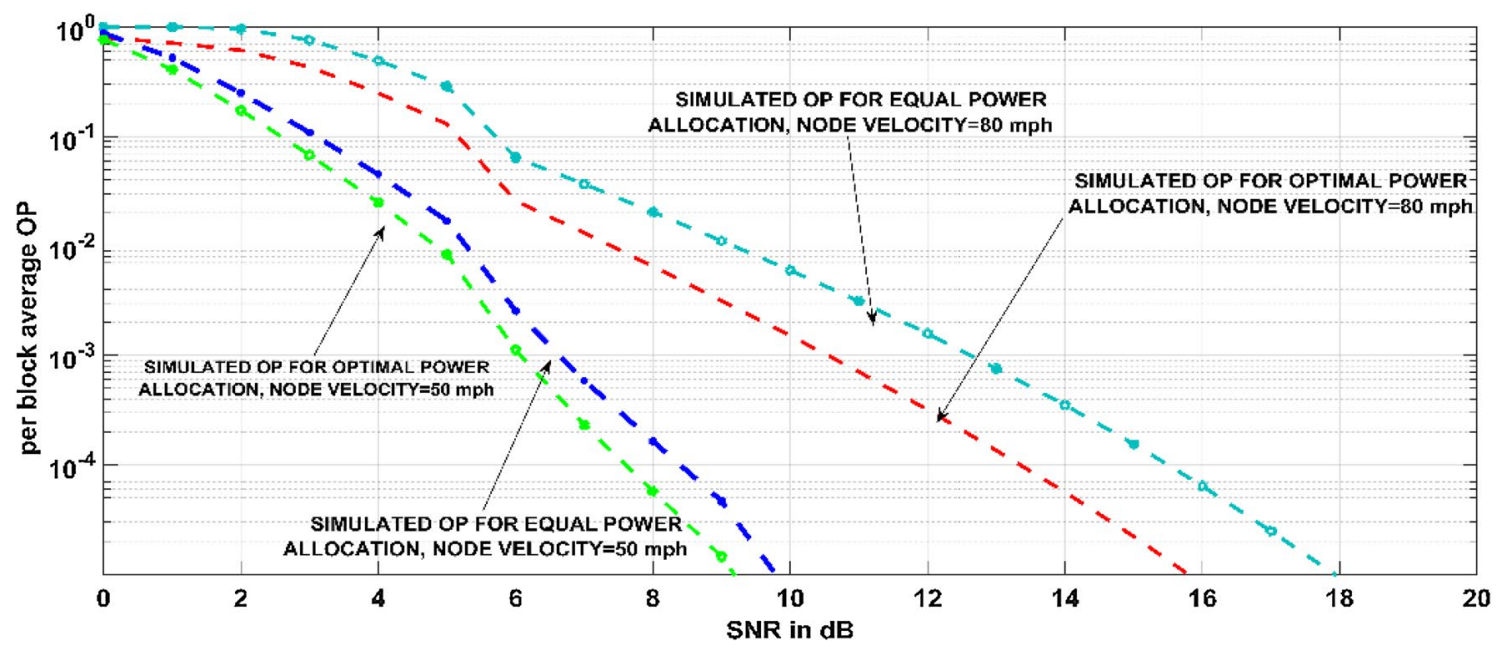

Fig. 17 Per-block average outage probability performance of $\mathrm{DH}$ cooperative communication system for optimal and equal power allocation scenarios for various node velocities with $L=3, m=1, M_{b}=10,\left(\sigma_{s, d}\right)^{2}=\left(\sigma_{s, r}^{b}\right)^{2}=20,\left(\sigma_{r, d}^{b}\right)^{2}=2, \sigma_{e_{i}}^{2}=0.15, \sigma_{\epsilon_{i}}^{2}=0.02$

MH cooperative communication network obtains similar per-block average PEP performance.

In Fig. 9, the per-block average OP performance is demonstrated for a different set of values of distance-dependent PTFs employing 4-QAM modulation symbols. It is significant to observe that the OP decreases with increasing the value of distance-dependent power transfer factors (PTFs). In Fig. 10, it has been demonstrated that with the increase in the node velocity the per-block average PEP performance decreases.

It is significant to note that due to relative velocity between two communication nodes, the channel will become time selective in nature, and in case of timeselective channel with an increase in the channel variance, the end-to-end system performance significantly decreases. Also, for the static channel conditions, i.e., when node velocity is $0 \mathrm{mph}$, system performance is best, and at node velocity $150 \mathrm{mph}$ as shown in Figs. 10 and 11 system performance is the worst. Both the $\mathrm{DH}$ and $\mathrm{MH}$ relaying networks are encounter by error floor limit which arises due to the imperfect channel estimation. The per-block average PEP performance is limited due to this asymptotic error floor limit. For perfect CSI and static nodes, the DO is equal toDO $=\tau_{\mathrm{SD}}+L \min \left(\tau_{\mathrm{SR}}, \tau_{\mathrm{RD}}\right)=18$ for $m=1$ (Rayleigh fading). Furthermore, it is observed that with an increase in the relative velocity between the communicating nodes the channel correlation coefficients for the source-to- $b$ th relay, $b$ th relay-to-destination, and SD node fading links decrease and it is unity for static nodes.

A significant observation is made in case of noncooperative communication that the DO is equal to the $\mathrm{DO}=\tau_{\mathrm{SD}}=9($ for $m=1)$. So if we compared the DO for non-cooperative communication with cooperative communication, we can say that the DO of cooperative communication is very high as compared to the noncooperative communication (18 in case of cooperative communication and 9 in case of non-cooperative communication for Rayleigh channel conditions). Due to better performance, the cooperative communication system is better than the performance of the non-cooperative communication system. In Fig. 12a, b, plots demonstrate the PEP performance of the Alamouti STBC-based $W(1)$ and $W(2)$ relaying network for various values of node velocity. It can be readily seen that with an increase in the relative velocity between two communicating nodes, the PEP performance degrades.

To extend our analysis for non-orthogonal STBC code, $\mathrm{DH}$ system is analyzed with $2 \times 2$ Golden Codes (GC) which is a non-orthogonal code, as shown in Fig. 13. The plots confirm the accuracy of the analytical results, and at high SNR regimes the analytical results are in close agreement with the simulated results. In Fig. 14, the per-block average PEP performance is analyzed for various modulation schemes such as $M$-ary QAM and $M$-ary QPSK. The plots show that 4-PSK performance is good and 8-PSK performance is worst. These outcomes confirm the analytical analysis given in the literature [16-18] that for higher-order constellations the end-to-end performance decreases and it improves for less complex modulation schemes.

In Fig. 15, performance comparison is given for MH S-DF and $\mathrm{MH}$ AF cooperative communication protocol. From the plots, it is observed that the performance of the S-DF relaying protocol is better than the AF relaying protocol. The improvement in performance in case of S-DF protocol is because in case of AF relaying-based network the noise amplification decreases the performance of the system.

In Fig. 16, the per-block average OP is investigated for values of node velocity. For outage probability calculation, 


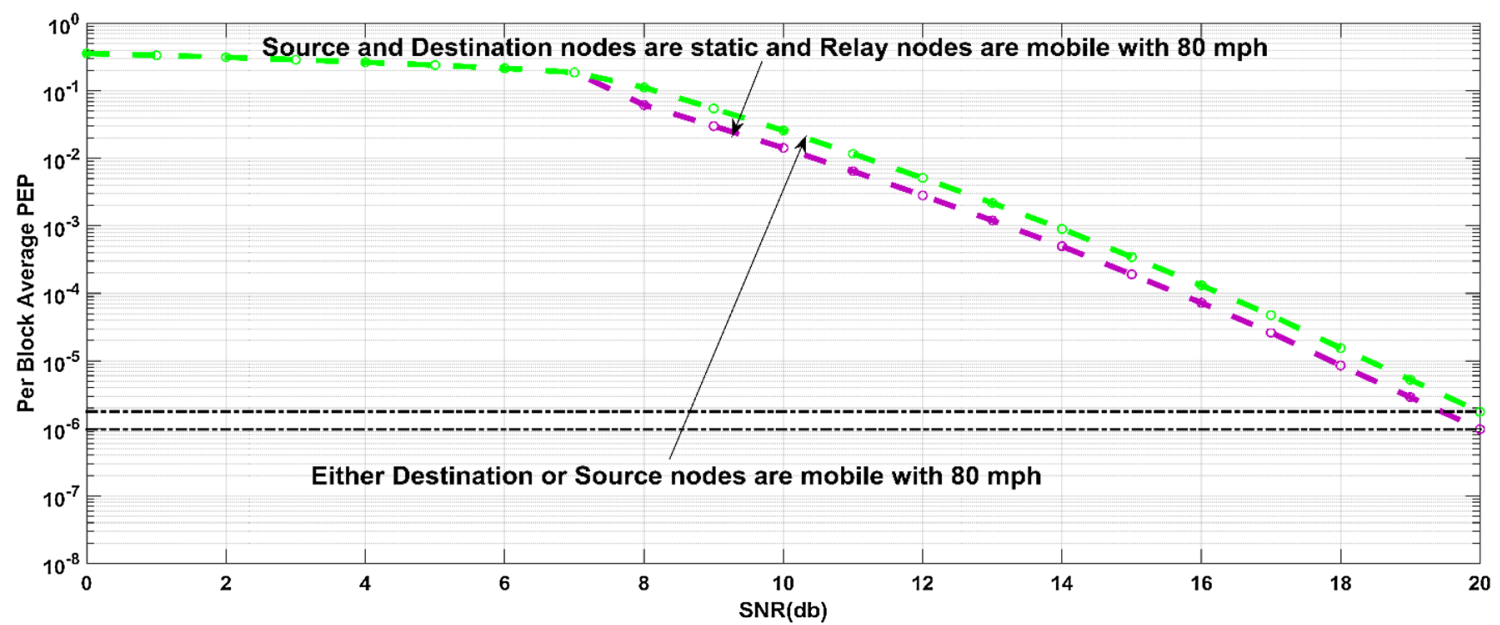

(a)

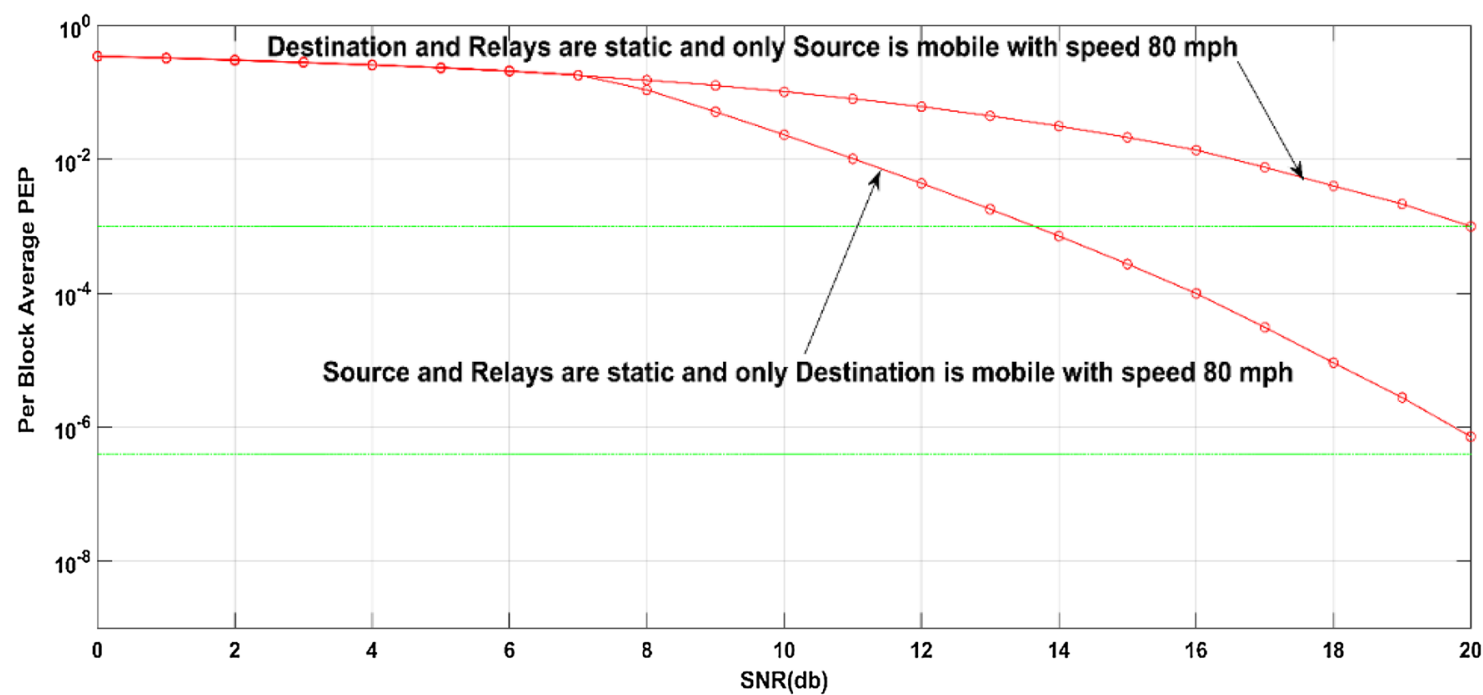

(b)

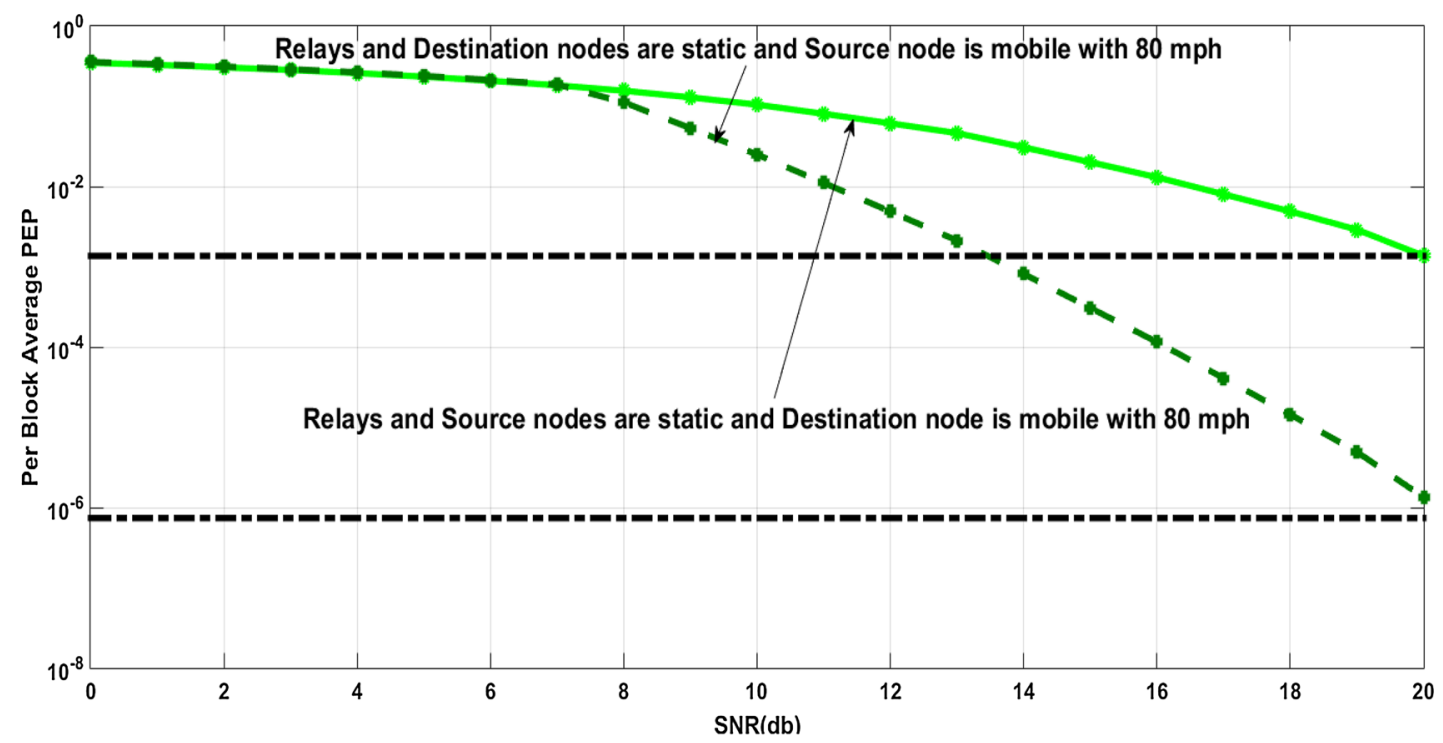

(c) 
4Fig. 18 Per-block average PEP performance of Alamouti STBC based $\mathrm{DH}$ relaying network with $L=3, m=1, M_{b}=15, \sigma_{e}^{2}=0.15,=\sigma_{\epsilon}^{2}=0.02, \quad\left(\sigma_{s, d}\right)^{2}=20$ a $\left(\sigma_{s, r}^{b}\right)^{2}=\left(\sigma_{r, d}^{b}\right)^{2}=2 \forall b, \quad$ b $\quad\left(\sigma_{s, r}^{b}\right)^{2}=2,\left(\sigma_{r, d}^{b}\right)^{2}=20 \forall b, \quad$ c $\left(\sigma_{s, r}^{b}\right)^{2} \stackrel{s, r}{=} 20,\left(\sigma_{r, d}^{b}\right)^{r}=2 \forall b$

the simulator is running freely for number of runs and each run has $10^{6}$ samples. It runs until the statistics are stable and the threshold SNR $\gamma_{0}$ is set as $6 \mathrm{~dB}$. Plots show that theoretical results are in close agreement with the simulated results at high SNR regimes, and with increase in the value of node velocity the OP performance decreases.

In Fig. 17, the per-block average OP is investigated for the optimal and equal power allocation scenarios. In this analysis, it has been observed that the per-block average OP performance for optimal power allocation is better than the OP performance at the equal power allocation. Figure 18a shows that for symmetric channel gains, i.e., $\left(\sigma_{s, r}^{b}\right)^{2}=\left(\sigma_{r, d}^{b}\right)^{2}$, the per-block average PEP performance with static source and destination nodes and mobile relay nodes is better than the scenario when relay and destination nodes are static, and source node is mobile. Moreover, for $\left(\sigma_{s, r}^{b}\right)^{2}=\left(\sigma_{r, d}^{b}\right)^{2}$, both destination and source nodes' mobility, for similar relative velocity, have an identical effect on the per-block average PEP performance. However, for the network conditions when $\left(\sigma_{s, r}^{b}\right)^{2} \leq\left(\sigma_{r, d}^{b}\right)^{2}$ as given in Fig. 18b, source mobility significantly degrades the per-block average PEP performance in contrast to the network condition with only mobile destination node. Moreover, for $\left(\sigma_{s, r}^{b}\right)^{2} \geq\left(\sigma_{r, d}^{b}\right)^{2}$ given in Fig. 18c, mobile destination node significantly degrades the per-block average PEP performance in contrast to the network condition with only mobile source node. Further, one can readily see from Fig. 19 that when source and destination nodes are static and mobile relay nodes with perfect CSI knowledge at each receiving terminal, the per-block average PEP performance is not limited by an error floor limit and DO of $\tau_{\mathrm{SD}}=4$ is achieved.

In Fig. 20a, b, per-block average PEP is analyzed for both $\mathrm{DH}$ and $\mathrm{MH}$ protocols for optimal power allocation factors $\beta_{0}, \beta_{b}, 1 \leq b \leq L$. The optimal power factors are obtained by solving the optimization problem by using the CVX software. The PEP performance for optimal power notably enhances the per-block PEP performance in contrast to the scenario for different channel conditions. Also, system performance improves significantly when $\left(\sigma_{s, r}^{b}\right)^{2} \leq\left(\sigma_{r, d}^{b}\right)^{2}$ as demonstrated in Fig. 20a, b.

\section{Conclusion}

In this paper, we investigate the S-DF cooperative communication system over time-selective Nakagami- $m$ fading links in the presence of nodes' mobility and channel estimation errors. To evaluate the PEP and outage performance of S-DF-based relaying network, the CF expressions of OP and per-block average PEP are derived carefully, considering constraints at the relay, source, and destination nodes.

It is observed that with an increase in the relative velocity between the communicating nodes, the channel correlation coefficients for the source-to-bth relay, $b$ th relayto-destination, and SD node fading links decrease and it is unitary for static nodes. Both the $\mathrm{DH}$ and the $\mathrm{MH}$ relaying networks are encountered by error floor limit which arises due to the imperfect channel estimation. Further, for perfect CSI and static nodes, the DO is equal to 18 for Rayleigh fading. Furthermore, a significant observation is made in case of direct communication (non-cooperative) that the DO is equal to 9 for Rayleigh fading.

Due to better performance, the cooperative communication system is better than the performance of the noncooperative communication system. Simulation results demonstrate that the due to time-selective nature of the fading links, the per-block average PEP performance is degraded and achieves the asymptotic error floor limit at high SNR regimes. Results show that increasing shape parameter and RD channel gain always enhances endto-end system performance. It is significant to note that increasing $\mathrm{RD}$ is more significant than increasing shape parameter for improvement in system performance.

Also, it can be readily seen that the increasing the RD link gain is more significant than increasing the sourceto-destination or source-to-relay fading link gain. As expected, the PEP for optimal power allocation outperforms PEP for equal power allocation. It is demonstrated that the OP decreases with increasing the value of distance-dependent PTFs. The plots show the PEP performance of the Alamouti STBC-based $W(1)$ and $W(2)$ relaying network for various values of node velocity. It can be readily seen that with an increase in the relative velocity between two communicating nodes, the PEP performance degrades.

Further, it has been observed that due to the mobility of nodes and imperfect CSI, the PEP performance of the system experiences degradation especially for high values of SNR. Furthermore, when all the nodes are static and 


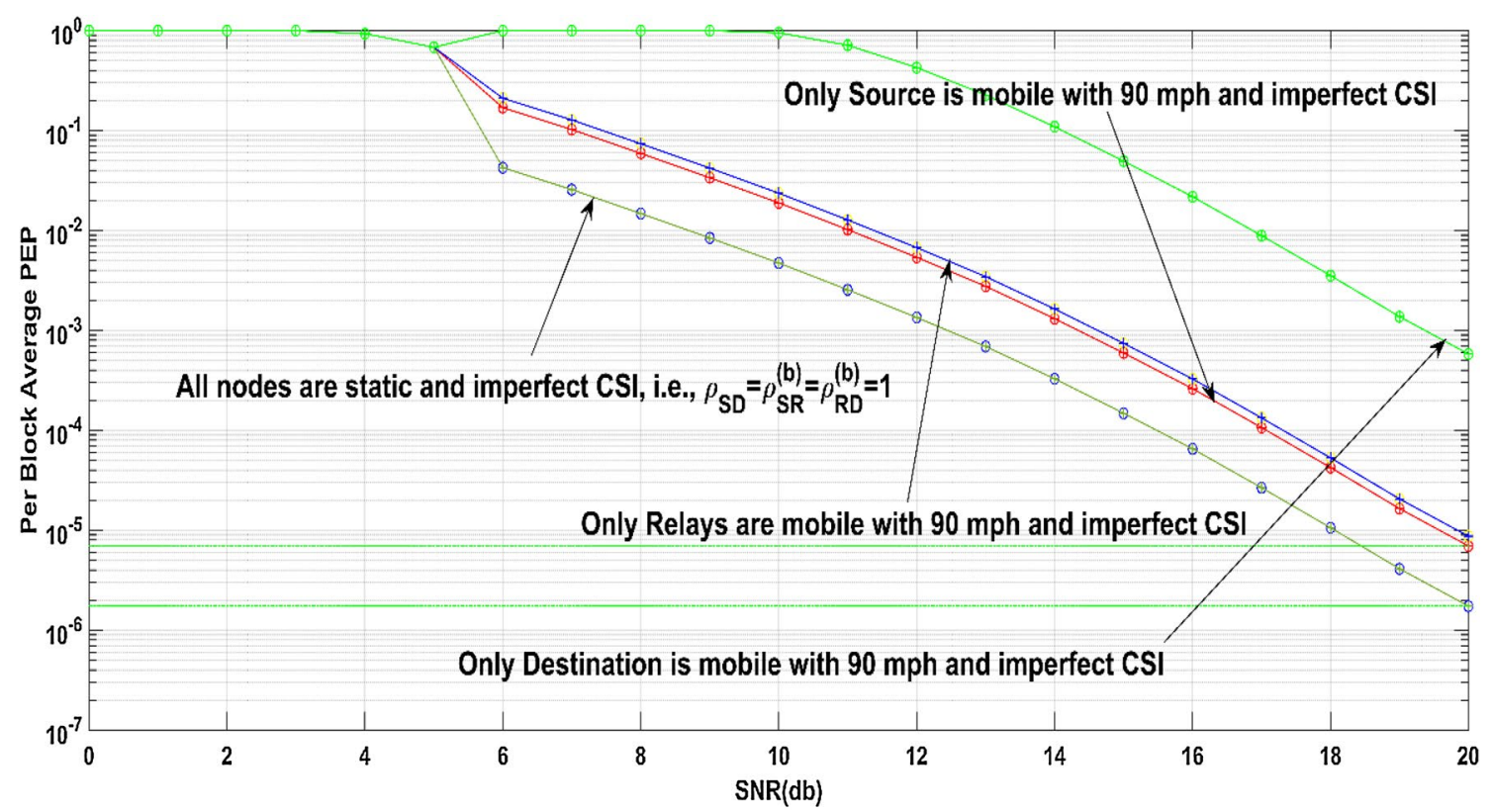

(a)

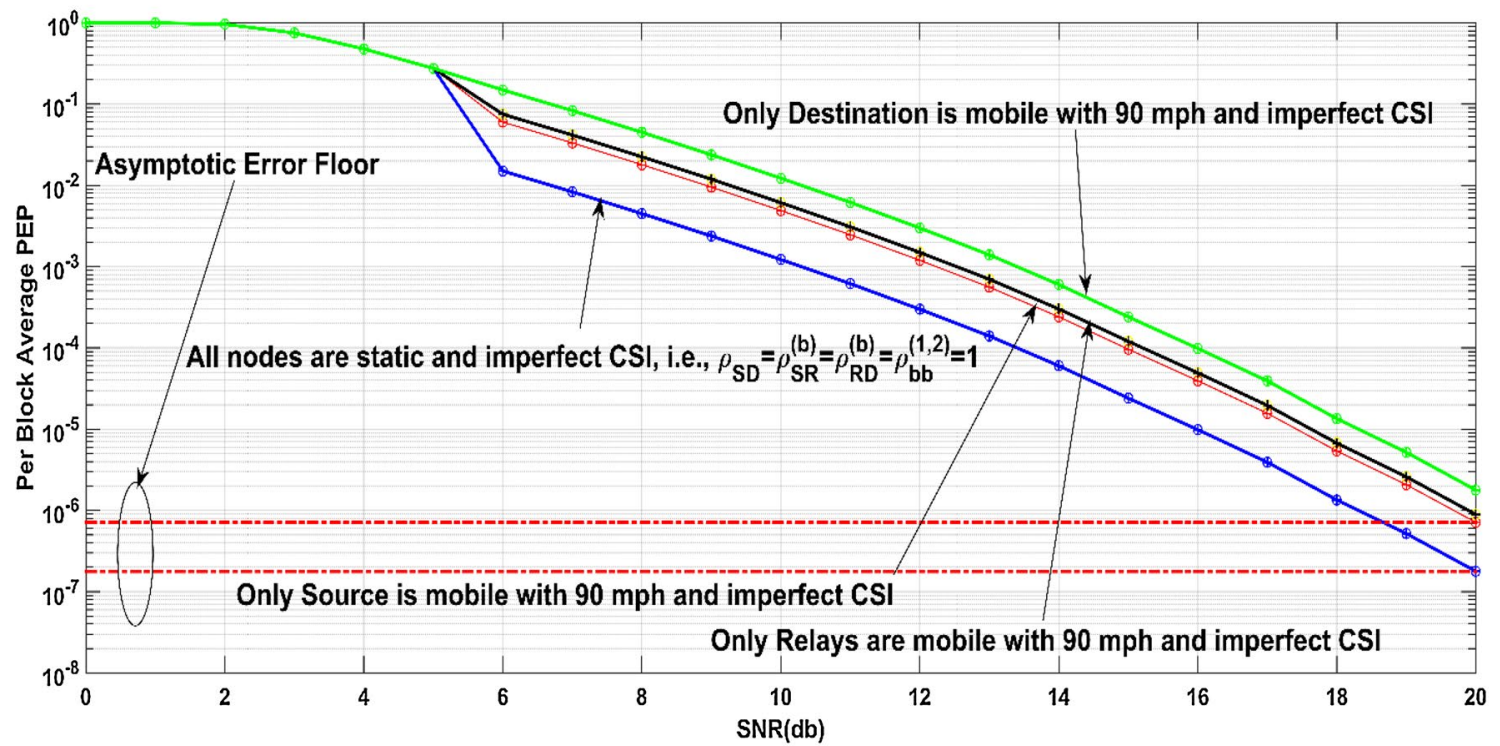

(b)

Fig. 19 Per-block average PEP performance of Alamouti STBC a DH and b MH W(1) relaying network with $L=3, m=1, M_{b}=10,\left(\sigma_{s, d}\right)^{2}=\left(\sigma_{s, r}^{b}\right)^{2}=\left(\sigma^{b, \tilde{b}}\right)^{2}=20,\left(\sigma_{r, d}^{b}\right)^{2}=2, \sigma_{e_{i}}^{2}=0.15, \sigma_{\epsilon_{i}}^{2}=0.02$. 


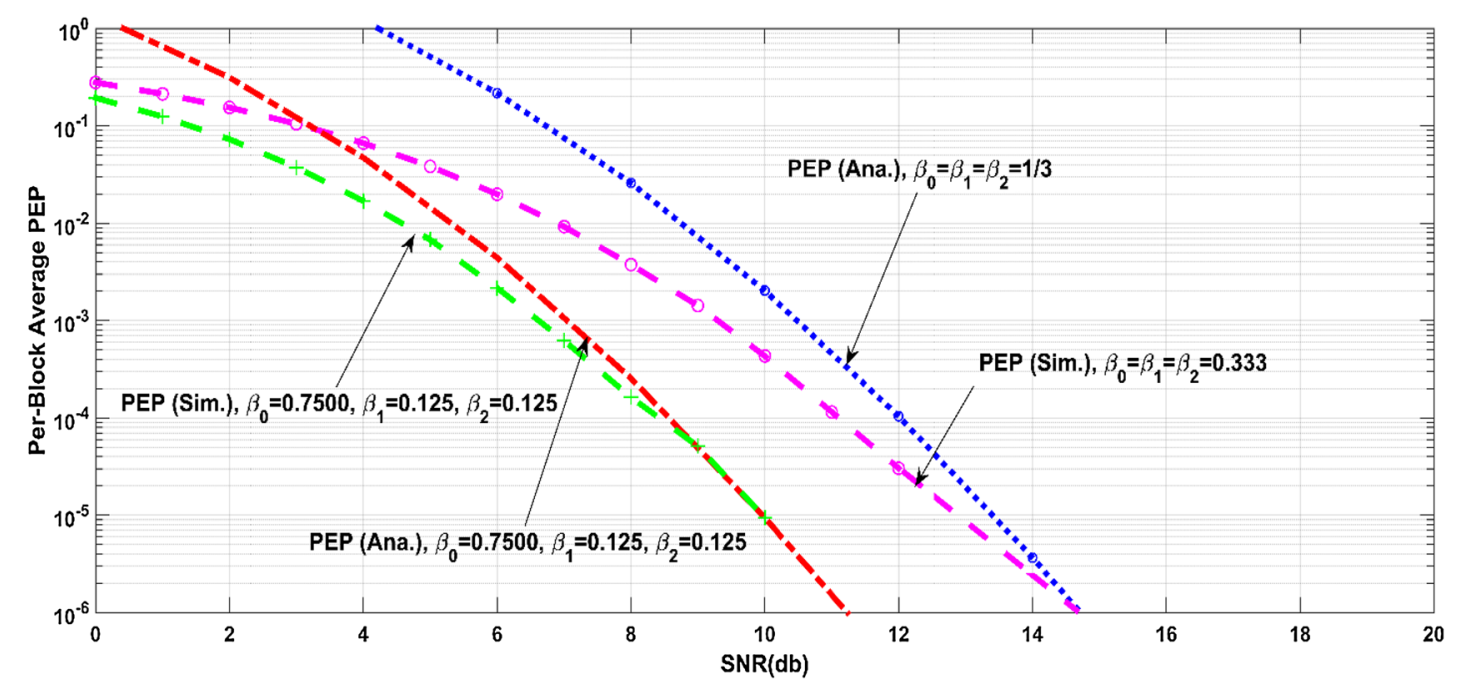

(a)

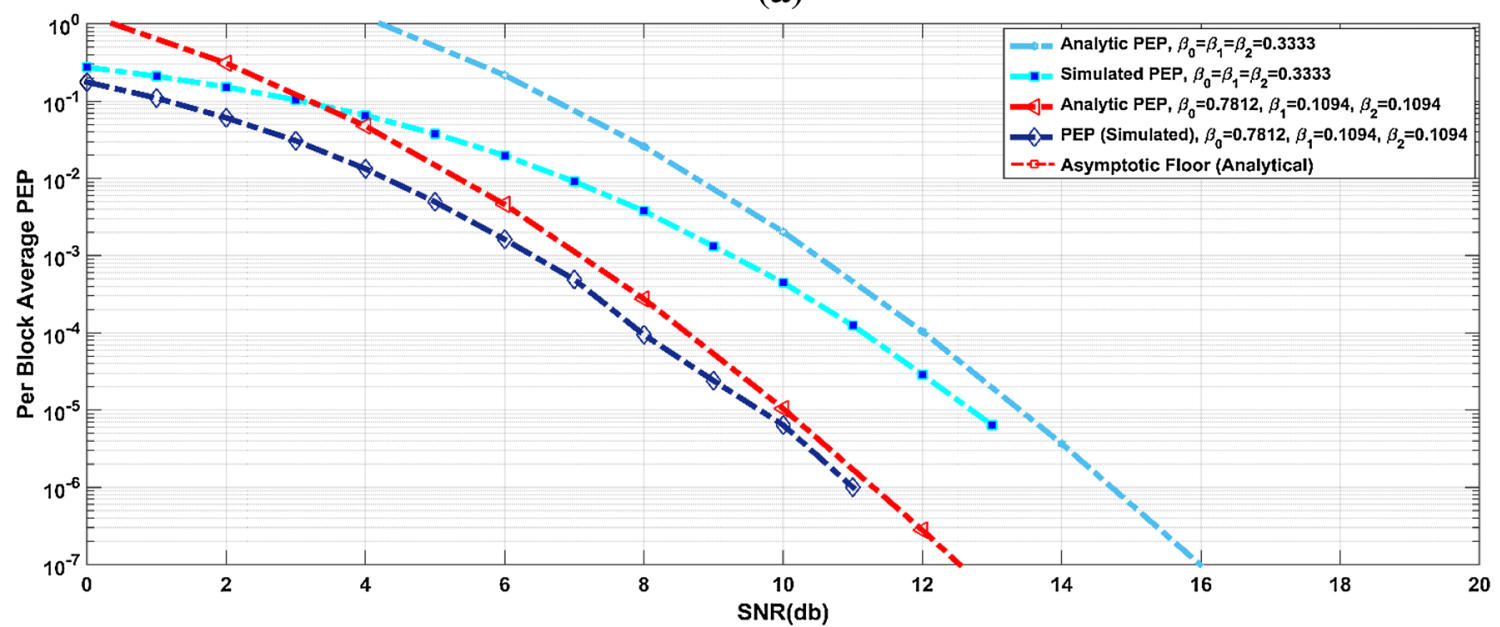

(b)

Fig. 20 Per-block average PEP performance of MIMO STBC relaying network employing a $\mathrm{DH}$ protocol with $\beta_{0}=0.750, \beta_{1}=0.125, \beta_{2}=0.125, M_{b}=15, \sigma_{e_{i}}^{2}=0.15, \sigma_{\epsilon_{i}}^{2}=0.02, m=1$

b $W(1)$ protocol with optimal power factors

$\beta_{0}=0.7812, \beta_{1}=0.1094, \beta_{2}=0.1094, M_{b}=15, \sigma_{e_{i}}^{2}=0.15, \sigma_{\epsilon_{i}}^{2}=0.02, \sigma_{e b \tilde{b}}^{2}=0.15, \sigma_{\in b \tilde{b}}^{2}=0.02, m=1$

the estimation processes are perfect, the asymptotic error floors vanish, i.e., the nodes' mobility impact is removed. It has been observed that the S-DF relaying network outperforms the AF relaying network over time-selective fading links. At high SNR regimes, it has been demonstrated that the simulation results are in close agreement with analytical results derived for $\mathrm{DH}$ and $\mathrm{MH}$ relaying protocol, respectively.

\section{Compliance with ethical standards}

Conflict of interest The authors declare that they have no conflict of interest.

\section{References}

1. Harri H, Toskala A, Nakamura T (2020) 5 G technology: 3GPP new radio. Wiley, Hoboken. ISBN 978-1-119-23629-0

2. Dahlman E, Parkvall S, Skold J (2014) 4G: LTE/LTE-advanced for mobile broadband, 2nd edn. Academic Press, Cambridge. https ://doi.org/10.1016/C2013-0-06829-6

3. Shankar R, Bhardwaj L, Mishra RK (2020) Analysis of selectivedecode and forward relaying protocol over $\mathrm{k}-\mu$ fading channel 
distribution. J Telecommun Inf Technol 1:21-30. https://doi. org/10.26636/jtit.2020.135919

4. Shankar R, Mishra RK (2019) S-DF cooperative communication system over time selective fading channel. J Inf Sci Eng 35(6):1223-1248. https://doi.org/10.6688/JISE.20191 1_35(6).0004

5. Sheng AZ, Liu CH (2016) Energy efficient cooperative wireless communication and networks. CRC Press, Boca Raton

6. I Krikidis, G Zheng (2016) Advanced relay technologies in next generation wireless communications. Institution of Engineering and Technology. ISBN: 978-1-78561-003-5

7. Asshad M, Khan SA, Kavak A, Küçük K, Msongaleli DL (2019) Cooperative communications using relay nodes for next-generation wireless networks with optimal selection techniques: a review. IEEJ Trans Electr Electron Eng. https://doi.org/10.1002/ tee. 22852

8. Zhang X, Kang S, Fu X (2020) Pattern division multiple access featuring amplify-and-forward relaying in an uplink network. IEEE Access 8:85656-85663. https://doi.org/10.1109/ACCES S.2020.2992455

9. Jeong Ui-Seok, Ryu Gi-Hoon, Jang Dong-Sun, Ko Kyunbyoung (2019) Exact SEP and performance bounds for orthogonal space-time block codes in cooperative multiantenna AF relaying networks. Int J Commun Syst 32(9):e3943. https://doi. org/10.1002/dac.3943

10. Ali B, Mirza J, Zhang J, Zheng G, Saleem S, Wong K (2019) Fullduplex amplify-and-forward relay selection in cooperative cognitive radio networks. IEEE Trans Veh Technol 68(6):6142-6146. https://doi.org/10.1109/TVT.2019.2907468

11. Lu Y, Mow WH (2019) Low-complexity detection and performance analysis for decode-and-forward relay networks. In: IEEE international conference on acoustics, speech and signal processing (ICASSP), ICASSP 2019, Brighton, United Kingdom, pp 4819-4823. https://doi.org/10.1109/ICASSP.2019.8682497

12. Sahin Y, Erdogan E, Gucluoglu T (2019) Performance analysis of two way decode and forward relay network with maximum ratio transmission. Phys Commun 32:75-80. https://doi.org/10.1016/j. phycom.2018.11.004

13. Shankar R, Mishra RK (2018) An investigation of S-DF cooperative communication protocol over keyhole fading channel. Phys Commun 29:120-140. https://doi.org/10.1016/j.phyco m.2018.04.027

14. Shankar R, Kumar I, Mishra RK (2019) Outage probability analysis of MIMO-OSTBC relaying network over Nakagami-m fading channel conditions. Traitement du Signal 36(1):59-64. https:// doi.org/10.18280/ts.360108

15. Khattabi YM, Matalgah MM (2016) Performance analysis of multiple-relay af cooperative systems over Rayleigh timeselective fading channels with imperfect channel estimation. IEEE Trans Veh Technol 65(1):427-434. https://doi.org/10.1109/ TVT.2015.2399295

16. Khattabi Y, Matalgah MM (2015) Conventional AF cooperative protocol under nodes-mobility and imperfect-CSI impacts: outage probability and Shannon capacity. In: IEEE wireless communications and networking conference, New Orleans, LA, pp 13-18. https://doi.org/10.1109/WCNC.2015.7127437

17. Khattabi YM, Matalgah MM (2018) Alamouti-OSTBC wireless cooperative networks with mobile nodes and imperfect CSI estimation. IEEE Trans Veh Technol 67(4):3447-3456. https:// doi.org/10.1109/TVT.2017.2786471

18. Kumar I, Sachan V, Shankar R, Mishra RK (2018) An investigation of wireless S-DF hybrid satellite terrestrial relaying network over time selective fading channel. Traitement du Signal 35(2):103120. https://doi.org/10.3166/TS.35.103-120
19. Varshney N, Jagannatham AK (2016) Performance analysis of MIMO-OSTBC based selective DF cooperative wireless system with node mobility and channel estimation errors. In: Twenty second national conference on communication (NCC), Guwahati, pp 1-6. https://doi.org/10.1109/NCC.2016.7561110

20. Shankar R, Kumar G, Sachan V, Mishra RK (2018) An investigation of two phase multi-relay S-DF cooperative wireless network over time-variant fading channels with incorrect CSI. Procedia Comput Sci 125:871-879. https://doi.org/10.1016/j.procs .2017.12.111

21. Sahu HK, Sahu PR (2019) Use of Nakagami-m fading channel in SSK modulation and its performance analysis. Wirel Pers Commun 108(2):1261-1273

22. Beaulieu NC, Cheng C (2005) Efficient Nakagami-m fading channel simulation. IEEE Trans Veh Technol 54(2):413-424

23. Yip K-W, Ng T-S (2000) A simulation model for Nakagami-m fading channels, $m<1$. IEEE Trans Commun 48(2):214-221

24. Airod, FE, Chafnaji $\mathrm{H}$, Tamtaoui A (2017) Performance analysis for amplify and forward full-duplex relaying with direct link over Nakagami-m fading channel. In: International conference on wireless networks and mobile communications (WINCOM), Rabat, pp 1-6. https://doi.org/10.1109/WINCOM.2017.8238163

25. Nguyen BC, Hoang TM, Tran PT (2019) Performance analysis of full-duplex decode-and-forward relay system with energy harvesting over Nakagami-m fading channels. AEU Int J Electron Commun 98:114-122

26. Pandey A, Yadav S (2019) Physical layer security in cooperative amplify-and-forward relay networks over mixed Nakagami-m and double Nakagami-m fading channels: performance evaluation and optimisation. IET Commun 14(1):95-104

27. Datta R, Gurjar DS, Manohar Reddy TK, Chaupal SK, Mandloi M, Hossain A (2020) Secrecy performance of amplify-and-forward relay networks with relay selection under Nakagami-m fading. Wirel Pers Commun 112:2233-2251

28. Nair G, Kappla S (2020) Machine-to-machine network optimization and online charging. U.S. Patent Application 16/567,378. Affirmed Networks Inc

29. Chun YJ, Cotton SL, Dhillon HS, Ghrayeb A, Hasna MO (2017) A stochastic geometric analysis of device-to-device communications operating over generalized fading channels. IEEE Trans Wirel Commun 16(7):4151-4165

30. Varshney N, Jagannatham AK (2017) MIMO-STBC based multiple relay cooperative communication over time-selective Rayleigh fading links with imperfect channel estimates. IEEE Trans Veh Technol 66(7):6009-6025. https://doi.org/10.1109/ TVT.2016.2634924

31. Baddour KE, Beaulieu NC (2005) Autoregressive modeling for fading channel simulation. IEEE Trans Wirel Commun 4(4):16501662. https://doi.org/10.1109/TWC.2005.850327

32. Zhou Y, Cheng N, Lu N, Shen XS (2015) Multi-UAV-aided networks: aerial-ground cooperative vehicular networking architecture. IEEE Veh Technol Mag 10(4):36-44

33. Zhou Z, Houjian Yu, Chen X, Zhang Y, Mumtaz S, Rodriguez J (2018) Dependable content distribution in D2D-based cooperative vehicular networks: a big data-integrated coalition game approach. IEEE Trans Intell Transp Syst 19(3):953-964

34. Soatti G, Nicoli M, Garcia N, Denis B, Raulefs R, Wymeersch H (2018) Implicit cooperative positioning in vehicular networks. IEEE Trans Intell Transp Syst 19(12):3964-3980

35. Bazzi A, Campolo C, Masini BM, Molinaro A, Zanella A, Berthet AO (2018) Enhancing cooperative driving in IEEE 802.11 
vehicular networks through full-duplex radios. IEEE Trans Wirel Commun 17(4):2402-2416

36. Asif HM (2020) Analysis of space time block codes and cosets for 5G and its applications. Comput Commun 149:189-193

37. Kalbasi K, Jamaloddin Golestani S (2020) On the relaxed maximum-likelihood blind MIMO channel estimation for orthogonal space-time block codes. Signal Process 175:107653

38. Liu KJR, Sadek AK, Su W, Kwasinski A (2009) Cooperative communications and networking. Cambridge University Press, Cambridge

39. Varshney N, Krishna AV, Jagannatham AK (2015) Selective DF protocol for MIMO STBC based single/multiple relay cooperative communication: end-to-end performance and optimal power allocation. IEEE Trans Commun 63(7):2458-2474

40. Khattabi Y, Matalgah MM (2015) Conventional and best-relayselection cooperative protocols under nodes-mobility and imperfect-CSI impacts: BER performance. In: IEEE wireless communications and networking conference (WCNC). IEEE, pp 105-110

41. Varshney N, Jagannatham AK, Varshney PK (2018) Cognitive MIMO-RF/FSO cooperative relay communication with mobile nodes and imperfect channel state information. IEEE Trans Cognit Commun Netw 4(3):544-555

42. Varshney N, Puri P (2017) Performance analysis of decodeand-forward-based mixed MIMO-RF/FSO cooperative systems with source mobility and imperfect CSI. J Lightwave Technol 35(11):2070-2077

43. Simon MK, Alouini M-S (2005) Digital communication over fading channels, vol 95. Wiley, Hoboken

44. IIlhan $\mathrm{H}$, Altunbaş İ, Uysal $\mathrm{M}$ (2011) Moment generating functionbased performance evaluation of amplify-and-forward relaying in $\mathrm{N}^{*}$ Nakagami fading channels. IET Commun 5(3):253-263

45. Aly RM, Zaki A, Badawi WK, Aly MH (2019) Time coding OTDM MIMO system based on singular value decomposition for $5 \mathrm{G}$ applications. Appl Sci. https://doi.org/10.3390/app9132691
46. Gradshteyn IS, Ryzhik IM (2014) Table of integrals, series, and products. Academic Press, New York

47. Lofberg J (2004) YALMIP: a toolbox for modeling and optimization in MATLAB. In: IEEE international conference on robotics and automation (IEEE Cat. No. 04CH37508), pp 284-289

48. Varshney N, Krishna AV, Jagannatham AK (2014) Capacity analysis for path selection-based DF MIMO-OSTBC cooperative wireless systems. IEEE Commun Lett 18(11):1971-1974

49. Balti E, Guizani M (2018) Mixed RF/FSO cooperative relaying systems with co-channel interference. IEEE Trans Commun 66(9):4014-4027

50. Gradshteyn IS, Ryzhik IM (2000) Table of integrals, series, and products, 6 th edn. Academic Press, New York

51. Nesterov $Y$ (2013) Introductory lectures on convex optimization: a basic course, vol 87. Springer, Berlin

52. Dattorro J (2010) Convex optimization and Euclidean distance geometry. Lulu.com

53. Grant M, Boyd S (2014) CVX: Matlab software for disciplined convex programming, version 2.1

54. Heimonen A, Matala-Aho T, Väänänen K (1993) On irrationality measures of the values of Gauss hypergeometric function. Manuscr Math 81(1):183-202

55. Gordon G, Tibshirani R (2012) Karush-Kuhn-Tucker conditions. Optimization 10(72536):725

Publisher's Note Springer Nature remains neutral with regard to jurisdictional claims in published maps and institutional affiliations. 